\title{
Qualitätszielfunktionen für stark variierende Gemeindegrößen im Zensus 2021
}

\author{
Jan Pablo Burgard · Ralf Münnich $($ D $\cdot$ Martin Rupp
}

Eingegangen: 8. März 2019 / Angenommen: 30. August 2019 / Online publiziert: 17. September 2019 (C) Der/die Autor(en) 2019

Zusammenfassung Am 24. Oktober 2017 wurden beim Bundesverfassungsgericht die Normenkontrollklagen der Länder Berlin und Hamburg im Bezug auf das Zensusgesetz 2011 verhandelt. Ein zentraler Gegenstand der Klagen war eine methodische Ungleichbehandlung von Gemeinden unterschiedlicher Größe bei der Einwohnerzahlermittlung im Zensus 2011, da für Gemeinden kleiner als 10.000 Einwohner eine andere Methodik angewendet wurde als für Gemeinden mit mehr als 10.000 Einwohnern. Diese Grenze wurde im Zensusgesetz für den Zensus 2011 festgeschrieben und ist nach Bekanntwerden der Zensusergebnisse kritisiert worden. Im vorliegenden Beitrag werden verschiedene Möglichkeiten diskutiert, wie beim Zensus 2021 auf einen Methodenwechsel, wie er im Jahr 2011 stattfand, verzichtet werden kann und wie dahingehend die Methodik geeignet angepasst werden muss. Einen besonderen Schwerpunkt nimmt hierbei eine Adjustierung der Qualitätsmessung, die sogenannten Präzisionsvorgaben, ein. Eine Definition dieser für

\footnotetext{
Diesen Artikel widmen wir einer großartigen Freundin, Wissenschaftlerin und Kollegin, Frau Professor Dr. Susanne Rässler, die leider viel zu früh von uns gegangen ist (https://www. uni-bamberg.de/stat-oek/nachruf/). Sie beeindruckte stets mit ihrer offenen, freundlichen und einnehmenden Art. Als unabhängige Gutachterin beim Bundesverfassungsgericht war sie sehr eng mit der Thematik dieses Artikels verbunden. Sie hat sich mit uns sehr intensiv und positiv über die vorläufigen Forschungsarbeiten ausgetauscht. Zudem hat sie schon sehr auf die Diskussion dieses Artikels gewartet. Dies, wie auch die Verlesung des Urteils in Karlsruhe war ihr leider nicht mehr vergönnt. Susanne, wir vermissen Dich!
}

J. P. Burgard · R. Münnich $(\bowtie) \cdot$ M. Rupp

FB IV, VWL, Wirtschafts- und Sozialstatistik, Universität Trier, Universitätsring 15, 54296 Trier, Deutschland

E-Mail: muennich@uni-trier.de

J. P. Burgard

E-Mail: burgardj@uni-trier.de

M. Rupp

E-Mail: ruppm@uni-trier.de 
eine stichprobenbasierte Zensuserhebung muss insbesondere im Hinblick auf die Gemeindegröße geeignet gewählt werden. Zudem bedarf es einer Untersuchung der Auswirkung der Qualitätsmessung auf die regionalen Stichprobenumfänge und auf die Qualität der später zu schätzenden Einwohnerzahl. In den Untersuchungen werden methodische und praktische Überlegungen berücksichtigt.

Schlüsselwörter Zensus · Ermittlung der Einwohnerzahl · Qualitätsmessung · Optimale Allokation · Box-Constraints

\title{
Quality measures respecting highly varying community sizes within the 2021 German Census
}

\begin{abstract}
On 24 October 2017, the federal states Berlin and Hamburg have filed a lawsuit against the law of the 2011 German Census at the Federal Constitutional Court of Germany. A major subject was the unequal methodological treatment of communities of different sizes in the determination of the population size in the 2011 German Census, in which municipalities above a threshold of 10,000 inhabitants were treated according to a different method than communities below this threshold. The limit of 10,000 inhabitants was defined in the Census Act for the 2011 German Census and was criticised after the publication of the census results. In this article we discuss various options for avoiding this change of method in the 2021 German Census. In this regard, a suitable adaption of the methods utilised in 2011 is presented. The particular focus is on adjusting the quality measurement which is defined by specific precision targets. A definition of these targets for a register-assisted census has to be chosen appropriately considering especially the sizes of communities. Further, the precision targets have to be examined with regard to their effects on the regional sample sizes and on the quality of the population size estimates. Several methodological and practical considerations will be taken into account within this study.
\end{abstract}

Keywords Census - Determination of Population Size - Quality Measurement · Optimal Allocation · Box-Constraints

\section{Einleitung und Motivation}

Im Jahr 2011 fand in Deutschland der erste registergestützte Zensus statt (siehe Zensusgesetz 2011). Zur Ermittlung der amtlichen Einwohnerzahl wurden Informationen aus den Melderegistern sowie aus einer zusätzlich erhobenen Stichprobe verwendet. Darüber hinaus wurde eine Vollerhebung in Sonderbereichen (Gemeinschaftsunterkünfte und Wohnheime) durchgeführt, auf die nachfolgend nicht weiter eingegangen wird, da sie für die in diesem Artikel behandelte Stichprobenmethodik irrelevant ist. Die Zensusstichprobe diente zwei Zielen. Im Vordergrund stand die Schätzung von Über- und Untererfassungen im Melderegister zur Ermittlung der amtlichen Einwohnerzahl. Diese Über- und Untererfassungen werden im Folgenden mit Karteileichen und Fehlbeständen bezeichnet. Darüber hinaus wurden zahlreiche weitere 
Variablen mit Hilfe der Informationen aus der Stichprobe geschätzt. Dabei dienten Anschriften als Stichprobeneinheiten, um eine Aufdeckung von Karteileichen und Fehlbeständen zu ermöglichen. Als Rahmen für die Ziehung wurde das sogenannte Anschriften- und Gebäuderegister verwendet. Eine eingehende Beschreibung der Vorgehensweise im Zensus 2011 findet sich unter http://www.zensus2011.de sowie in Statistische Ämter des Bundes und der Länder (2015). Die Stichprobenmethoden sowie die Schätzmethodik wurden im Zensus-Stichprobenforschungsprojekt erforscht. Die Ergebnisse sind in Münnich et al. 2012a dargestellt. Zum Zeitpunkt der Untersuchungen im Stichprobenforschungsprojekt standen die Ergebnisse des Zensustests, einer vorgeschalteten Untersuchung, zur Verfügung (siehe auch Statistische Ämter des Bundes und der Länder 2004). Ebenso konnte ein anonymisierter Abzug der Melderegister verwendet werden, welcher eine bedeutende Rolle für die auf Simulationen basierenden Untersuchungen spielte.

Die Bekanntmachung der Ergebnisse des Zensus 2011 führte zu einigen Diskussionen. Zunächst wurde in einigen Gegenden, insbesondere in Baden-Württemberg, eine ungewöhnlich hohe Häufigkeit an Nullanschriften festgestellt. Unter Nullanschriften versteht man Anschriften, welche im Ziehungsrahmen enthalten sind, in denen sich jedoch keine gemeldete Person befindet. Weisen diese als Nullanschriften deklarierten Anschriften Wohnraum auf, so sollten sie korrekterweise im Ziehungsrahmen enthalten sein. Da für die Analysen im Rahmen des Stichprobenforschungsprojektes ausschließlich anonymisierte Melderegister als Ziehungsrahmen verwendet wurden, war die Analyse der Auswirkungen von Nullanschriften nicht Teil des Projektes. Um den Einfluss der unerwartet hohen Anzahl an Nullanschriften auf die Empfehlungen des Stichprobenforschungsprojektes zu analysieren, wurde ein Validierungsprojekt durchgeführt, dessen Ergebnisse in Münnich et al. (2013a) dargestellt sind. Es wurde ermittelt, dass sich die Qualität der einzelnen Schätzungen auf Grund der großen Anzahl an Nullanschriften zwar reduziert wurde, die methodischen Empfehlungen jedoch unverändert erhalten bleiben können.

Zwei weitere Auffälligkeiten in den Ergebnissen wurden öffentlich in Frage gestellt. In der Presse wurden insbesondere Zensusergebnisse auf sehr kleinen Nachweiseinheiten diskutiert. Besonders bekannt ist die Diskussion über die geschätzte Geschlechterproportion der Ein- bis Siebenjährigen in Flensburg. ${ }^{1}$ Das generelle Ziel des Verfahrens im Zensus war jedoch der Ausweis der Gesamtpopulation in Gemeinden ab 10.000 Einwohner mit einer im Zensusgesetz festgelegten (a-priori-) Genauigkeit. Die darauf optimierte Verfahrensweise ist nicht ohne weiteres auf beliebig kleine Teilgesamtheiten von deutlich weniger als 10.000 Einwohnern übertragbar, ${ }^{2}$ sodass Qualitätsanforderungen an Geschlechterproportionen auf sehr kleinen Nachweiseinheiten nicht in der Methodik berücksichtigt werden konnten.

Ein weiterer zentraler Diskussionspunkt war die Frage des Methodenwechsels zwischen Gemeinden mit weniger und mehr als 10.000 Einwohnern. Im Zensus-

\footnotetext{
1 siehe https://www.shz.de/lokales/flensburger-tageblatt/flensburg-klagt-gegen- den-zensus-id12531031. html

${ }^{2}$ Hierbei sei angemerkt, dass es sich um eine bekannte Eigenschaft des eingesetzten GREG-Schätzers handelt. Um trotzdem auf kleinen Nachweiseinheiten stabile und kohärente Schätzungen im Zensus 2021 zu gewährleisten, haben Burgard et al. (2019) ein generalisiertes Kalibrierungsverfahren entwickelt.
} 
gesetz wurde für die Stichprobenerhebung eine vordefinierte Mindestqualität für Nachweiseinheiten ab einer Größe von 10.000 Einwohnern festgelegt. Im Gegensatz dazu fand in Gemeinden mit weniger als 10.000 Einwohnern eine Befragung zur Klärung von Unstimmigkeiten (BKU) zur Qualitätssicherung der Einwohnerzahlen statt (vergleiche Statistische Ämter des Bundes und der Länder 2015). Dieses abweichende Vorgehen begründete sich durch eine erhebliche Kostenersparnis in diesen Gebieten. Nach Veröffentlichung konnten allerdings auffällige Diskrepanzen in den Zensusergebnissen über und unter dieser Einwohnergrenze festgestellt werden. Christensen et al. (2015) zeigen auf, dass Gemeinden unterhalb von 10.000 Einwohnern im Vergleich zu Gemeinden mit mehr als 10.000 Einwohnern signifikant geringere relative Verluste in den Einwohnerzahlen aufwiesen. Aus diesen Abweichungen ist zu schließen, dass die ursprüngliche Vorgehensweise einer eingehenden Untersuchung bedarf, bevor sie in weitestgehend analoger Form für den folgenden Zensus 2021 Anwendung finden kann. Darüber hinaus wird in Rendtel (2015) dargelegt, dass der Umfang dieser Abweichungen in Bundesländern mit zentral geführten Registern erheblich geringer ist als in anderen. Da im Rahmen des Datenschutzes für den Zensus 2011 die Hilfsmerkmale früh gelöscht werden mussten und in Gemeinden mit weniger als 10.000 Einwohnern ein Korrekturverfahren an Stelle einer stichprobenbasierten Schätzung verwendet wurde, lässt sich final keine präzise Analyse zur Ursachenklärung durchführen.

Im Jahr 2015 reichten die Länder Berlin und Hamburg eine Normenkontrollklage beim Bundesverfassungsgericht ein (siehe Göß1 2018). Ein Gegenstand war eine Überprüfung der Methodik und insbesondere ein vermeintlicher Versto $\beta$ gegen das Gebot interföderaler und interkommunaler Gleichbehandlung durch den Methodenwechsel bei der Korrektur der Melderegister in Gemeinden über und unter 10.000 Einwohnern (Göß1 2018, S. 686). Die Klage wurde schließlich abgewiesen (BVerfG 2018, sowie die Kommentare in Leischner und Weigelt 2018, und Göß1 2018). Vielmehr wurde die aktuelle statistische Methodik unter der gegebenen Informationslage als geeignet und wissenschaftlich fundiert bezeichnet. Göß1 (2018) weist insbesondere darauf hin, dass ein nachgeschobener Angriff auf die Methodik nach Durchführung des Zensus künftig keine Erfolgsaussichten vor Gericht hat. Gleichzeitig führte das Bundesverfassungsgericht aus, dass die in einem Zensus gewonnenen Informationen bei der Weiterentwicklung und Optimierung der Methoden geeignet berücksichtigt werden sollten. Als Folge des Urteils (siehe BVerfG, 2015) und den weiterführenden Empfehlungen ist es daher unausweichlich, die Methodik für den Zensus 2021 mit Hilfe der aus den Zensus 2011 gewonnenen Informationen geeignet weiterzuentwickeln und anzupassen. Dies betrifft insbesondere das Stichprobenverfahren in Verbindung mit dem Methodenwechsel an der 10.000 Einwohnergrenze. Daher befassen sich die vorliegenden Untersuchungen mit der Frage, ob und inwieweit auf einen Methodenwechsel insgesamt beziehungsweise auf eine vordefinierte Einwohnergrenze verzichtet werden kann, um eine Gleichbehandlung möglichst aller Gemeinden zu erreichen. Im zweiten Kapitel erfolgt zunächst eine kurze Übersicht über die für diese Frage relevanten statistischen Methoden. Das dritte Kapitel befasst sich anschließend mit Möglichkeiten einer flexiblen und statistisch sinnvollen Festlegung der Präzisionsvorgaben, die einen Verzicht eines Methodenwechsels ermöglichen. Im vierten Kapitel wird schließlich aufgezeigt, wie unter den neuen Bedingungen 
ein Mindeststichprobenumfang für den Zensus 2021 aus statistischer Sicht abgeleitet werden kann. Es folgen eine Zusammenfassung nebst Ausblick sowie ein Anhang mit weiteren begleitenden Ergebnissen.

\section{Stichprobendesign und Schätzung im Zensus}

In diesem Kapitel werden die Stichproben- und Schätzmethodik des Zensus 2011 kurz aufgeführt. Zudem wird erläutert, welche Methoden unverändert für den Zensus 2021 übernommen werden und welche im Hinblick auf die in Kap. 1 erwähnten Probleme einer Anpassung unterzogen werden müssen. Diese Anpassungen beziehen sich insbesondere auf eine veränderte Bildung der regionalen Ausweiseinheiten sowie einer damit einhergehenden Neuformulierung der Präzisionsvorgaben. Die nachfolgenden Ausführungen orientieren sich hauptsächlich an Münnich et al. 2012a und den darin zitierten Originalquellen.

\subsection{Vorbemerkungen zur Methodik im Zensus 2011}

Ausgangspunkt für die Untersuchungen im Zensus-Stichprobenforschungsprojekt war $§ 7$ (1) des Zensusgesetzes 2011, in dem die Präzisionsziele mit einer angestrebten Genauigkeit eines einfachen relativen Standardfehlers von 0,5\% für Gemeinden mit mindestens 10.000 Einwohnern festgelegt wurden. Diese Formulierung wurde in Großstädten simultan für einzelne Stadtteile mit jeweils etwa 200.000 Einwohnern gefordert. Für das Stichprobenkonzept stellten sich daraus folgende Fragen:

- Welcher Gesamtstichprobenumfang wird benötigt, um die Präzisionsanforderungen in Form eines maximalen relativen Standardfehlers zu erfüllen?

- Wie soll der Gesamtstichprobenumfang auf die einzelnen Gemeinden beziehungsweise Stadtteile verteilt werden?

- In welchem Umfang soll in den Gemeinden unterhalb von 10.000 Einwohnern erhoben werden, um die benötigten Informationen aus dem Zensus gewinnen zu können?

- Welche Schätzmethodik soll verwendet werden, um die Präzisionsziele einzuhalten?

Um diesen Fragen nachzugehen, wurden eine Reihe von Untersuchungen auf Basis von Simulationen durchgeführt. Diese Untersuchungen hatten zunächst das Ziel, unter verschiedenen Karteileichen- und Fehlbestandsmodellen ein geeignetes Stichprobendesign zu ermitteln. Die Wahl fiel schließlich auf ein geschichtetes Design mit Anschriften als Ziehungseinheiten (siehe $\$ 7$ (3) Zensusgesetz 2011). Dabei wurde regional sowie nach Anschriftengrößenklassen geschichtet. Für die regionale Schichtung wurden die sogenannte sampling points (SMPs) als regionale Ausweiseinheit eingeführt (siehe Münnich et al. 2011, S. $22 \mathrm{ff}$.), die je nach Größe aus einer Gemeinde oder mehreren Gemeinden bestehen. Als SMP wird also im Folgenden eine regionale Schicht (beziehungsweise regionale Ausweiseinheit), die aus einer oder einem Zusammenschluss von Gemeinden gebildet wird und im Stichprobendesign als fixierte Schicht berücksichtigt wird, bezeichnet. Im Zensus 2011 
konnten wegen der Festlegung einer Mindestgröße von 10.000 Einwohnern pro SMP Allokationsprobleme auf Grund von einem zu geringen Stichprobenumfang in kleinen Ausweiseinheiten vermieden werden, obwohl jeder SMP in acht (approximativ einwohnergleiche) Anschriftengrößenklassen unterteilt wurde. Das Schätzverfahren sowie die Zuordnung der Teilstichprobenumfänge auf die SMPs und Schichten wurde a priori so festgelegt, dass die Präzisionsvorgaben eingehalten werden konnten. Wie in Kap. 3 ausführlich dargestellt, wird die Bildung der SMPs sowie die Bildung und Anzahl der Anschriftengrößenklassen beim Zensus 2021 angepasst.

\subsection{Allokation und Punktschätzung im Zensus}

Die in Kap. 1 erwähnte Notwendigkeit zur Methodikkorrektur für den Zensus 2021 betrifft hauptsächlich die Bildung der SMPs und die angestrebten Präzisionsziele. Das Grundprinzip des Allokations- und Schätzverfahren soll im Zensus 2021 analog zum Zensus 2011 durchgeführt werden. Beide Verfahren werden im Folgenden kurz erläutert. Für das geschichtete Stichprobendesign werden die SMPs im Folgenden mit $g(g=1, \ldots, G)$ und die Größenklassen mit $h=1, \ldots, H_{g}$ bezeichnet.

\subsubsection{Der kombinierte Regressionsschätzer}

Als Basisinformationen für die Schätzung im Zensus 2011 und 2021 dienen das Anschriften- und Gebäuderegister (Bezeichnung für den Zensus 2021: Steuerungsregister), in denen die Anschriften als Stichprobeneinheiten ausgewiesen sind, sowie Melderegister der Meldebehörden [siehe $§ 7$ (3) Zensusgesetz (2011), §12 (1) Entwurf zum Zensusgesetz (2021) beziehungsweise §3 Zensusvorbereitungsgesetz (2021)]. Da die Melderegister von Grund auf sehr valide Informationen liefern, dienen die registrierten Personen in den Anschriften als hochkorreliertes Hilfsmerkmal für die Schätzung der tatsächlichen Einwohnerzahl in den interessierenden SMPs. Im Zensus 2011 eignete sich bei mindestens 10.000 Einwohnern je auszuwertendem SMP ein Regressionsschätzer. Eine weitere Disaggregation der benötigten Regression in Bezug auf die Anschriftengrößenklassen ist nicht sinnvoll, da in den meisten Schichten nur ein bis zwei verschiedene Anschriftengrößen vorhanden sind.

Zur vereinfachten Darstellung der Schätzer wird nachfolgend zunächst auf den Index $g$ des $g$-ten SMP verzichtet. Mit $Y$ wird das Untersuchungsmerkmal, also die Anzahl der Einwohner, bezeichnet; dann ist $y_{i}$ die Anzahl der Einwohner in der $i$-ten Anschrift. $X$ bezeichnet das Hilfsmerkmal, $x_{i}$ stellt also die registrierten Personen in der $i$-ten Anschrift dar. Mit $\gamma_{h}=N_{h} / N$ wird der Anteil der Anschriften und mit $f_{h}=n_{h} / N_{h}$ der Auswahlsatz in der Schicht $h$ bezeichnet. Wie zuvor erläutert, dienen die Anschriftengrößenklassen als Schichtungsmerkmal. Dann ist durch

$$
\widehat{\tau}_{\mathrm{kR}}=N \cdot \sum_{h=1}^{H} \gamma_{h} \cdot\left(\bar{y}_{h}+\left(\bar{X}_{h}-\bar{x}_{h}\right) \cdot \beta\right)
$$

der Regressionsschätzwert für den SMP-Totalwert gegeben. $\beta$ bezeichnet hierbei den Regressionskoeffizienten im linearen Regressionsmodell. Formal handelt es sich um einen kombinierten Regressionsschätzer, da die Regression gemeinsam über alle 
Schichten hinweg berechnet wird. Allerdings wird die Schätzung anschließend separat für jeden SMP berechnet. Der Regressionsschätzer (1) kann auch vektoriell, also unter Verwendung mehrerer Hilfsvariablen, verwendet werden (siehe beispielsweise Cochran 1977, Särndal et al. 1992, S. 225ff. oder Lohr 2010, S. 372ff.).

Die Varianz des kombinierten Regressionsschätzers ergibt sich durch

$$
\mathrm{V}\left(\widehat{\tau}_{\mathrm{kR}}\right)=N^{2} \cdot \sum_{h=1}^{H} \frac{\gamma_{h}^{2}\left(1-f_{h}\right)}{n_{h}} \cdot\left(S_{y h}^{2}-2 \beta S_{x y h}+\beta^{2} S_{x h}^{2}\right) .
$$

Im Gegensatz zur klassischen Theorie der linearen Regression sollten im SurveyKontext Stichprobengewichte berücksichtigt werden. Daher ist die Verwendung eines varianzminimalen $\beta$ bezüglich der Varianz (2) im Vergleich zu einem durch ein ungewichtetes lineares Regressionsmodell berechnetes $\beta$, das eine uneingeschränkte Zufallsstichprobe unterstellt, vorzuziehen. Das varianzminimale $\beta$ ergibt sich unmittelbar durch die Minimierung von (2) nach $\beta$ durch

$$
\beta_{\mathrm{kR}, \min }=\frac{\sum_{h=1}^{H} \gamma_{h}^{2} \frac{1-f_{h}}{n_{h}} S_{y x h}}{\sum_{h=1}^{H} \gamma_{h}^{2} \frac{1-f_{h}}{n_{h}} S_{x h}^{2}} .
$$

Im Rahmen der einfachen Zufallsstichprobe lässt sich unter Verwendung der theoretischen Werte für die Varianzen und Kovarianzen in der Grundgesamtheit der Effizienzgewinn durch die Beziehung

$$
N^{2} \cdot \frac{1-f}{n} \cdot\left(S_{y}^{2}-2 \beta S_{x y}+\beta^{2} S_{x}^{2}\right)=N^{2} \cdot \frac{1-f}{n} \cdot S_{y}^{2} \cdot\left(1-\varrho^{2}\right)
$$

darstellen, wobei der Faktor $\varrho^{2}$ das Bestimmtheitsmaß der linearen Einfachregression ist. Bei nur einer Hilfsvariablen enspricht $\varrho$ dem Korrelationskoeffizienten zwischen Untersuchungs- und Hilfsmerkmal. Der Effizienzgewinn von freier zu gebundener Hochrechnung kann unter Verwendung der theoretischen Varianzen und Kovarianzen der Grundgesamtheit als die Höhe des Bestimmtheitsmaßes im zugehörigen Regressionsmodell angesehen werden (siehe auch Lohr 2010, S. 74ff.).

Auf die geschichtete Hochrechnung lässt sich diese einfache Darstellung der Varianzreduktion nicht direkt übertragen. Allerdings lässt sich unter Verwendung der theoretischen Varianzen und Kovarianzen die Varianz des Totalwertschätzers für die Einwohnerzahl

$$
\mathrm{V}\left(\widehat{\tau}_{\mathrm{kR}}\right)=N^{2} \cdot \sum_{h=1}^{H} \frac{\gamma_{h}^{2}\left(1-f_{h}\right)}{n_{h}} \cdot S_{y h}^{2} \cdot\left(1-\vartheta^{2}\right)
$$


mit

$$
\left(1-\vartheta^{2}\right)=1-\frac{\left(\sum_{h=1}^{H} \gamma_{h}^{2} \frac{1-f_{h}}{n_{h}} S_{y x h}\right)^{2}}{\left(\sum_{h=1}^{H} \gamma_{h}^{2} \frac{1-f_{h}}{n_{h}} S_{y h}^{2}\right) \cdot\left(\sum_{h=1}^{H} \gamma_{h}^{2} \frac{1-f_{h}}{n_{h}} S_{x h}^{2}\right)}
$$

herlieten. Die Formel (6) entspricht Formel (7.1.40) in Krug et al. (2001) und repräsentiert die Varianzreduktion zwischen der freien und gebundenen Hochrechnung. Ein Beweis für die Varianzreduktion findet sich in Anhang 1. In der Praxis wird die Varianzreduktion bei geschichteter kombinierter Regressionsschätzung aufgrund des bereits eingetretenen Schichtungseffekts im Allgemeinen kleiner ausfallen als bei der uneingeschränkten Zufallsstichprobe. Daher kann $\vartheta$ auch nicht als Korrelationskoeffizient im eigentlichen Sinne behandelt werden, sondern nur im Sinne eines allgemeineren Zusammenhangsmaßes für geschichtete Zufallsstichproben, mit dessen Hilfe sich die Varianzreduktion zwischen freier und gebundener Hochrechnung in der geschichteten Zufallsstichprobe ermitteln lässt. Wie in (4) gehen auch hier die Varianzen und Kovarianzen der Grundgesamtheit, die bei einem alleinigen Vorliegen einer Stichprobe geschätzt werden müssen, ein. Diese Größen können aber direkt aus

$$
\widehat{\operatorname{Corr}}(y, x)=\frac{\sum_{i}\left(x_{i}-\bar{x}\right)\left(y_{i}-\bar{y}\right) / \pi_{i}}{\left(\left(\sum_{i}\left(x_{i}-\bar{x}\right)^{2} / \pi_{i}\right) \cdot\left(\sum_{i}\left(y_{i}-\bar{y}\right)^{2} / \pi_{i}\right)\right)^{1 / 2}}
$$

mit $\bar{x}=\sum_{i} \frac{x_{i}}{\pi_{i}} / \widehat{N}$ ( $y$ analog) geschätzt werden; in gleicher Weise ergeben sich die korrespondierenden Varianzen (siehe Särndal et al. 1992, S. 489). Die Inklusionswahrscheinlichkeiten für die geschichtete Stichprobe sind $\pi_{i}=n_{h} / N_{h}$, wobei sich jeweils der Index $i$ in der $h$-ten Schicht befindet. Die Varianzen, Kovarianzen und der Korrelationskoeffizient lassen sich somit auf allgemeine Stichprobenmodelle übertragen. Die Varianzreduktion (5) lässt sich für allgemeine Stichprobenmodelle in analoger Vorgehensweise zu Anhang A herleiten.

\subsubsection{Die Box-Constraint optimale Allokation}

In Anlehnung an die Methodik des Zensus 2011 wird auch für den Zensus 2021 eine optimale Allokationsmethode mit Box-Constraints (kurz: BCopt) vorgeschlagen. Da sich die Vorgehensweise im Zensus 2011 (siehe Münnich et al. 2012a, Abschn. 2.2.4) bewährt hat, soll sie in ähnlicher Form auch für den Zensus 2021 angewendet werden. Die Allokationsmethode und deren algorithmische Umsetzung wird in Gabler et al. (2012) und Münnich et al. 2012b ausführlich erläutert. Außerdem sind Erweiterungen wie eine ganzzahlige optimale Allokation mit Box-Constraints in Friedrich et al. (2015, Anschriften können nur komplett gezogen werden) sowie eine multivariate optimale Allokation mit Box-Constraints in Friedrich et al. (2018, mehrere interessierende Variable in der Allokation) und Rupp (2018, Kap. 4) zu finden. Grundsätzlich sind folgende Anforderungen zwingend zu erfüllen: 
- Einhaltung von zuvor definierten SMP-spezifischen Präzisionsanforderungen für die Einwohnerzahl in den SMPs;

- Robustheit der Allokation gegenüber den interessierenden Fragestellungen und Ziele;

- obere und untere Schranken für die Auswahlsätze pro Anschriftengrößenklasse;

- Einhaltung eines gegebenen Gesamtstichprobenumfangs.

Da alle SMPs in der Optimierung berücksichtigt werden sollen, wird eine gewichtete Summe der quadrierten RRMSEs der SMP-spezifischen Schätzer minimiert. Diese erlaubt eine gleichmäßige Optimierung über alle SMPs hinweg. Für den Horvitz-Thompson Schätzer $\widehat{\tau}_{\mathrm{HT}}$ und die SMP-spezifischen Horvitz-Thompson Schätzer $\widehat{\tau}_{\mathrm{HT}, \mathrm{g}}$ ist diese Funktion gegeben durch

$$
\sum_{g=1}^{G} w_{g} \operatorname{RRMSEs}\left(\widehat{\tau}_{\mathrm{HT}, \mathrm{g}}\right)^{2}=\sum_{g=1}^{G} \frac{w_{g}}{\tau_{g}^{2}} \sum_{h=1}^{H_{g}} \frac{N_{g h}^{2} S_{g h}^{2}}{n_{g h}}\left(1-\frac{n_{g h}}{N_{g h}}\right) .
$$

Die Anzahl der Anschriften pro SMP und Schicht wird mit $N_{g h}$ beziehungsweise $n_{g h}$ für die Gesamtheit beziehungsweise die Stichprobe bezeichnet. $S_{g h}$ ist die jeweilige schicht- und SMP-spezifische Varianz, berechnet mit Hilfe der zu Verfügung stehenden Registerwerte. $\tau_{g}$ ist die Anzahl der registrierten Personen in SMP $g$ (mit Erstwohnsitz). $w_{g}$ bezeichnet einen SMP-spezifischen Gewichtungsvektor, mit dessen Hilfe eine Präzisionsvorgabefunktion in die Methodik integriert werden kann. Zur genaueren Analyse der Wahl des Gewichtungsvektors verweisen wir auf Kap. 3. Da jedoch, wie in Abschn. 2.2.1 erläutert, der kombinierte Regressionsschätzer $\widehat{\tau}_{\mathrm{kR}}$ gemäß (1) bestimmt werden soll, muss die Funktion in (8) an die Varianz des kombinierten Regressionsschätzers (2) angepasst werden. In dem Zusammenhang spielt insbesondere der SMP-spezifische Varianzreduktionsfaktor $\left(1-\vartheta_{g}^{2}\right)$ nach Formel (6) eine Rolle. Unter Annahme bekannter Varianzreduktionsfaktoren ergibt sich somit die zu minimierende Funktion

$$
\sum_{g=1}^{G} w_{g} \operatorname{RRMSEs}\left(\widehat{\tau}_{\mathrm{HT}, \mathrm{g}}\right)^{2} \cdot\left(1-\vartheta_{g}^{2}\right)=\sum_{g=1}^{G} \frac{w_{g}}{\tau_{g}^{2}} \sum_{h=1}^{H_{g}} \frac{N_{g h}^{2} S_{g h}^{2}}{n_{g h}}\left(1-\frac{n_{g h}}{N_{g h}}\right) \cdot\left(1-\vartheta_{g}^{2}\right)
$$

in Analogie zu (8). Für den Zensus 2021 muss die Verwendung der Varianzreduktionsfaktoren $\left(1-\vartheta_{g}^{2}\right)$ innerhalb der BCopt Allokation jedoch kritisch diskutiert werden, da diese auf einer Schätzung mittels einer einzigen Stichprobe (der Zensusstichprobe aus dem Jahr 2011) basieren. Dies führt zu nicht quantifizierbaren Ungenauigkeiten; Abhilfe würden etwaige Hilfsinformationen aus dem Zensus 2011 bringen, die aber nicht mehr zu Verfügung stehen. Zudem würde die Verwendung der Varianzreduktionsfaktoren innerhalb des Allokationsprozesses eine Ungleichbehandlung der SMPs implizieren, was insbesondere auch eine Ungleichbehandlung der Gemeinden nach sich ziehe. Alternativ kann für die Allokation ein konstanter mittlerer Varianzreduktionsfaktor für alle SMPs verwendet werden, sodass dieser zwar die Größe des Gesamtstichprobenumfangs bedingt, aber keinen direkten Einfluss auf die Allokation hat. Dieses Konzept wurde auch schon im Jahr 2011 durchgeführt. Zur Ermittlung des benötigten Gesamtstichprobenumfangs werden die 
Varianzreduktionsfaktoren weiterhin verwendet, da sie dafür von entscheidender Bedeutung sind (siehe dazu Kap. 4). Für die Minimierung von (9) kann der konstante Term vernachlässigt werden. Somit ergibt sich folgende Zielfunktion:

$$
\min _{n_{g h}} \sum_{g=1}^{G} \sum_{h=1}^{H_{g}} \frac{w_{g}}{\tau_{g}^{2}} \frac{N_{g h}^{2} S_{g h}^{2}}{n_{g h}}\left(1-\vartheta_{g}^{2}\right) .
$$

Unter Berücksichtigung der Restriktionen für den Gesamtstichprobenumfang (Gesamtauswahlsatz von $\theta \in[0,1])$ und der Einschränkung der unteren und oberen Auswahlsätze pro SMP und Schicht ergibt sich schließlich folgendes restringiertes Optimierungsproblem:

$$
\begin{aligned}
& \min _{n_{g h}} \sum_{g=1}^{G} \sum_{h=1}^{H_{g}} \frac{w_{g}}{\tau_{g}^{2}} \frac{N_{g h}^{2} S_{g h}^{2}}{n_{g h}}\left(1-\vartheta_{g}^{2}\right) \\
& \text { s.t. } \sum_{g=1}^{G} \sum_{h=1}^{H_{g}} n_{g h} \mathrm{PpA}_{g h}=\tau \cdot \theta \\
& \quad m_{g h} \leq n_{g h} \leq M_{g h} \forall g=1, \ldots, G \text { und } h=1, \ldots, H_{g},
\end{aligned}
$$

wobei $\mathrm{PpA}_{g h}$ die durchschnittliche Anzahl von Personen in einer Anschrift für SMP $g$ und Schicht $h$ ist. $\tau$ ist die Anzahl der im Erstwohnsitz registrierten Personen in Deutschland. $m_{g h}$ und $M_{g h}$ bezeichnen die unteren und oberen Schranken für die Anzahl der zu ziehenden Anschriften für SMP $g$ und Schicht $h$. Durch eine einfache Substitution mit $N_{g h}$ lassen sich daraus SMP- und schichtspezifische Grenzen für untere und obere Auswahlsätze definieren.

\subsection{Abschließende Bemerkungen zum Zensus 2011}

\subsubsection{Festlegungen für den Zensus 2011}

Im Zensus 2011 wurde a priori der Gesamtstichprobenumfang vorgegeben. Es sollten gemäß Stichprobenverordnung des Zensusgesetzes 9,6\% der Bevölkerung Deutschlands befragt werden, was $\theta$ in Gleichung (11) entspricht. Zudem wurde für Gemeinden größer als 10.000 Einwohnern (bildeten jeweils ein SMP) ein maximaler relativer Standardfehler von 0,5\% festgelegt. Dazu wurden vier Gemeindegrößenklassen definiert, für die jeweils eigene obere und untere Schranken für die Auswahlsätze definiert wurden. In Gemeinden unterhalb von 10.000 Einwohnern (mehrere Gemeinden bildeten ein SMP) wurde ein konstanter Auswahlsatz in Höhe von $5 \%$ gewählt. Dieser diente zur Schätzung von Variablen, die nicht unmittelbar die Einwohnerzahl betreffen. Oberhalb von 10.000 Einwohnern wurden zwei weitere Grenzen festgelegt. Bis unter 30.000 Einwohner wurde ein Auswahlsatz zwischen 5\% und 50\%, von 30.000 bis unter 100.000 Einwohnern $4 \%$ bis $40 \%$ und schließlich für größere Gemeinden $2 \%$ bis $40 \%$ erlaubt. Grundlage hierfür war unter anderem das Gebot der Sparsamkeit. Gleichzeitig erlaubt diese Festlegung aufgrund einer beschränkten Variation der Survey-Gewichte auf den Faktor 25 auch noch die Anwendung von modellbasierten Verfahren. Die konkrete Anzahl der darauf resultierenden zu ziehenden Anschriften wurde in $\$ 3$ (2) der Stichprobenverordnung nach Bundesländern aufgegliedert manifestiert. 


\subsubsection{Was haben wir aus dem Zensus 2011 gelernt}

Die Vorgabe eines auf Basis des Zensustests festgelegten relativen Standardfehlers in Kombination mit einem maximalen Stichprobenumfang beinhaltet eine konfliktäre Vorgabe, da sowohl der maximale Stichprobenumfang wie auch die Mindestqualität a priori vorgegeben wurden. Durch die abgeschwächte Formulierung [...] mit einer angestrebten Genauigkeit [...] [\$7 (1) Absatz 1 Zensusgesetz (2011)] wurde diesem Konflikt in der Gesetzgebung Rechnung getragen. Trotzdem führten die beiden konfliktären Vorgaben in einigen SMPs zu einer Abweichung von der angestrebten Präzision. Für den Zensus 2021 sollte insbesondere der Umstand des ausschließlichen Vorliegens von Stichprobendaten mit einer daraus implizierten Unsicherheit noch stärker berücksichtigt werden. Als mögliche Abhilfe werden in Kap. 4 zwei Methoden zur Berechnung eines Sicherheitsaufschlagfaktors für den Gesamtstichprobenumfang genauer eruiert.

Die Nachuntersuchungen im Zensusvalidierungsprojekt zeigten weitere kritische Effekte (siehe Münnich et al. 2013a). Einerseits ergaben sich mehr Schichtwechsler als erwartet. Als Schichtwechsler werden in diesem Kontext Stichprobeneinheiten bezeichnet, die zum Zeitpunkt der Erstellung der Auswahlgrundlage einer anderen Schicht (Anschriftengrößenklasse) zugeordnet wurden, als der Schicht, der sie auf Basis der Stichprobeninformationen angehören sollten. Der Grund dafür ist der Umstand, dass die Schichteinteilung relativ früh erfolgte und somit größere Abweichungen als erwartet auftraten. Ebenso zeigten sich stärkere Ausreißer in der obersten Schicht, also bei den großen Anschriften. Das Hauptziel des Validierungsprojektes war es jedoch, den Einfluss der unerwartet hohen Anzahl an Nullanschriften auf die Methodik zu untersuchen. Als Ergebnis erwies sich die Methodik als robust, sodass die Empfehlungen zur Methodik nicht angepasst werden mussten. Allerdings war ein Effizienzverlust der Schätzungen erwartbar. Dieser Verlust lässt sich für den Zensus 2021 reduzieren. Maßnahmen zur Reduktion sind beispielsweise die Minimierung des Zeitraums zwischen der Erstellung der Auswahlgrundlage durch das Anschriften- und Gebäuderegister (Steuerungsregister) und der Stichprobenziehung sowie die Prüfung der im Anschriften- und Gebäuderegister geführten Anschriften auf Wohnraum. Darüber hinaus zeigte sich, dass die größenabhängige Ungleichbehandlung der SMPs bezüglich des Mindestauswahlsatzes zu vereinzelten Qualitätsdifferenzen führen konnte, was eine Angleichung des Mindestauswahlsatzes nahelegte.

Weitere Analysen für den Zensus 2011 werden in Münnich et al. (2015) zusammengefasst. Hier wird untersucht, welche Methoden der Qualitätsmessung herangezogen werden können, wenn bei Schätzungen von Tabellen für sogenannte Ziel2-Variablen fehlende Werte auftreten. Als Ziel-2-Variablen werden im Kontext des Zensus Zusatzmerkmale, die nicht aus den Melderegistern abzuleiten sind, bezeichnet. Der Großteil dieser Merkmale betrifft bildungs- und erwerbsrelevante Informationen, wie zum Beispiel höchster Schulabschluss oder Selbstständigkeit (vgl. Berg und Bihler 2011, Kap. 2). Aktuell sind die Berechnungen von Varianzschätzungen unter Imputation so aufwändig, dass diese nur in eingeschränktem Umfang durchgeführt werden können. Varianzschätzungsfunktionale können hier möglicherweise in sehr effizienter Weise gute Proxies liefern. 
In Flensburg wurde bei den Ein- bis Siebenjährigen ein unerwartet hoher Anteil an Jungen festgestellt (siehe Kap. 1). Als Grund dafür ist ausschließlich die Optimierung der Methodik auf die Schätzung der Gesamteinwohnerzahl innerhalb der SMPs zu nennen. Die Verwendung des GREG-Schätzers für kleine Untergruppen ist dabei wenig empfehlenswert, da im Allgemeinen bei Gewichtungsverfahren kleine Teilgruppen auffällige Ergebnisse aufweisen können. Mit Hilfe eines verallgemeinerten Kalibrierungsschätzer (siehe Burgard et al. 2019) können auch solche Teilgruppen sowie sehr kleine Nachweiseinheiten, sog. Small Areas (siehe auch Rao und Molina 2015, Münnich et al. 2013b und Tzavidis et al. 2018), besser berücksichtigt werden. Das Verfahren beinhaltet ein implizites Editing, da bei der Bestimmung des optimalen Gewichtevektors automatisch auch auffällige Abweichungen von den Registerwerten aufgezeigt werden. Methodische Implikationen als Folge der Nachuntersuchungen zu Ziel-2 sowie als Folge des Ausweises auf Teilgruppen sehr kleiner Nachweiseinheiten werden im Rahmen dieses Artikels nicht weiter untersucht.

\subsubsection{Untersuchungsziel des Artikels}

In Folge des Urteils vom Bundesverfassungsgerichts (siehe BVerfG 2018) und den zuvor ausgeführten Aspekten werden nachfolgend drei Fragenkomplexe im Hinblick auf die Methodik für den Zensus 2021 untersucht.

1. In den Kap. 3.1, 3.2 und 3.3 wird die Frage diskutiert, ob und wie man die aktuellen Präzisionsanforderungen auch auf Gemeinden mit weniger als 10.000 Einwohnern erweitern kann. Hierzu muss zunächst geklärt werden, ob und inwieweit der relative Standardfehler auf die sehr heterogenen Größenstrukturen der Gemeinden in Deutschland angewendet werden kann. Das führt zu einer Diskussion zwischen absoluten und relativen Standardfehler beziehungsweise zu einer Flexibilisierung der bisher verwendeten Präzisionsanforderungen für Gemeinden unterhalb 10.000 Einwohnern. Dies gilt insbesondere in Hinblick darauf, dass sich bei Gemeinden mit nur sehr wenigen Anschriften klassische Stichprobenkonzepte nicht adäquat anwenden lassen.

2. Anschließend wird in Kap. 3.4 die Bedeutung der Varianzreduktionsfaktoren geschätzt aus der Zensusstichprobe aus 2011 - für die Präzision der Schätzungen der Bevölkerungszahlen in den Gemeinden diskutiert. Ebenso wird geklärt, inwieweit der kombinierte Regressionsschätzer sinnvoll auch auf kleine Gemeinden angewendet werden kann. Daraus ergeben sich weitere Nebenbedingungen, die in Empfehlungen für die methodische Vorgehensweise im Zensus 2021 münden.

3. Die aus der sehr heterogenen Gemeindestruktur resultierende Frage, ob Vollerhebungsschichten verwendet werden sollen oder ob geeignete Zusammenfassungen von Gemeinden verwendet werden können, wird in den Kap. 3.5 und 3.6 untersucht. Letzteres führt zu einer Neukonstruktion der für den Zensus 2011 formulierten SMPs mit dem Ziel eines effizienten und widerspruchsfreien Stichprobenziehungskonzeptes. Abschließend werden die verschiedenen Ansätze miteinander vergleichend diskutiert.

Ausgehend von den Untersuchungen zu den zuvor beschriebenen drei Fragenkomplexen wird in Kap. 4 schließlich der benötigte Stichprobenumfang diskutiert. Auf 
Basis der Erfahrungen aus dem Zensus 2011, bei dem eine strikte Verwendung der Stichprobeninformationen zu Karteileichen und Fehlbeständen aus dem Zensustest zu einer teilweisen Nichterfüllung der Präzisionsziele geführt hat und die Festlegung eines Stichprobenumfangs von 7,9 Mio. Einwohnern soeben ausgereicht hat, wird diskutiert, welche statistischen Modellierungen zu einer konservativen Schätzung des benötigten Mindeststichprobenumfangs führen können. Abschließend werden die Ergebnisse in Kap. 5 in Form von Empfehlungen für den Zensus 2021 zusammengefasst.

\section{Methodische Betrachtung der Zensus-Zielvorgaben}

\subsection{Vorüberlegungen zur Anpassung der Präzisionszielvorgaben}

In Folge einer Vermeidung der 10.000er-Grenze im Zensus 2021 stellt sich zunächst die Frage, wie die Qualität der Schätzung der Bevölkerungszahl angesichts der sehr heterogenen Größen der Gemeinden in Bezug auf Personen und Anschriften sinnvoll festgesetzt werden kann (siehe Kap. 3.6 zur Analyse der Gemeindestrukturen). Zur Vereinfachung wird dabei immer von Gemeinden als regionale Ausweiseinheit gesprochen. Generell ist die Theorie gültig für beliebig gebildete SMPs.

Im Allgemeinen werden im Europäischen Statistischen System unterschiedliche teilweise flexible Formulierungen für relative Standardfehler verwendet, die sowohl von den Anteilswerten der interessierenden Merkmale als auch von der Größe der Basispopulation abhängen (siehe beispielsweise Eurostat 2013, S. 18 ff.). Dabei wird im Speziellen eine relative Vorgehensweise wie folgt dargestellt (siehe Eurostat 2013, S. 7):

The estimated standard error [...] shall not exceed 2 percentage points of the overall proportions and shall not exceed 5 percentage points for the proportions related to the different subgroups of the population, where these subgroups constitute at least $10 \%$ of the total population in the scope of the survey.

Insbesondere für europäische Haushaltsstichprobenerhebungen wurde in den Draft Regulations des European Statistical System Committee (siehe European Statistical System Committee 2015, S. 28f.) folgende Beziehung zur Berechnung des Stichprobenumfangs vorgeschlagen:

$$
n_{g ; S R S}=f\left(N_{g}\right)=\alpha \cdot \sqrt{N_{g}}+\beta .
$$

Dieser Vorschlag entspricht der Formulierung in Verma (1991). Der Parameter $\alpha$ adjustiert den SMP-spezifischen Stichprobenumfang für die jeweilige Erhebung und orientiert sich an der Wichtigkeit der Variablen im Sinne einer Mindestpräzision des Totalwertschätzers für diese Variable. $\beta$ gibt einen Mindeststichprobenumfang an. $n_{g ; S R S}$ repräsentiert dann den zu wählenden Stichprobenumfang bei vorliegender uneingeschränkter Zufallsstichprobe für Gemeinde/SMP $g$. Dieser muss anhand des konkreten Stichprobendesigns unter Zuhilfenahme des dazugehörigen Varianzschätzfunktionals beziehungsweise Design-Effekts umgerechnet werden. In Euro- 
pean Statistical System Committee (2015, S. 28 f.) wird dies speziell für ausgewählte Haushaltsstichproben in der EU mit Hilfe konkreter Parameter aufgeführt. Ähnliche Konzepte, allerdings in anderem Kontext vergleichender Stichproben, wurden bereits in Kish (1987) aufgestellt.

Das beschriebene Konzept der zu erzielenden Schätzqualität gilt es nachfolgend geeignet für den Zensus 2021 zu formulieren, wobei die Präzisionsanforderungen eines relativen Standardfehlers von $0,5 \%$ für Gemeinden mit mehr als 10.000 Einwohnern aus dem Zensus 2011 beibehalten werden sollen. Darüber hinaus ist zu untersuchen, in welcher Form dieses Verfahren auf die Gemeinden mit weniger als 10.000 Einwohner adäquat zu übertragen ist. Aufgrund des riesigen Gesamtspektrums der Gemeindegrößen ist eine alleinige Festlegung einer Fehlerobergrenze durch den relativen Standardfehler ausgeschlossen. Stattdessen bedarf es einer kombinierten Betrachtung des relativen und absoluten Standardfehler. Eine absolute Formulierung ist gerade bei extrem kleinen Gemeinden, in denen aufgrund einer zu geringen Anzahl an zu ziehenden Anschriften klassische Stichprobenziehungen nicht mehr anwendbar sind, angebracht.

Um diese Konzepte für kleine und größere Gemeinden innerhalb einer Präzisionszielfunktion zu vereinen, wird die Definition eines glatten Übergangs von absoluten zum relativen Standardfehler innerhalb eines Übergangsintervalls vorgeschlagen. Zur Implementation eines homogenen Konzeptes sind die Einhaltung folgender Eigenschaften für die gesamte Präzisionszielfunktion von besonderer Bedeutung:

Stetige Differenzierbarkeit, Monotonie und Linksgekrümmtheit.

Diese Eigenschaften sollen sowohl für eine Betrachtung des absoluten als auch des relativen Standardfehlers in Abhängigkeit der Gemeindegröße gelten. Als Ansätze für das Übergangsintervall $[a, b]$ zwischen den Gemeindegrößen $a$ und $b$ werden in diesem Artikel monotone Splines und Bézierkurven vorgeschlagen. Als Übergangsintervall wird der Bereich der Gemeindegröße bezeichnet, dem kein fester absoluter und kein fester relativer Standardfehler zugeordnet ist.

Generell sind Splines zusammengesetzte, stückweise definierte Polynome, die häufig stetig differenzierbar und falls gewünscht auch als monotone Funktionen definiert werden können. Für detaillierte Definitionen, Beschreibungen und Analysen verweisen wir auf Schumaker (2007, Kap. 4). Monotone kubische Splines auf dem Übergangsintervall $[a, b]$ sind definiert als Funktion $S:[a, b] \rightarrow \mathbb{R}$ mit

$$
S(t)=\sum_{j=0}^{m-1} \sum_{i=0}^{3} c_{i j}\left(t-\gamma_{j}\right)_{+}^{i},
$$

wobei das Intervall $[a, b]$ in $m+1$ disjunkte Teilintervalle $\left[\gamma_{j}, \gamma_{j+1}\right](j=0, \ldots, m-$ 1) aufgeteilt ist mit $a=\gamma_{0}$ und $b=\gamma_{m}$. Daher gilt

$$
[a, b]=\bigcup_{j=0}^{m-1}\left[\gamma_{j}, \gamma_{j+1}\right] .
$$


Des Weiteren ist $\left(t-\gamma_{j}\right)_{+}^{i}$ definiert als

$$
\left(t-\gamma_{j}\right)_{+}^{i}:=\left\{\begin{array}{cl}
0 & \text { für } t<\gamma_{j} \\
\left(t-\gamma_{j}\right)^{i} & \text { für } t \geq \gamma_{j}
\end{array} .\right.
$$

Zur Bestimmung eines kubischen Splines sind daher neben dem Start- und Endpunkt auch die sogenannten Knotenpunkte $\gamma_{1}, \ldots, \gamma_{m-1}$ sowie die Konstanten $c_{i j}$ zu bestimmen. Zur Erreichung einer stetig differenzierbaren Gesamtfunktion werden an den jeweiligen Start-, End- und Knotenpunkten stetig differenzierbare Übergänge gefordert. Die a priori Auswahl dieser Parameter erfordert somit eine Vielzahl von Festlegungen, um einen solchen Spline in der gewünschten Form zu erhalten.

Neben den Splines sind Bézierkurven ein geeignetes Instrument zur stetig differenzierbaren Verbindung zweier Kurven. Ursprünglich wurden sie zur Verwendung in grafischen Darstellungen entwickelt. Für weitere Details verweisen wir auf Weisstein (2018). Zur Definition seien dazu die Punkte $P_{0}:=(a, f(a))$ und $P_{2}:=(b, f(b))$ der Start- und Endpunkt des Übergangsintervalls $[a, b]$, wobei die Funktion $f$ der Gemeindegröße den absoluten beziehungsweise relativen Standardfehler zuordnet. Des Weiteren sei $P_{1}$ der Schnittpunkt der beiden Tangenten an die Punkte $P_{0}$ und $P_{2}$ (siehe Abb. 1). Damit ist die hier verwendete quadratische Bézierkurve definiert durch die Funktion $B:[0,1] \rightarrow \mathbb{R}^{2}$ mit

$$
B(t)=\left(P_{0}-2 P_{1}+P_{2}\right) t^{2}+2\left(P_{1}-P_{0}\right) t+P_{0}
$$

für $t \in[0,1]$. Die Punkte $B(t)$ geben dann die Punkte im Raum $\mathbb{R}^{2}$ an, aus denen sich die Bézierkurve bilden lässt. Durch das Umformen der Gleichung

$$
\left(\begin{array}{c}
N(t) \\
\operatorname{SE}(t)
\end{array}\right):=B(t)
$$

nach $\operatorname{SE}(N)$ ergibt sich in dieser Schreibweise eine geschlossene Form der Bézierfunktion für den relativen beziehungsweise absoluten $\operatorname{Standardfehler~} \operatorname{SE}(N)$ in Abhängigkeit der Gemeindegröße $N$. Im Vergleich zu den Splines sind zur Berechnung der Bézierkurve nur der Start- und Endpunkt, sowie die Steigung (fixer absoluter und relativer Standardfehler) in diesen Punkten notwendig. Dies ermöglicht eine einfache Umsetzung als Präzisionszielfunktion unabhängig von a priori zu wählenden Parametern. Aus diesem Grund wird die Verwendung von Bézierkurven empfohlen. Um die zuvor genannten Eigenschaften (für absoluten und relativen Standardfehler) zu erfüllen, werden die Bézierkurven auf Basis der Betrachtung von Gemeindegröße zum absoluten Standardfehler gebildet. Abweichend davon ist in Abb. 1 zur besseren Veranschaulichung die Betrachtung bezüglich des relativen Standardfehlers abgebildet. Das generelle Vorgehen zur Bildung des Splines und der Bézierkurve unterscheidet sich dabei nicht von dem Vorgehen bei der Betrachtung bezüglich des absoluten Standardfehlers. Auf die Wahl der Parameter wird im folgenden Unterkapitel vertieft eingegangen.

Generell stellen Bézierkurven einen mathematisch definierten Übergang vom Bereich absoluter Präzisionsvorgaben zum Bereich relativer Präzisionsvorgaben dar. 

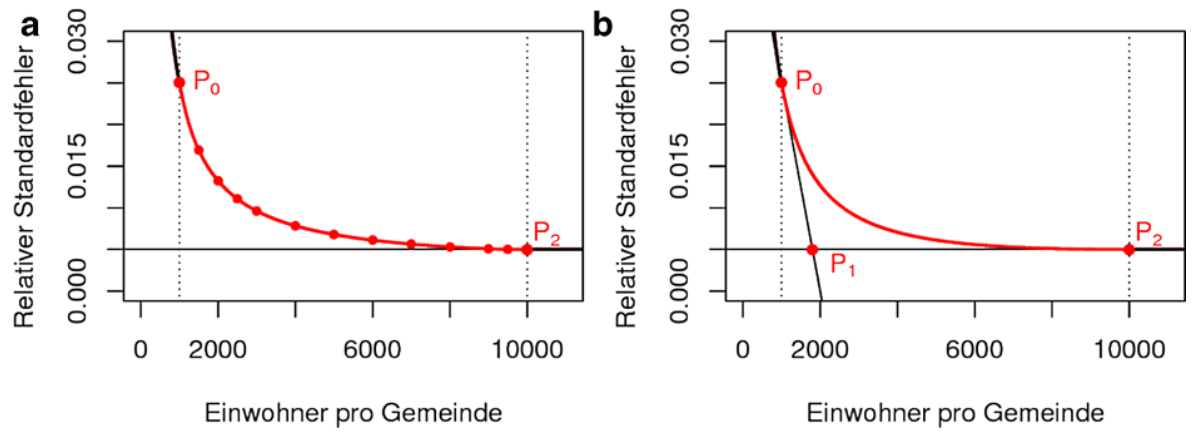

Abb. 1 Vergleich Spline und Bézierkurve in Abhängigkeit der Gemeindegröße vom relativen Standardfehler. Übergangsintervall $[a, b]=[1.000,10.000]$; absoluter Standardfehler von 25 für Gemeinden kleiner als $a=1.000$ Einwohner; relativer Standardfehler von $0,5 \%$ für Gemeinden größer als $b=10.000$ Einwohnern.

Diese sind gut geeignet, um den Ausgleich zweier konkurrierender Ziele unter vordefinierten Nebenbedingungen zu erreichen, da sie Eigenschaften wie Glattheit, strenge Monotonie und Linksgekrümmtheit erfüllen. Bei geeigneter Definition gelten diese Eigenschaften sowohl für die Betrachtung bezüglich des absoluten, wie auch bezüglich des relativen Standardfehlers.

\subsection{Bestimmung der Parameter der Präzisionszielfunktion}

Als Antwort auf die Kritik an der Methodik des Zensus 2011 bezüglich der 10.000er Grenze ist die Wahl einer variablen Präzisionszielfunktion, wie sie in Abschn. 3.1 motiviert wurde, unumgänglich. Als Instrument für das Übergangsintervall zwischen dem fixen absoluten und fixen relativen Standardfehler wurde die Verwendung von Bézierkurven vorgeschlagen, die einen glatten Übergang gewährleisten können. Nachfolgend sollen geeignete Parameter für die Definition der Bézierkurven konkretisiert werden.

Die folgenden Abb. 2 und 3 zeigen sechs Szenarien für die Definition der Bézierkurven in Abhängigkeit von dem maximal erlaubten absoluten Standardfehler (SE) für kleine Gemeinden und dem Startpunkt $a$ des Übergangsintervalls, also der Gemeindegröße, ab welcher nicht mehr nur der maximale absolute Standardfehler zu beachten ist. Die Bezeichnung lautet bez_[a]_[SE]. In Anlehnung an den Zensus 2011 wird der Endpunkt des Übergangsintervalls auf $b=10.000$ Einwohner pro Gemeinde festgesetzt, wobei ab dieser Gemeindegröße eine Präzision von einem relativen Standardfehler (rSE) von $0,5 \%$ gefordert wird. Folgende Szenarien werden unterschieden:

(a) bez_100_2.5: Startpunkt $a=100$, darunter absoluter Standardfehler von 2,5

(b) bez_100_5: Startpunkt $a=100$, darunter absoluter Standardfehler von 5

(c) bez_100_10: Startpunkt $a=100$, darunter absoluter Standardfehler von 10

(d) bez_1000_15: Startpunkt $a=1.000$, darunter absoluter Standardfehler von 15

(e) bez_1000_25: Startpunkt $a=1.000$, darunter absoluter Standardfehler von 25

(f) bez_1000_40: Startpunkt $a=1.000$, darunter absoluter Standardfehler von 40 



Abb. 2 Relativer Standardfehler bei Bézierkurven in Abhängigkeit der Gemeindegröße

In Abb. 2 ist die Abhängigkeit der Gemeindegröße (Einwohner) zum relativen Standardfehler abgebildet, Abb. 3 zeigt die Abhängigkeit zum absoluten Standardfehler.

Zur Begründung der Wahl der Parameter für das Übergangsintervall $[a, b]$, dem vorgegebenen maximalen SE für Gemeinden kleiner als $a$ Einwohner sowie dem konstanten maximalen rSE von $0,5 \%$ für Gemeinden größer als $b$ Einwohner lassen sich folgende Argumente anführen. In erster Linie ist die Wahl eine politische Entscheidung, mit der die gewünschte Genauigkeit der Umfrage festgelegt wird und welche nur im Zusammenspiel mit der zugrundeliegenden Gemeindestruktur
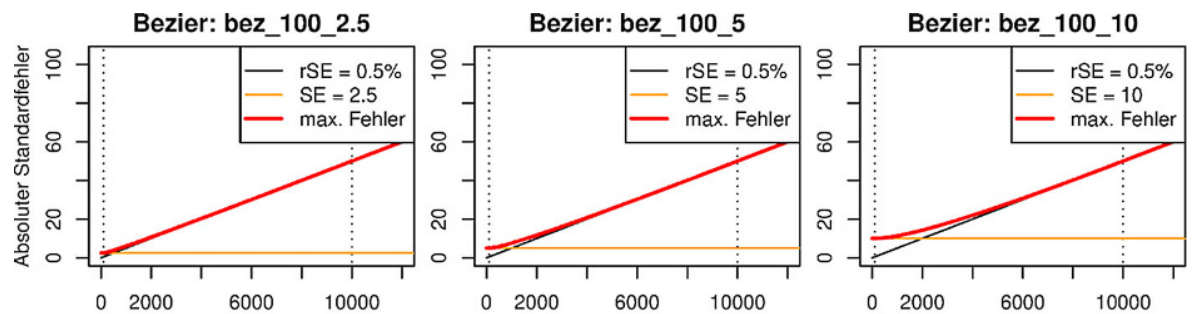

Bezier: bez_1000_15
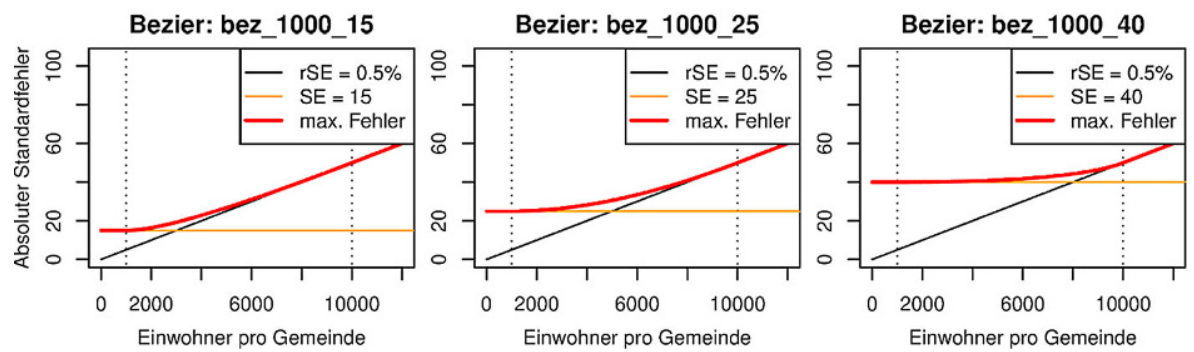

Abb. 3 Absoluter Standardfehler bei Bézierkurven in Abhängigkeit der Gemeindegröße 
sinnvoll getroffen werden kann. Grundsätzlich sollte dabei die Qualität im Zensus 2011 nicht verschlechtert werden. Da im Zensus 2021 alle Gemeinden der gleichen Methodik unterliegen, ist zu ergründen, ab welcher Gemeindegröße eine explizite Fehlerrechnung mittels des relativen Standardfehlers sinnvoll erscheint. Dabei sind unter anderem theoretische Überlegungen von Kish und Verma (vgl. Abschn. 3.1), Ganzzahligkeitseffekte bei kleinen Gemeinden und die Stabilität der GREG-Schätzungen (vgl. Abb. 11) zu beachten. Diese Aspekte und die Tatsache, dass Anschriften und nicht Einwohner die Stichprobeneinheiten bilden, lässt auf eine sinnvolle Wahl der unteren Intervallgrenze von $a=1.000$ schließen. Eine Wahl von $a=100$ würde diesen Argumenten nicht vollständig gerecht werden.

Anhand dieser Analysen und der Simulationsergebnisse, die in Verlauf dieses Kapitels sowie in Kap. 4 vorgestellt werden, wird die Wahl des für kleine Gemeinden geforderten maximalen absoluten Standardfehler von 15 und 25 als zu präferierende Größen vorgeschlagen [also Präzisionszielfunktionen (d) und (e)]. Bei einem erlaubten absoluten Standardfehler von 40 würde die Schätzqualität in kleinen Gemeinden erheblich leiden. Die folgenden Auswertungen beschränken sich deswegen auf diese beiden Präzisionsvorgaben; teilweise wird nur auf die Präzisionszielfunktion (d) bez_1000_15 eingegangen.

\subsection{Anwendung der Präzisionszielfunktion}

Mit Hilfe der in Abschn. 3.2 definierten Präzisionszielfunktion kann nun jeder Gemeinde $g$ ein maximal erlaubter relativer Standardfehler $\mathrm{rSE}_{g}$ und absoluter Standardfehler $\mathrm{SE}_{g}$ zugeordnet werden. Um einerseits diesen Standardfehler einzuhalten, andererseits aber gleichzeitig eine kompensatorische optimale Allokation (siehe Abschn. 2.2) durchzuführen, wird folgendes Verfahren angestrebt:

1. Zur Berechnung des unter Berücksichtigung der Präzisionszielfunktion benötigten Gesamtstichprobenumfangs wird das sogenannte Amin-Verfahren separat für jede einzelne Gemeinde angewendet. Dabei werden die aus der Zensusstichprobe von 2011 geschätzten Varianzreduktionsfaktoren $\left(1-\vartheta^{2}\right)$ verwendet. Der Amin Algorithmus in Gemeinde $g$ ist ein iteratives Verfahren, was als Startpunkt die auf Basis der unteren Auswahlsätze definierten minimalen schichtspezifischen Stichprobenumfänge $m_{g 1}, \ldots, m_{g} H_{g}$ verwendet. Diese werden sukzessive in der Schicht, welche die größte Varianzreduktion für den gemeindespezifischen kombinierten Regressionsschätzer $\widehat{\tau}_{\mathrm{kR}, g}$ mit sich bringt, um Eins erhöht. Begrenzt ist die Erhöhung durch die maximalen schichtspezifischen Stichprobenumfänge $M_{g 1}, \ldots, M_{g H_{g}}$. Der Algorithmus stoppt, wenn die gegebene Präzision eingehalten wird beziehungsweise wenn der maximale Stichprobenumfang in jeder Schicht erreicht ist.

2. Um eine kompensatorische optimale Allokation zu erzielen, wird der aus Amin resultierende Gesamtstichprobenumfang nun als Basis für die in Abschn. 2.2.2 beschriebene BCopt Allokation verwendet (ggf. mit einem noch zu spezifizierenden Sicherheitsaufschlagfaktor, siehe Kap. 4). Dabei werden die geschätzten Varianzreduktionsfaktoren $\left(1-\vartheta^{2}\right)$ nicht pro Gemeinde separat berücksichtigt, sondern über alle Gemeinden hinweg gemittelt. Dies soll ein Ungleichbehandlung einzel- 
Tab. 1 Quantile der Gemeindegrößen (inklusive Stadtteile) aller 11.377 Gemeinden im synthetischen Datensatz von 2011

\begin{tabular}{lccccc}
\hline Quantile & $0 \%$ & $25 \%$ & $50 \%$ & $75 \%$ & $100 \%$ \\
Gemeindegröße & 9 & 644 & 1.692 & 5.139 & 365.598 \\
\hline
\end{tabular}

ner Gemeinden verhindern. Die vorgegebenen Präzisionsvorgaben pro Gemeinde, berechnet anhand der Präzisionszielfunktion, werden durch eine geeignete Wahl der Gewichtungsfaktoren $w_{g}(g=1, \ldots, G)$ im resultierenden Optimierungsproblem (11) berücksichtigt.

Zur Erklärung der Funktionsweise des beschriebenen Ansatzes werden in den folgenden Abschnitten ausführliche Analysen präsentiert. Diese basieren meist auf einer synthetischen Grundgesamtheit aller Personen in Deutschland aus dem Jahr 2011. Diese auf Basis eines Registerauszuges erstellte Grundgesamtheit wurde bereits für die Untersuchungen im Rahmen des Stichprobenforschungsprojektes zum Zensus 2011 verwendet (siehe Münnich et al. 2012a). Der Datensatz beinhaltet 11.377 Gemeinden, deren Größenstrukturen in Tab. 1 dargestellt sind. Als Modell für Karteileichen und Fehlbestände wird das sogenannte I1-Modell verwendet. Ausführliche Nachuntersuchungen des Zensus 2011 zeigen, dass dieses Modell im Vergleich zu realen Beobachtung für größere Gemeinden zu geringe Auswirkungen induziert. In kleinen Gemeinden ist die Auswirkung nicht im Detail messbar. Für detailliertere Informationen dazu verweisen wir auf Münnich et al. 2012a. Zudem können Effekte wie Gemeindestrukturreformen, Ausreißer, Schichtwechsler, Nullanschriften und Effekte der Sondermigration nicht adäquat berücksichtigt werden. Zur Schätzung der Varianzreduktionsfaktoren wird Formel (6) auf Basis der Daten der in 2011 registrierten Personen und der in der Zensusstichprobe 2011 erhobenen Personen verwendet. In Kap. 4 wird das Ausmaß der Unsicherheit dieser Schätzungen untersucht, da die Schätzung der Faktoren nur auf einer einzigen Stichprobe beruhen kann.

Die im Folgenden betrachteten Szenarien unterscheiden sich durch die Wahl der folgenden Parameter:

(a) Präzisionszielfunktionen (6 unterschiedliche Bézierkurven, siehe Abb. 2 und 3).

(b) Szenarien zur Wahl der Box-Constraints (8 unterschiedliche Szenarien gebildet aus den unteren Schranken für die Auswahlsätze von $2 \%$ und $5 \%$, sowie den oberen Schranken 50\%, 60\%, $75 \%$ und $100 \%$ ).

(c) Definition von Kleinstgemeinden (6 unterschiedliche Szenarien in Abhängigkeit der Gemeinden, die weniger als eine Mindestzahl an Anschriften beinhalten). In diesen wird eine Vollerhebung durchgeführt (Grenzen 20, 50, 80, 100, 150 und 200).

(d) Verwendung der realen Varianzreduktionsfaktoren beziehungsweise gemittelten Varianzreduktionsfaktoren für BCopt.

(e) Unterschiedliche Aufschlagfaktoren für den aus Amin ermittelten Gesamtstichprobenumfang (von $0 \%$ bis $50 \%$ ).

Zudem beruhen die Ergebnisse bis einschließlich Kap. 3.5 auf einer gemeindescharfen Schichtung, d.h. jede Gemeinde (unabhängig von ihrer Größe) ist als eigene 


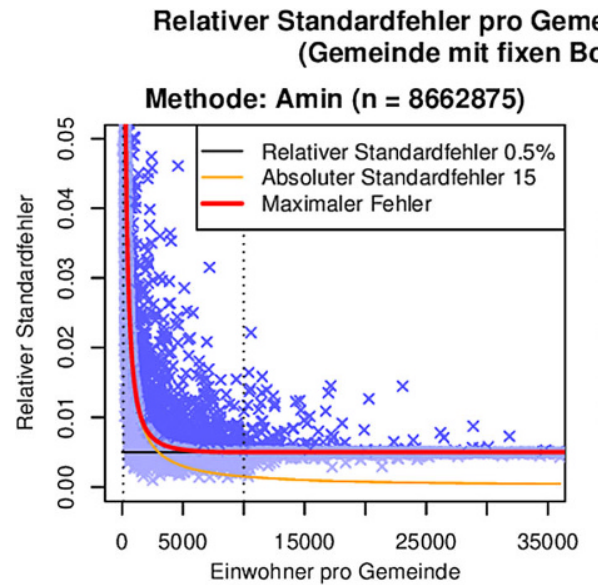

Relativer Standardfehler pro Gemeinde

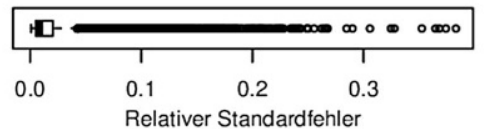

Fehlerhafte Gemeinden: 1141 von 11377

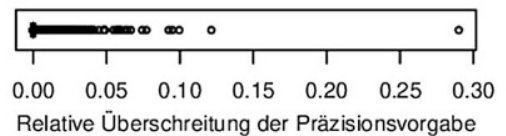

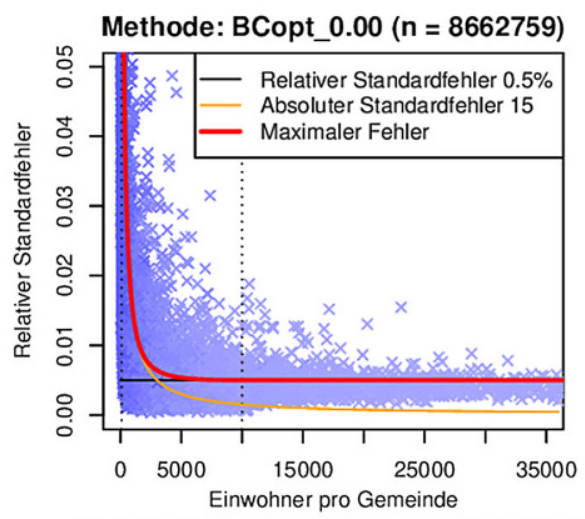

Relativer Standardfehler pro Gemeinde

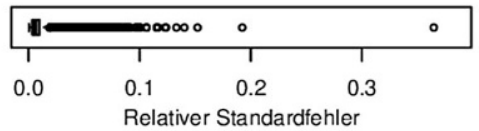

Fehlerhafte Gemeinden: 1616 von 11377

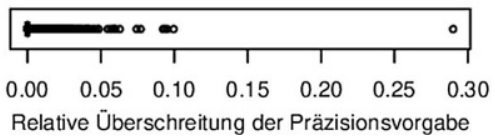

Abb. 4 Relativer Standardfehler bei Bézierkurven (für alle Gemeinden)

Schicht im Design integriert und besitzt eine eigene Präzisionsvorgabe. Aufgrund der heterogenen Größenstruktur der Gemeinden (siehe Tab. 1) wird innerhalb der Gemeinden eine variable Schichtung von 1 bis 16 Schichten verwendet. Im Vergleich dazu wurden 2011 in allen Gemeinden/SMPs eine fixe Zahl von acht Anschriftengrößenklassen verwendet. Durch die Erhöhung der Anschriftengrößenklassen in großen Gemeinden auf bis zu 16 kann eine Reduktion des entsprechenden Stichprobenumfangs bei gleichbleibender Schätzqualität erwartet werden. Eine Verringerung der Anschriftengrößenklassen in kleinen Gemeinden ist notwendig, um aus technischer Sicht eine Stichprobenziehung überhaupt zu ermöglichen. Zudem wird aus technischen Gründen in Kleinstgemeinden bis zu einer festgelegten Größe eine Vollerhebung durchgeführt, da ansonsten die Allokation dort durch Ganzzahligkeitseffekte stark gestört würde.

In Abb. 4 sind die Ergebnisse für ein Szenario in Abhängigkeit des rSE (analog für SE in Abb. 5) dargestellt. Gewählt wird das Szenario mit Bézierkurve bez_1000_15, Vollerhebung in Gemeinden mit weniger als 100 Anschriften, einer unteren Schranke des Auswahlsatzes von $5 \%$ und einer oberen von $50 \%$.

Die Punkte in den oberen Grafiken zeigen jeweils den (theoretisch berechneten) relativen beziehungsweise absoluten Standardfehler einer Gemeinde; je dunkler die Punkte, desto größer ist der entsprechende Auswahlsatz. Die linke Spalte bezieht sich auf die resultierenden Ergebnisse aus Amin. Dort wird also der Gesamtstichpro- 
Absoluter Standardfehler pro Gemeinde mit Funktion: bez_1000_15 (Gemeinde mit fixen Boxen bei $5 \%$ und $50 \%$ )
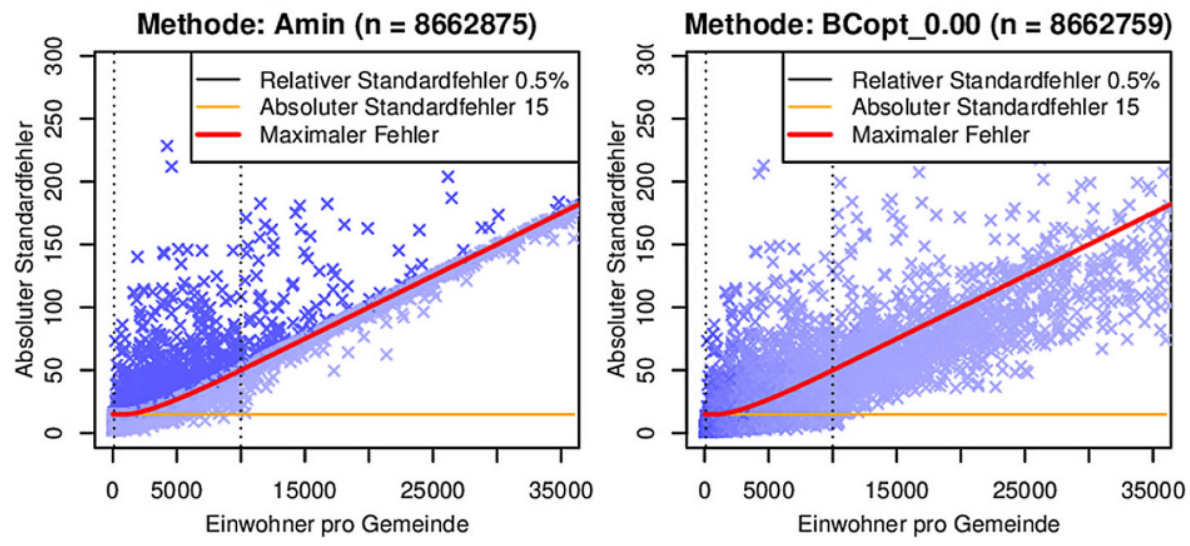

Abb. 5 Absoluter Standardfehler bei Bézierkurven (für alle Gemeinden)

benumfang unter Berücksichtigung der Präzisionszielfunktion und der geschätzten Varianzreduktionsfaktoren festgelegt. Der benötigte Gesamtstichprobenumfang beträgt in diesem Szenario ca. 8,66 Mio. Personen. Die rechte Spalte bezieht sich auf die Ergebnisse der optimalen Allokation mit BCopt, wobei der Gesamtstichprobenumfang aus Amin übernommen wurde. Leichte Differenzen ergeben sich aus der Verwendung der mittleren Einwohnerzahl pro Anschrift $\mathrm{PpA}_{g h}$ in (11). Unter den jeweiligen oberen Plots sind die relativen Standardfehler aller Gemeinden als Boxplots abgetragen (also die entsprechenden y-Werte der darüber liegenden Plots). Hier wird zum einen der kompensatorische Effekt des BCopt deutlich, da bei BCopt eine signifikante Effizienzsteigerung im Vergleich zu Amin zu beobachten ist. Dies ist auch in den oberen Plots erkennbar. Während bei Amin fast alle Punkte in der Nähe der Kurve des maximalen Fehlers liegen, sind bei BCopt vermehrt Punkte deutlich unter der Kurve des maximalen Fehlers zu finden (insbesondere für größere Gemeinden). Diesen Gemeinden wird durch BCopt ein größerer Stichprobenumfang alloziert. Dahingegen sinkt der Stichprobenumfang in den Gemeinden, deren Standardfehler für die geschätzte Einwohnerzahl über der Präzisionszielfunktion liegt. Diese Gemeinden verlieren dabei allerdings nur minimal an Qualität, während andere deutlich an Qualität gewinnen. Zum anderen zeigt der Boxplot, dass der relativen Standardfehler für viele Gemeinden über 0,5\% liegt. Dies ist auf eine Vielzahl von kleinen Gemeinden zurückzuführen (siehe Tab. 1).

Die unteren Boxplots beziehen sich auf die Gemeinden, welche die Präzisionszielfunktion nicht erfüllen, d.h. die Punkte, die über der Kurve des maximalen Fehlers liegen (kurz: fehlerhafte Gemeinden). Neben der Anzahl dieser Gemeinden ist die relative Abweichung von der Präzisionszielfunktion als Boxplot abgetragen. Bei der Verteilung des Gesamtstichprobenumfangs via Amin erfüllen 1.141 Gemeinden, also ca. $10 \%$, nicht die geforderte Präzision. Fast alle dieser Gemeinden sind von der Größe kleiner als 25.000 Einwohner. Die Überschreitung resultiert aus der definierten Obergrenze für die Auswahlsätze pro Schicht von 50\%. Die dunkle Farbe dieser 
Punkte zeigt den höchstmöglichen Auswahlsatz, welcher bei den entsprechenden Gemeinden nicht aus, um die geforderte Präzision einzuhalten. Nach der optimalen und kompensatorischen Allokation mit BCopt steigt die Zahl der Gemeinden mit unzureichender Schätzqualität auf 1.616, was ca. 14.2\% der Gemeinden entspricht. Gleichzeitig steigt allerdings die Qualität bei der Mehrzahl an Gemeinden deutlich an (siehe Boxplots in mittlerer Zeile). Darüber hinaus sinkt der Auswahlsatz für die fehlerhaften Gemeinden teils drastisch, ohne merkliche Qualitätsverluste. Der Grund dafür ist die Schichtung innerhalb der Gemeinden. Die bei weitem größte Varianz geht von der Schicht mit den größten Anschriften aus, d.h. der Auswahlsatz in dieser Schicht ist hauptverantwortlich für die Qualität des Schätzers. Die Schichten mit kleinen Anschriften sind dagegen sehr homogen, d. h. eine Reduktion des Auswahlsatzes reduziert zwar die Qualität, allerdings nicht in einem für die Anwendung bedeutsamen Umfang. Somit spart BCopt in den Schichten mit kleinen Anschriften ein Teil des Stichprobenumfangs ein, um diesen in anderen Gemeinden zur deutlichen Qualitätssteigerung einsetzen zu können. Dies wird als kompensatorischer Effekt bezeichnet und stellt einen der Vorteile der BCopt Allokation dar.

Der Unterschied zwischen den Abb. 4 und 5 liegt ausschließlich in der Betrachtung des absoluten anstatt des relativen Standardfehlers pro Gemeinde.

\subsection{Schätzung der Varianzreduktionsfaktoren}

Wie bereits in Kap. 2 ausführlich erläutert wurde, ist bei der Varianzberechnung des kombinierten Regressionsschätzers (1) pro Gemeinde mittels der Formel (5) die Verwendung des Varianzreduktionsfaktors $\left(1-\vartheta^{2}\right)$, definiert in (6), von entscheidender Bedeutung. Da dieser Faktor im Zensus 2021 zur Berechnung des benötigten Gesamtstichprobenumfangs mittels der Amin-Methode verwendet werden wird, wird dessen Schätzung in diesem Abschnitt erläutert und kritisch analysiert.

Zur Schätzung des Varianzreduktionsfaktors werden die Zensus- und Registerwerte der in der Zensusstichprobe 2011 enthaltenen Anschriften als Hilfsmerkmale verwendet. Dieses Vorgehen kann problematisch sein, da die Schätzungen möglichst nah an den wahren Werten der (unbekannten) Grundgesamtheit liegen sollen, aber nur eine Stichprobe aus dem Jahr 2011 zur Schätzung herangezogen werden kann. Weitere Hilfsmerkmale würden gegebenfalls zur stabileren Schätzung beitragen, liegen aber nicht vor. Die Problematik dieser Schätzung wird in Abb. 6 exemplarisch anhand von drei anonymisierten Gemeinden A, B und C verdeutlicht. Im jeweiligen Scatterplot sind die Registerwerte der Gemeinde gegen die Zensuswerte der Gemeinde aus der Zensusstichprobe 2011 abgetragen. Zudem ist in der Überschrift der berechnete Wert $\vartheta$ (Theta) zur Berechnung des Varianzreduktionsfaktors $\left(1-\vartheta^{2}\right)$ [berechnet durch (6)] und der Korrelationsfaktor $\rho$ (Rho) berechnet durch (7) abgetragen. Zum einen ist zu erkennen, dass $\rho$ und $\vartheta$ deutliche Differenzen aufweisen und somit nicht äquivalent verwendet werden dürfen (vgl. Abschn. 2.2.1). Zum anderen sind hohe Sensitivitäten in den berechneten Werten zu erkennen. In den Gemeinden A und B führen leichte Ausreißer schon zu deutlich reduzierten Werten von $\vartheta$. Im rechten Plot für Gemeinde $C$ ist $\vartheta=0,00$, da ein einziger Ausreißer (siehe untere rechte Ecke) die Berechnung enorm beeinflusst. Diese Sensitivität nimmt bei kleiner werdenden Gemeinden weiter zu. Andere Gemeinden zeigen ähnliche Strukturen. 

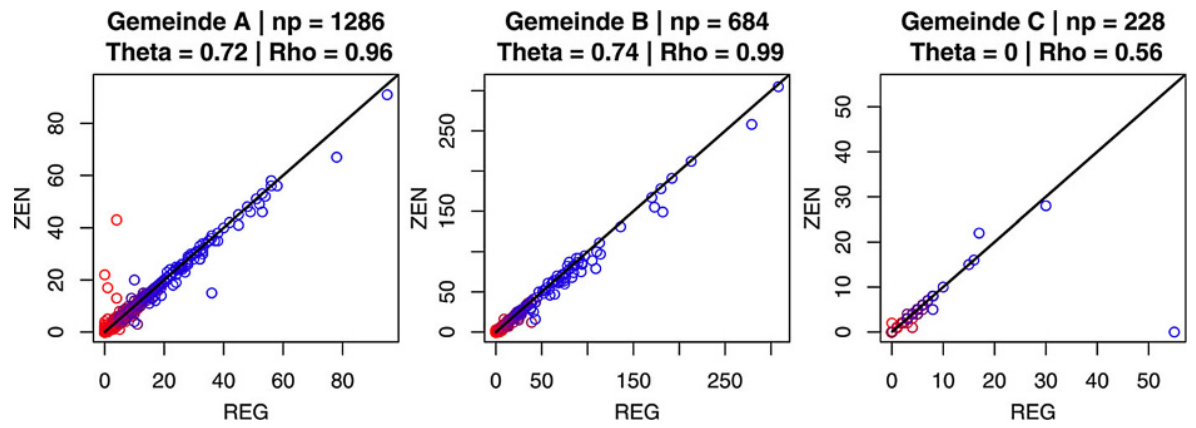

Abb. 6 Vergleich von Register- und Zensuswerten aus Zensusstichprobe 2011 für drei Gemeinden

Um die Sensitivität der Schätzung des Varianzreduktionsfaktors $\left(1-\vartheta^{2}\right)$ detaillierter zu analysieren, wurde eine Monte-Carlo Simulation auf Basis des zu Verfügung stehenden synthetischen Datensatzes aus dem Jahr 2011 durchgeführt. Dabei wurden in 1.000 Replikationen die gemeindespezifischen $\vartheta$ berechnet und deren Verhalten analysiert. Die Ergebnisse sind für 10 ausgewählte Gemeinden als Boxplots in Abb. 7 abgetragen. Jeder Boxplot enthält die gemeindespezifischen Varianzreduktionsfaktoren $\left(1-\vartheta^{2}\right)$ für alle 1.000 Replikationen. Der Mittelwert ist als Linie in jedem Boxplot eingezeichnet. Je größer die Breite der Boxplots, je unsicherer ist die Schätzung des Varianzreduktionsfaktors für diese spezifische Gemeinde. Da die Berechnung auf Basis des synthetischen Datensatzes unter Berücksichtigung spezieller Modelle von Karteileichen und Fehlbeständen erfolgt, kann aus der absoluten Höhe der Varianzreduktionsfaktoren in dieser Abbildung kein Rückschluss auf die Realität gezogen werden; lediglich die Verteilung dieser über die 1.000 Replikationen ist von Relevanz.

Abb. 7 Verteilung der Reduktionsfaktoren bei 1.000 Durchläufen (auf Basis des Simulationsdatensatzes).

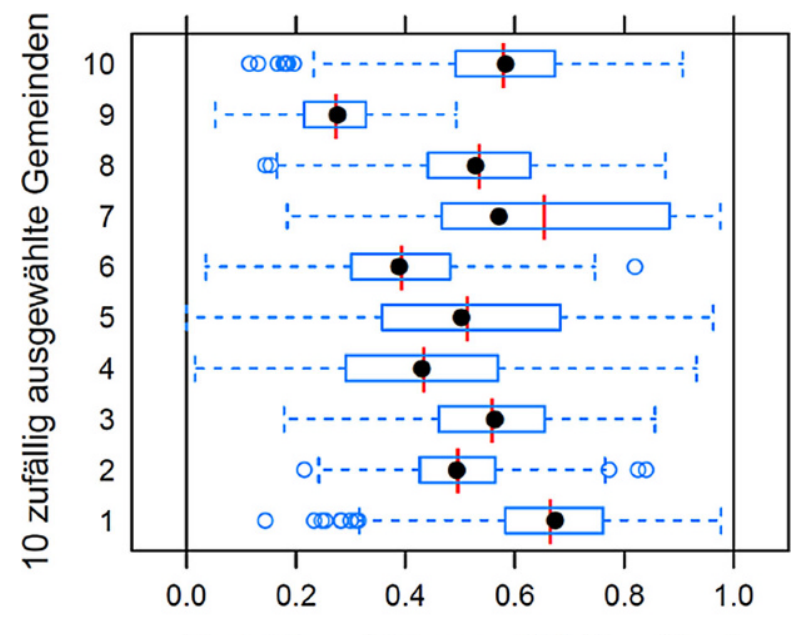

Reduktionsfaktor pro MC-Durchlauf 
Abb. 8 Verteilung der Reduktionsfaktoren (geschätzt aus der Zensusstichprobe 2011) auf die Bundesländer.

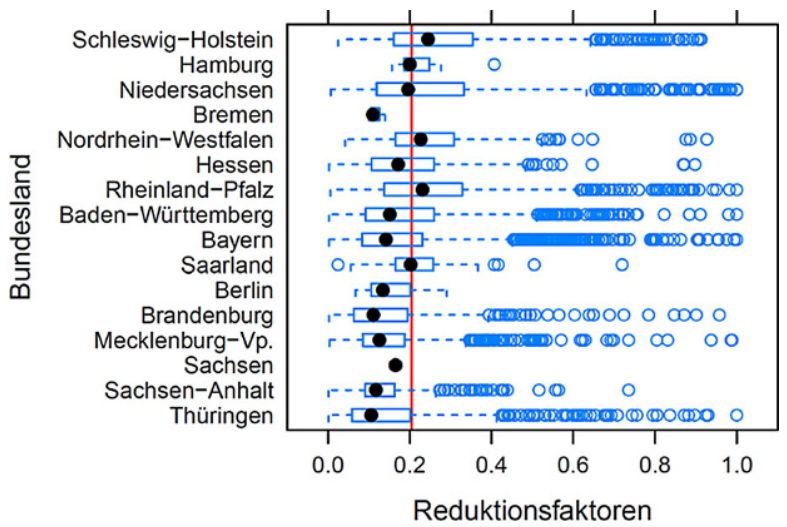

Für alle der 10 zufällig ausgewählten Gemeinden weist die Verteilung der Varianzreduktionsfaktoren über die 1.000 Replikationen eine sehr hohe Varianz auf. Dies verdeutlicht die Problematik der Schätzung der Reduktionsfaktoren anhand der einzigen verfügbaren Stichprobe aus dem Zensus 2011. Es ist somit eine hohe Unsicherheit in diesen Schätzungen vorhanden, die es bei der Berechnung eines benötigten Gesamtstichprobenumfangs mit Amin zu berücksichtigen gilt. Zwei alternative Ansätze dafür werden in Kap. 4 beschrieben. Weitere Untersuchungen zeigen jedoch, dass über alle Gemeinden hinweg der gemittelte Varianzreduktionsfaktor einigermaßen stabil geschätzt werden kann. Somit wird ein Teil der Unsicherheit gedämpft, da sich zu hohe Schätzungen mit zu niedrigen ausgleichen. Da die letztendliche Allokation mit BCopt nur die gemittelten Varianzreduktionsfaktoren verwendet (vgl. Abschn. 3.3), haben die ungenauen Schätzungen der $\vartheta$ keinen Einfluss auf die Allokation, wohl aber auf den Gesamtstichprobenumfang.

Die geschätzten Varianzreduktionsfaktoren aus der Zensusstichprobe 2011 weisen neben der Unsicherheit eine Heterogenität in Bezug auf die Länder aus. Dies wird in Abb. 8 verdeutlicht, in welcher die geschätzten Varianzreduktionsfaktoren auf Basis der Zensusstichprobe 2011 in Abhängigkeit des Bundeslandes aufgelistet sind. Dabei ist auffällig, dass Länder mit eher kleinen Gemeinden (bspw. Schleswig-Holstein, Rheinland-Pfalz und Thüringen) tendenziell eher höhere Varianzreduktionsfaktoren haben, was einen geringeren Effizienzgewinn von der gebundenen Hochrechnung im Vergleich zur freien Hochrechnung impliziert.

Neben der instabilen Schätzung des Varianzreduktionsfaktors ist die theoretische Fundierung seiner Verwendung zu diskutieren. Da der Varianzreduktionsfaktor aus der Zensusstichprobe des Jahres 2011 geschätzt werden muss, basiert dieser auf möglicherweise veralteten Daten. Zudem impliziert die Verwendung von gemeindespezifischen Varianzreduktionsfaktoren aus dem Jahr 2011 eine mögliche Ungleichbehandlung von Gemeinden im Jahr 2021. Daher wird der Varianzreduktionsfaktor ausschließlich zur Schätzung des benötigten Gesamtstichprobenumfangs mittels Amin verwendet und nicht für BCopt (siehe Abschn. 2.2.2). Im Hinblick darauf zeigt Abb. 9 die resultierenden relativen Standardfehler pro Gemeinde (in logarithmischer Skala) im Vergleich. Auf der x-Achse sind die Werte berechnet mit der BCopt-Allokation und den geschätzten Reduktionsfaktoren abgetragen, auf der 
Abb. 9 Scatterplot (log-Skala) über die Auswirkung der aus der Zensusstichprobe 2011 geschätzten Reduktionsfaktoren auf BCopt (verglichen mit dem Fall konstanter Reduktionsfaktoren).

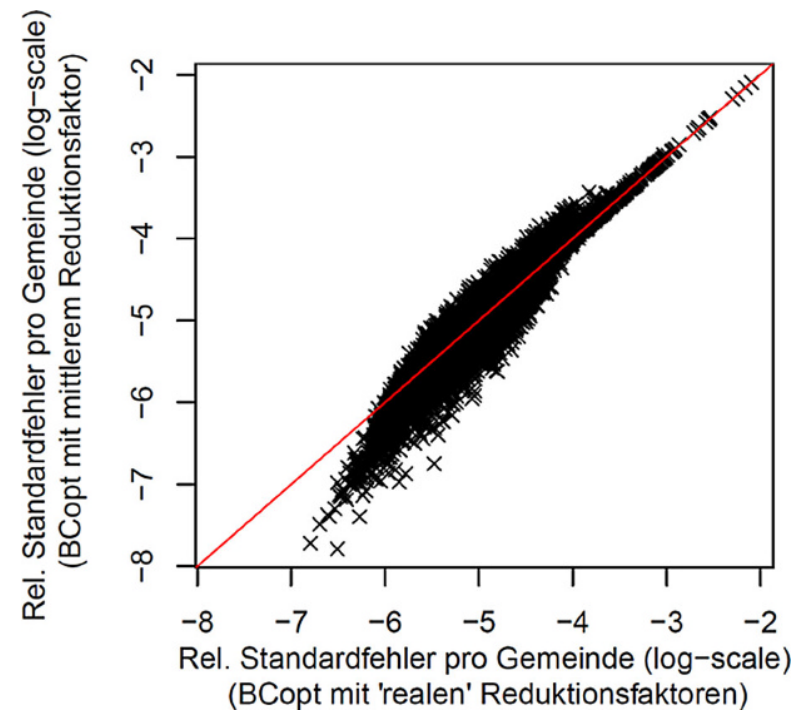

y-Achse die entsprechenden Werte unter Verwendung der konstanten Varianzreduktionsfaktoren für alle Gemeinden (tatsächliche Vorgehen im Zensus 2021). Generell sind ähnliche relative Standardfehler zu beobachten, wobei die Schätzqualität in Gemeinden mit sehr hohen Schätzqualitäten durch den Verzicht der Verwendung der Varianzreduktionsfaktoren noch weiter verbessert werden kann. Der Spread zwischen den Schätzqualitäten steigt also bei der Verwendung von konstanter Varianzreduktionsfaktoren an. Der Grund dafür ist, dass Gemeinden mit hohen Schätzqualitäten i.d.R. größere und in der Anschriftenstruktur homogenere Gemeinden sind. Diese haben meist auch höhere Varianzreduktionsfaktoren, was bei der Allokation mit BCopt unter Verwendung der Faktoren zu einer Untergewichtung und somit geringerem Stichprobenumfang führt. Diese Untergewichtung wird bei konstanten Reduktionsfaktoren vermieden, sodass ein größerer Stichprobenumfang dieser Gemeinde alloziert wird. Damit steigt die ohnehin schon gute Schätzqualität weiter an.

\subsection{Festlegungen der Nebenbedingungen}

Neben der bereits ausführlich diskutierten Präzisionszielfunktion werden im Folgenden die Auswirkungen von verschiedenen Definitionen der Box-Constraints, also der unteren und oberen Grenzen für die Auswahlsätze diskutiert. Generell führt eine höhere untere Grenze dazu, dass die Auswahlsätze über die Gemeinden und Schichten homogener verteilt werden. Eine Unterrepräsentation einzelner Gemeinden oder Schichten wird damit ausgeschlossen, was insbesondere im Hinblick auf die späteren Ziel-2-Schätzungen von großer Bedeutung sein kann. Die Festlegung der oberen Schranken hat jedoch größere Auswirkungen, sodass die Wahl dieser Schranken einer kritischen Diskussion bedarf. Ein Grund dafür ist das gewählte Stichprobendesign und insbesondere die Schichtung innerhalb jeder Gemeinde 
Tab. 2 Gesamtstichprobenumfang (Anzahl der Personen) in Abhängigkeit von Kleinstgemeinden und Box-Constraints und Präzisionszielfunktion bez_1000_25.

\begin{tabular}{lrrrrrr}
\hline & ADR_20 & ADR_50 & ADR_80 & ADR_100 & ADR_150 & ADR_200 \\
\hline const_5_50 & 8.018 .318 & 8.049 .313 & 8.138 .541 & 8.223 .971 & 8.500 .735 & 8.872 .439 \\
const_5_60 & 7.546 .903 & 7.579 .078 & 7.667 .877 & 7.751 .948 & 8.028 .527 & 8.400 .203 \\
const_5_75 & 6.928 .830 & 6.959 .339 & 7.048 .765 & 7.133 .753 & 7.411 .025 & 7.782 .803 \\
const_5_100 & 6.072 .883 & 6.103 .709 & 6.192 .792 & 6.278 .113 & 6.556 .222 & 6.929 .674 \\
const_2_50 & 6.416 .632 & 6.448 .641 & 6.539 .226 & 6.626 .125 & 6.909 .712 & 7.290 .681 \\
const_2_60 & 5.945 .799 & 5.977 .799 & 6.067 .777 & 6.154 .731 & 6.438 .137 & 6.818 .240 \\
const_2_75 & 5.342 .256 & 5.373 .813 & 5.465 .473 & 5.553 .139 & 5.836 .329 & 6.217 .990 \\
const_2_100 & 4.524 .112 & 4.555 .908 & 4.646 .073 & 4.733 .255 & 5.016 .371 & 5.398 .904 \\
\hline
\end{tabular}

nach Anschriftengrößen. Da die Varianz der Anschriftengröße in Schichten mit großen Anschriften von Natur aus größer ist, sind diese Schichten bei der Allokation von entscheidender Bedeutung. Daher liegt der Auswahlsatz in diesen Schichten deutlich über dem Durchschnitt. Dies führt zum einen zu einer heterogenen Verteilung der Auswahlsätze, wodurch unter anderem die Schätzung der Ziel-2-Variablen deutlich leiden wird und zum anderen zu einer deutlichen Ungleichverteilung des Befragungsaufwandes. Zur Vermeidung dieser Probleme kann die obere Schranke heruntergesetzt werden.

Neben diesen Aspekten hat die Definition der Schranken einen Einfluss auf den benötigten Gesamtstichprobenumfang, der sich aus Amin ergibt. Die Tab. 2 zeigt benötigte Gesamtstichprobenumfänge in Abhängigkeit der gewählten Box-Constraints und der Definition der Kleinstgemeinden (Ergebnis von Amin). Dabei steht z. B. ADR_50 für eine Vollerhebung in allen Gemeinden mit weniger als 50 Anschriften. Die Bezeichnung const_5_75 repräsentiert die Grenzen für einen unteren Auswahlsatz von $5 \%$ und einen oberen von $75 \%$. Auffällig ist, dass die Stichprobenumfänge in Bezug auf die Definition von Kleinstgemeinden nicht erheblich ansteigen, beispielsweise steigt der Gesamtstichprobenumfang bei der Definition der Box-Constraints von 5\% und 50\% (erste Zeile) nur um ca. 2,17\% an, falls die Kleinstgemeinden als Gemeinden kleiner 100 statt kleiner 50 Anschriften definiert werden. Es ist jedoch zu beachten, dass diese Ergebnisse auf einer synthetisch erzeugten Grundgesamtheit basieren und in der Realität abweichen können.

Vergleicht man die unterschiedlichen Szenarien der Box-Constraints, so sind enorme Unterschiede festzustellen. Wir unterscheiden zwischen Kombinationen aus den unteren Schranken von 2\% und 5\%, sowie den oberen Schranken von 50\%, 60\%, $75 \%$ und $100 \%$. Bei einer unteren Schranke von $2 \%$ liegt der Stichprobenumfang um ca. $20 \%$ unter dem Stichprobenumfang bei einer unteren Schranke von $5 \%$. Konträr der normalen Intuition sinkt allerdings der Stichprobenumfang mit einer Erhöhung der oberen Schranke teilweise deutlich ab. Der Hauptgrund dafür ist erneut die Schichteinteilung nach Anschriftengrößen. Bei einer oberen Box von 50\% wird bei Amin der Stichprobenumfang zuerst in der obersten Schicht voll ausgenutzt ohne die geforderte Präzision einzuhalten, da im Allgemeinen diese oberste Schicht die höchste Schichtvarianz aufweist. Danach werden sukzessive mehr Einheiten aus den kleineren Schichten gezogen (ohne große Verbesserungen der Qualität), sodass 
Tab. 3 Auswahlsätze pro Schicht (S1-S7) für eine ausgewählte Gemeinde (mit ca. 1.500 Einwohnern) mit sieben Schichten in Abhängigkeit von oberen Box-Constraints in Allokationsmethode.

\begin{tabular}{lrrrrrrrr}
\hline & $\mathrm{S} 1$ & $\mathrm{~S} 2$ & $\mathrm{~S} 3$ & $\mathrm{~S} 4$ & $\mathrm{~S} 5$ & $\mathrm{~S} 6$ & $\mathrm{~S} 7$ & $\mathrm{rSE}$ \\
\hline $\begin{array}{l}\text { Amin: } \\
\text { const_5_50 }\end{array}$ & $\mathbf{5 0 \%}$ & $\mathbf{5 0 \%}$ & $\mathbf{5 0} \%$ & $\mathbf{5 0} \%$ & $\mathbf{5 0} \%$ & $\mathbf{5 0} \%$ & $\mathbf{5 0} \%$ & $3,18 \%$ \\
$\begin{array}{l}\text { Amin: } \\
\text { const_5_60 }\end{array}$ & $60 \%$ & $60 \%$ & $60 \%$ & $60 \%$ & $60 \%$ & $60 \%$ & $\mathbf{6 0} \%$ & $2,80 \%$ \\
$\begin{array}{l}\text { Amin: } \\
\text { const_5_75 }\end{array}$ & $75 \%$ & $75 \%$ & $75 \%$ & $75 \%$ & $75 \%$ & $75 \%$ & $\mathbf{7 5 \%}$ & $1,83 \%$ \\
$\begin{array}{l}\text { Amin: } \\
\text { const_5_100 }\end{array}$ & $\mathbf{1 0 \%}$ & $\mathbf{6 \%}$ & $\mathbf{6 \%}$ & $\mathbf{9 \%}$ & $\mathbf{6 \%}$ & $\mathbf{1 1 \%}$ & $\mathbf{1 0 0 \%}$ & $1,72 \%$ \\
$\begin{array}{l}\text { BCopt: } \\
\text { const_5_50 }\end{array}$ & $32 \%$ & $17 \%$ & $4 \%$ & $18 \%$ & $6 \%$ & $18 \%$ & $\mathbf{5 0 \%}$ & $3,29 \%$ \\
$\begin{array}{l}\text { BCopt: } \\
\text { const_5_60 }\end{array}$ & $26 \%$ & $14 \%$ & $4 \%$ & $14 \%$ & $6 \%$ & $16 \%$ & $\mathbf{6 0} \%$ & $2,88 \%$ \\
$\begin{array}{l}\text { BCopt: } \\
\text { const_5_75 }\end{array}$ & $21 \%$ & $10 \%$ & $4 \%$ & $12 \%$ & $6 \%$ & $13 \%$ & $\mathbf{7 5 \%}$ & $2,22 \%$ \\
$\begin{array}{l}\text { BCopt: } \\
\text { const_5_100 }\end{array}$ & $15 \%$ & $7 \%$ & $4 \%$ & $9 \%$ & $6 \%$ & $8 \%$ & $\mathbf{1 0 0} \%$ & $1,59 \%$ \\
\hline
\end{tabular}

letztendlich ein Auswahlsatz von 50\% in jeder Schicht der Gemeinde gegeben ist. Dies ist in Zeile 1 der Tab. 3 beispielhaft für eine Gemeinde mit sieben Schichten und ca. 1.500 Einwohnern abgebildet, welche die Präzisionsvorgabe nicht erfüllt. In den Spalten S1 bis S7 sind die jeweiligen schichtspezifischen Auswahlsätze abgetragen. Ein ähnliches Verhalten ist bei oberen Boxen von $60 \%$ und $75 \%$ zu erkennen. Wird nun allerdings die obere Box auf $100 \%$ gesetzt, so reicht ein deutlich geringerer Stichprobenumfang bei gleichzeitiger Vollerhebung in der höchsten Schicht aus, um die Qualität einzuhalten. Dies erklärt, warum sich der Stichprobenumfang bei steigenden oberen Box-Constraints deutlich absinkt. Dieses Phänomen tritt in besonderer Form in den Gemeinden auf, die das Präzisionsziel nicht einhalten würden. Die resultierenden Auswahlsätze nach der Verteilung des berechneten Stichprobenumfangs mit BCopt sind im zweiten Teil der Tabelle abzulesen. Diese unterscheiden sich deutlich von den mit Amin berechneten Werten. Die Gründe hierfür wurden bereits in Abschn. 3.3 erläutert. Der Stichprobenumfang wird so umgeschichtet, dass die Reduktion der Varianz des Populationsschätzers maximal ist. Trotz einer deutlichen Verringerung des Auswahlsatzes in dieser einen Gemeinde steigt der relative Standardfehler nur minimal an.

Nach Analyse der Tab. 2 und 3 kann also aufgrund der Reduzierung des Gesamtstichprobenumfangs und der Vermeidung eines über alle Schichten hinweg konstant hohen Auswahlsatzes eine obere Schranke von $100 \%$ als vorteilhaft angesehen werden. Allerdings ergeben sich nicht zu vernachlässigende Nachteile, die es abzuwägen gilt. Abbildung 10 zeigt für jede Gemeinde die Verteilung der Auswahlsätze der obersten Schicht in Abhängigkeit von der Wahl der Box-Constraints nach der BCopt Allokation. Die oberen Kurven kennzeichnen die oberste Schicht, die unteren Kurven stellen den durchschnittlichen Auswahlsatz der Gemeinde über alle Schichten dar. Die durchgezogene Linie entspricht dem Median; die gestrichelten Linien zeigen die 5\%, 25\%, 75\% und 95\% Quantile. Maxima und Minima sind als 


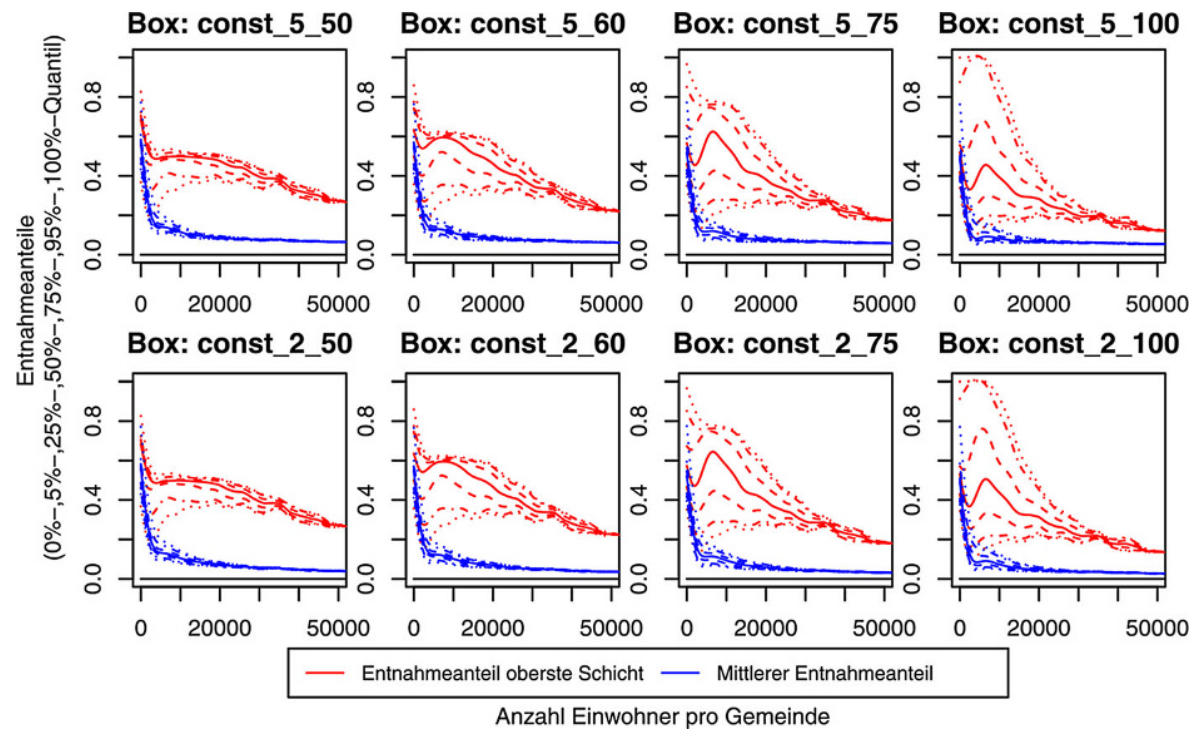

Abb. 10 Auswahlsätze für unterschiedliche Szenarien von Box-Constraints (mittlere Auswahlsätze pro Gemeinde versus Auswahlsätze der obersten Schicht) nach der BCopt Allokation

gepunktete Linien eingezeichnet. Bei den Szenarien mit oberer Schranke von 100\% (rechte Plots) erkennt man insbesondere für Gemeindegrößen bis ca. 15.000 Einwohnern extreme Verteilungen für die oberste Schicht. In diesen Gemeinden findet oft eine Vollerhebung statt. Die mittleren Auswahlsätze liegen deutlich niedriger. Somit ergeben sich durch eine obere Box von $100 \%$ sehr heterogene Auswahlsätze innerhalb der Gemeinden. Wird die obere Box heruntergesetzt, so wird dieser Effekt abgeschwächt. Die oberen und unteren Kurven nähern sich an, obwohl der Auswahlsatz für große Anschriften immer noch deutlich über dem Durchschnitt liegt.

Bei der Entscheidung für eine Wahl der unteren Grenze des Auswahlsatzes zwischen 2\% und 5\% spielen mehrere Faktoren eine Rolle. Wie schon erwähnt, verhindert ein minimaler Auswahlsatz von 5\% eine extreme Ungleichverteilung der Auswahlsätze über die Gemeinden und Schichten. Dies ist zum einen für die Ziel-2Schätzungen vorteilhaft, wirkt sich jedoch auch positiv auf die Einwohnerzahlermittlung aus. Ein minimaler Auswahlsatz von 5\% führt zu sinkenden Designgewichten in der Schicht, verringert also die negative Auswirkung von Anschriften auf die Schätzung, die einer falschen Schicht zugeordnet wurden (z.B. Schichtwechsler, Nullanschriften, Karteileichen und Fehlbestände). Zudem dämpft sie etwaig auftretende Problematiken in den Nachuntersuchungen ab.

Vergleicht man nun die bundeslandspezifischen Auswahlsätze, so ergeben sich deutliche Unterschiede zwischen den Bundesländern. Der Hauptgrund dafür ist die teils sehr unterschiedliche Gemeindestruktur in den Bundesländern. Beispielsweise beinhaltet Nordrhein-Westfalen ungefähr 400 Gemeinden bei zirka 18 Mio. Einwohnern. Dahingegen hat Rheinland-Pfalz bei einer Einwohnerzahl von ca. 4 Mio. ungefähr 2.300 Gemeinden. Diese Vielzahl (kleiner) Gemeinden impliziert höhe- 
Tab. 4 Auswahlsätze (Personen) pro Bundesland in Abhängigkeit von Wahl der Box-Constraints für ADR_100 und Präzisionszielfunktion bez_1000_25.

\begin{tabular}{lrrrrrr}
\hline & const_5_50 & const_5_75 & const_5_100 & const_2_50 & const_2_75 & const_2_100 \\
\hline SH & $14,0 \%$ & $12,1 \%$ & $10,4 \%$ & $12,6 \%$ & $10,8 \%$ & $9,3 \%$ \\
HH & $5,3 \%$ & $5,1 \%$ & $5,1 \%$ & $2,5 \%$ & $2,3 \%$ & $2,2 \%$ \\
NI & $9,4 \%$ & $8,1 \%$ & $7,1 \%$ & $7,5 \%$ & $6,1 \%$ & $5,2 \%$ \\
HB & $5,3 \%$ & $5,1 \%$ & $5,0 \%$ & $2,5 \%$ & $2,3 \%$ & $2,2 \%$ \\
NW & $6,4 \%$ & $5,9 \%$ & $5,6 \%$ & $3,9 \%$ & $3,4 \%$ & $3,0 \%$ \\
HE & $9,0 \%$ & $7,9 \%$ & $7,0 \%$ & $7,3 \%$ & $6,0 \%$ & $5,1 \%$ \\
RP & $16,2 \%$ & $13,5 \%$ & $11,6 \%$ & $14,8 \%$ & $12,4 \%$ & $10,8 \%$ \\
BW & $10,3 \%$ & $8,9 \%$ & $7,6 \%$ & $8,7 \%$ & $7,3 \%$ & $6,1 \%$ \\
BY & $11,4 \%$ & $9,6 \%$ & $8,1 \%$ & $9,9 \%$ & $8,2 \%$ & $6,8 \%$ \\
SL & $7,8 \%$ & $7,0 \%$ & $6,4 \%$ & $5,7 \%$ & $4,8 \%$ & $4,1 \%$ \\
BE & $5,2 \%$ & $5,0 \%$ & $5,0 \%$ & $2,4 \%$ & $2,2 \%$ & $2,1 \%$ \\
BB & $11,0 \%$ & $9,2 \%$ & $7,8 \%$ & $9,5 \%$ & $7,7 \%$ & $6,3 \%$ \\
MV & $16,4 \%$ & $13,8 \%$ & $11,5 \%$ & $15,2 \%$ & $12,8 \%$ & $10,8 \%$ \\
SN & $10,4 \%$ & $9,0 \%$ & $7,8 \%$ & $8,8 \%$ & $7,4 \%$ & $6,2 \%$ \\
ST & $9,2 \%$ & $8,0 \%$ & $7,0 \%$ & $7,3 \%$ & $6,0 \%$ & $5,1 \%$ \\
TH & $15,3 \%$ & $13,0 \%$ & $11,1 \%$ & $13,9 \%$ & $11,8 \%$ & $10,1 \%$ \\
DE & $10,24 \%$ & $8,88 \%$ & $7,81 \%$ & $8,25 \%$ & $6,91 \%$ & $5,89 \%$ \\
\hline
\end{tabular}

re Auswahlsätze, die zur Einhaltung der vorgegebenen Präzision notwendig sind. Dementsprechend hat Rheinland-Pfalz bei Box-Constraints von $5 \%$ und $50 \%$ einen mittleren Auswahlsatz von 16,2\%, wohingegen Nordrhein-Westfalen mit 6,4\% auskommt. Diese Werte sind in der folgenden Tab. 4 abzulesen. Eine weitere Auffälligkeit sind die sehr geringen Auswahlsätze in den Stadtstaaten Berlin, Hamburg und Bremen, da diese sehr homogene Anschriftenstrukturen aufweisen. Des Weiteren ist bei diesen drei Ländern kein unmittelbar erkennbarer Unterschied zwischen den Szenarien mit oberer Grenze der Auswahlsätze von 50\%, 75\% und $100 \%$ $\mathrm{zu}$ erkennen. Zudem spielen die Varianzreduktionsfaktoren, definiert in (6), und etwaige Aufschlagfaktoren eine entscheidende Rolle. Deshalb ist die Höhe des Gesamtauswahlsatzes nicht Eins zu Eins in die Realität übertragbar, insbesondere weil die Berechnungen auf dem synthetischen Datensatz basieren. Für eine ausführliche Analyse über die Bestimmung des Gesamtstichprobenumfangs sei auf Kap. 4 verwiesen.

\subsection{Alternative Ansätze für kleine Gemeinden und Verbandsgemeinden}

Die in den Kap. 3.3 bis 3.5 präsentierten Ergebnisse basieren ausschließlich auf einer regionalen Stratifizierung nach Gemeinden. Als Konsequenz ergeben sich sehr viele kleine Gemeinden (siehe Tab. 1). Zum Beispiel gibt es über 1.000 Gemeinden, die weniger als 100 Anschriften beinhalten. Sollen also in diesen kleinen Gemeinden statistische Auswertungen erstellt werden, so ist die Diskussion über die Sinnhaftigkeit dieser Auswertungen kritisch zu erörtern. In dem Zusammenhang ist eine Definition von sogenannten Kleinstgemeinden, in denen eine Vollerhebung durchgeführt werden muss, fast unumgänglich. Dazu wurden in Kap. 3.5 bereits Auswertungen 



Abb. 11 Überdeckungsraten in Abhängigkeit der Gemeindegröße (Szenario mit Boxen bei $5 \%$ und 50\%, sowie Vollerhebung in Kleinstgemeinden mit weniger als 100 Anschriften).

gezeigt. So ließen sich zwar gemeindespezifische Punkt- und Varianzschätzungen für alle Gemeinden technisch durchführen, allerdings mit der Folge, dass in kleinen Gemeinden jede Anschrift zu befragen ist. In den Kap. 3.3 bis 3.5 wurden Vollerhebungen in der Regel in Gemeinden kleiner als 100 Anschriften durchgeführt.

In Abb. 11 sind für diesen beschriebenen Fall die Konfidenzintervallüberdeckungsraten des $95 \%$ Konfidenzintervalls für die gemeindespezifischen Schätzungen dargestellt. Die Überdeckungsraten sind dabei in Relation zur Gemeindegröße abgetragen. Während der linke Plot die Abhängigkeit von der Einwohnerzahl der Gemeinde darstellt, bildet der rechte Plot die Überdeckungsrate in Relation zur Anzahl der Anschriften innerhalb der Gemeinde ab. $\mathrm{Zu}$ beachten ist dabei, dass die $\mathrm{x}$-Achse jeweils als log-Skala abgebildet ist. Da in Gemeinden mit weniger als 100 Anschriften eine Vollerhebung durchgeführt wird, sind links von der vertikalen Linie im rechten Plot die Überdeckungsraten gleich $100 \%$. Darüber hinaus wird deutlich, dass insbesondere bei Gemeinden mit weniger als 2.000 Einwohnern (vertikale Linie im linken Plot) beziehungsweise mit weniger als 200 Anschriften die Überdeckungsraten problematisch sind, so dass eine Vollerhebung in Gemeinden kleiner als 100 Anschriften nicht ausreicht, um die Problematik der instabilen Schätzungen zu lösen. Im Gegensatz dazu lässt sich aus dem Urteil des Bundesverfassungsgerichtes (siehe BVerfG 2018) ableiten, dass eine Vollerhebung unter anderem aufgrund der informationellen Selbstbestimmung der Bürger (falls möglich) vermieden werden soll.

Im Folgenden werden drei Alternativen aufgezeigt, die der Diskussion beim Bundesverfassungsgericht gerecht werden, gemeindespezifische Auswertungen zulassen, aber auf die explizite Definition von Kleinstgemeinden und somit auf eine Vollerhebung in kleinen Gemeinden weitestgehend verzichten. Dazu werden die Präzisionsanforderung nicht mehr auf der regionalen Ebene der Gemeinden definiert, sondern für neu gebildete SMPs über Berücksichtigung von Gemeindeverbänden. Im Folgenden werden die Box-Constraints, also der minimal und maximal zulässige Auswahlsatz, auf $5 \%$ und $50 \%$ fixiert.

Die erste Möglichkeit zur Vermeidung der expliziten Definition von Kleinstgemeinden (bezeichnet mit GEM_100; Alternative A) basiert auf der ursprünglichen 
Schichtung nach Gemeinden. Um stabile Regressionsschätzungen auch in den kleinen Gemeinden zu gewährleisten, wird eine Mindeststichprobengröße pro Gemeinde von 100 Anschriften festgesetzt. Die theoretische Fundierung dieser Grenze basiert zum einen auf der Instabilität von Regressionsschätzungen in kleineren Regionen, zum anderen auf den analysierten Konfidenzintervallüberdeckungsraten im Fall der Vollerhebungen bei Kleinstgemeinden in Abb. 11. Somit wird ab einer Gemeindegröße von 200 Einwohnern die obere Grenze des Auswahlsatzes von 50\% so angepasst, dass eine Mindeststichprobengröße von 100 Anschriften zulässig ist. Zudem muss die untere Grenze so angepasst werden, dass die Mindestzahl von 100 Anschriften nicht unterschritten wird.

Grundlage des zweiten Konzeptes (bezeichnet mit VBG_Reste; Alternative B) ist die Definition von Verbandsgemeinden und eine damit einhergehende veränderte Schichtungsmethodik. Verbandsgemeinden stimmen in vielen Bundesländern und für große Gemeinden mit den Gemeinden überein, mehrere kleinere Gemeinden werden allerdings zu einer Verbandsgemeinde zusammengefasst. Allerdings soll nicht ausschließlich nach Verbandsgemeinden geschichtet werden, sondern nur kleine Gemeinden zusammengefasst werden. Dazu wird folgende Schichtung für die Alternative B vorgeschlagen:

(i) Gemeinden mit mehr als 2.000 Einwohnern bilden eine eigene Schicht (unverändert zu Basisvariante und Alternative A).

(ii) Gemeinden mit weniger als 2.000 Einwohnern werden zusammengefasst, sofern sie innerhalb einer Verbandsgemeinde liegen.

Die Grenze von 2.000 Einwohnern basiert auf der Analyse der Konfidenzintervallüberdeckungsraten in Abb. 11 sowie weiterer ausführlicher Untersuchungen zu der resultierenden Größenstruktur der SMPs bei der Wahl anderer Grenzen. Die Schichtung innerhalb einer Gemeinde bleibt unverändert variabel mit einer Anzahl von 1 bis 16 Schichten. Somit ergeben sich 6.518 sogenannte SMPs (statt 11.377 Gemeinden). Von diesen SMPs bilden 5.395 Stück ganze Gemeinden über 2.000 Einwohnern ab; die restlichen 1.123 SMPs sind somit Zusammenschlüsse der übrigen 5.982 Gemeinden. Als Folge sinkt die Zahl von Gemeinden/SMPs mit weniger als 100 Anschriften von über 1.000 auf zwei. Somit sind statistische Auswertungen mittels des kombinierten Regressionsschätzers und dessen Varianzschätzung für nahezu alle neu gebildeten SMPs möglich.

Das dritte Konzept beim Umgang mit Kleinstgemeinden (bezeichnet mit VBG; Alternative C) ähnelt der zweiten Möglichkeit, allerdings werden die Gemeinden ab 2.000 Einwohnern nicht mehr separat betrachtet; sie verbleiben in der zugehörigen Verbandsgemeinde. Letztendlich wird die Einhaltung des Präzisionsziels bei dieser Variante also nur auf Verbandsgemeindeebene gefordert. Aus Alternative C ergeben sich 4.573 SMPs, die deckungsgleich mit den Verbandsgemeinden sind. Somit ist die Durchführung statistischer Auswertungen mittels des kombinierten Regressionsschätzers und dessen Varianzschätzung bei Alternative $\mathrm{C}$ noch weniger problematisch als bei Alternative B.

Der Vergleich der Größenordnungen der entstandenen SMPs sowie der Verbandsgemeinden (VBG) verglichen mit den Größen aller Gemeinden ist in Tab. 5 abgebildet. Als Folge der Zusammenfassung von mehreren Gemeinden zu SMPs bei 
Tab. 5 Quantile der SMP-Größen aller 6.518 SMPs, 4.573 Verbandsgemeinden und 11.377 Gemeinden

\begin{tabular}{lrrrrrr}
\hline Alternative & Anzahl & Quantile & & & & \\
& & $0 \%$ & $25 \%$ & $50 \%$ & $75 \%$ & $100 \%$ \\
\hline $\begin{array}{l}\text { A: Gemeindegröße } \\
\text { (GEM_100) }\end{array}$ & 11.377 & 9 & 644 & 1.692 & 5.139 & 365.598 \\
B: SMP-Größe (VBG_Reste) & 6.518 & 97 & 2.855 & 4.967 & 10.238 & 365.598 \\
C: Verbandsgemeindegröße & 4.573 & 368 & 4.972 & 8.931 & 15.704 & 365.598 \\
(VBG) & & & & & & \\
\hline
\end{tabular}

den Alternativen B und C werden nicht mehr alle Gemeinden als explizite Schicht im Stichprobendesign repräsentiert, da die Schichtung auf Basis der SMPs gebildet wird. Dies betrifft bei Alternative B insbesondere die kleinen Gemeinden. Somit ist der Stichprobenumfang innerhalb der Gemeinden, die Teil eines SMPs sind, a priori unbekannt. Im Extremfall kann es vorkommen, dass einer Gemeinde keine Stichprobeneinheit zugeordnet wird. Um gleichzeitig Ergebnisse für die kleinen Gemeinden auszuweisen, empfehlen wir synthetische Schätzmethoden beziehungsweise modellassistierte Schätzungen mittels eines verallgemeinerten Kalibrierungsschätzers (vgl. Burgard et al. 2019). Wie in Rupp (2018, Kap. 5) analysiert wurde, ist dieser Kalibrierungsschätzer von hoher numerischer Stabilität und somit auch auf große Datenmengen anwendbar. Durch dieses Verfahren würde eine kohärente Schätzung auf Gemeindeebene durch eine nachträgliche Kalibrierung erfolgen, die sowohl Register- als auch Stichprobeninformationen nutzt und dem Register dabei umso höheres Gewicht beimisst, je weniger Stichprobenanschriften in der betreffenden Gemeinde gezogen wurden. Auch eine Einbeziehung der Gemeindegröße ist dabei denkbar. Somit ist ein glatter Übergang gewährleistet, wobei sich die ausgewiesenen Schätzungen für sehr kleine Gemeinden (die Teil eines Verbandsgemeinderestes beziehungsweise einer Verbandsgemeinde sind) umso mehr an den vorhandenen Registerwerten orientieren wird, je geringer der Stichprobenumfang ist, der auf die entsprechende Gemeinde abfällt.

In Abb. 12 ist für die Alternativen A, B und C die Anzahl der Anschriften pro Gemeinde/SMP für jedes Bundesland separat als Boxplot abgebildet. Jeder Boxplot umfasst damit so viele Punkte wie Gemeinden/SMPs innerhalb des Bundeslandes vorhanden sind. Die Zahl hinter dem Boxplot zeigt diesen Wert noch einmal explizit an. Auffällig ist zum einen, dass sich die Größenstrukturen der Gemeinden/SMPs zwischen den Bundesländern teils signifikant unterscheiden. Beispielhaft haben die Bundesländer Schleswig-Holstein, Rheinland-Pfalz, Mecklenburg-Vorpommern und Thüringen sehr viele kleine Gemeinden (vergleiche Spalte zu Alternative A). Bei der Zusammenlegung dieser zu Verbandsgemeinden (Spalte zu Alternative C) ist somit in diesen Bundesländern ein besonders großer Effekt zu erwarten. Betrachtet man die Spalte zu Alternative C, so ist auffällig, dass Bayern, Sachsen und Thüringen im Verhältnis zu anderen Bundesländern kleine Verbandsgemeinden haben. Diese Tatsache wird sich in den resultierenden Stichprobenumfängen widerspiegeln. Zusätzlich ist zu erwähnen, dass sich die formale Definition von Verbandsgemeinden von Land zu Land unterscheiden kann, da es keine bundesweite einheitliche Definition gibt. 


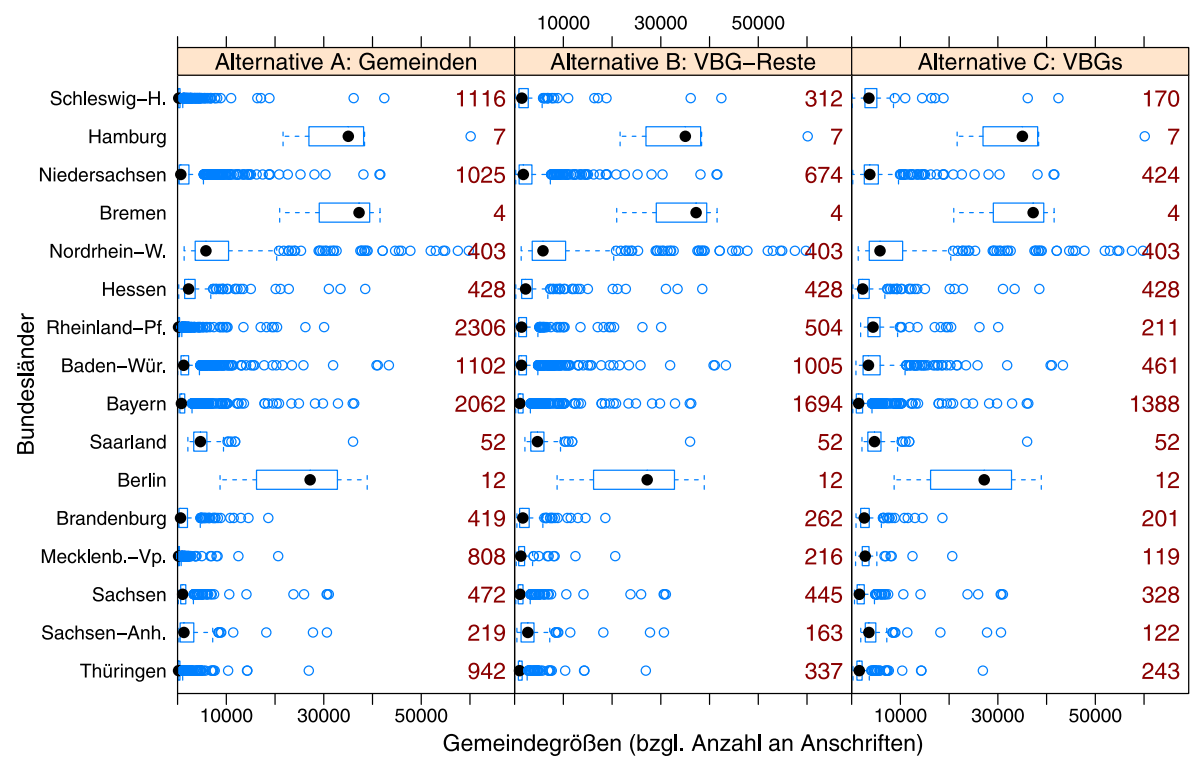

Abb. 12 Größe der Gemeinden, Gemeindeverbandsreste und Gemeindeverbände (Anzahl Anschriften) pro Land. Zahl zeigt Anzahl von Gemeinden / Gemeindeverbandsresten / Gemeindeverbänden im Boxplot.

Betrachtet man die Konfidenzintervallüberdeckungsraten für die drei neu definierten Ansätze in Abb. 13, so ergeben sich für alle drei Alternativen A, B und C sehr deutliche Verbesserungen im Vergleich zu der in Abb. 11 dargestellten Basisvariante. Die Konfidenzintervallüberdeckungsraten sind bei allen drei Alternativen für alle Gemeindegrößen akzeptabel, sodass aus dieser Sichtweise einer Anwendung von diesen Alternativen nichts im Wege steht. Zudem untermauern die deutlichen Verbesserungen im Vergleich zur Basisvariante in Abb. 11 die Verwendung einer der drei alternativen Ansätze zum Umgang mit Kleinstgemeinden.

Im Folgenden werden die Simulationsergebnisse für die zuvor erläuterten drei alternativen Schichtungsverfahren zum Umgang mit Kleinstgemeinden unter Verwendung von drei Präzisionszielfunktionen erläutert. Die zuvor untersuchte Auswahl der Box-Constraints für die Auswahlsätze wird im Folgenden auf 5\% und 50\% fixiert. Somit werden folgende Szenarien unterschieden:
A.1 Alternative A: GEM_100, Funktion: bez_1000_15, Box: 5\% und 50\%
A.2 Alternative A: GEM_100, Funktion: bez_1000_25, Box: 5\% und 50\%
A.3 Alternative A: GEM_100, Funktion: bez_1000_40, Box: 5\% und 50\%
B.1 Alternative B: VBG_Reste, Funktion: bez_1000_15, Box: 5\% und 50\%
B.2 Alternative B: VBG_Reste, Funktion: bez_1000_25, Box: 5\% und 50\%
B.3 Alternative B: VBG_Reste, Funktion: bez_1000_40, Box: 5\% und 50\%
C.1 Alternative C: VBG, Funktion: bez_1000_15, Box: 5\% und 50\%
C.2 Alternative C: VBG, Funktion: bez_1000_25, Box: 5\% und 50\%
C.3 Alternative C: VBG, Funktion: bez_1000_40, Box: 5\% und 50\% 

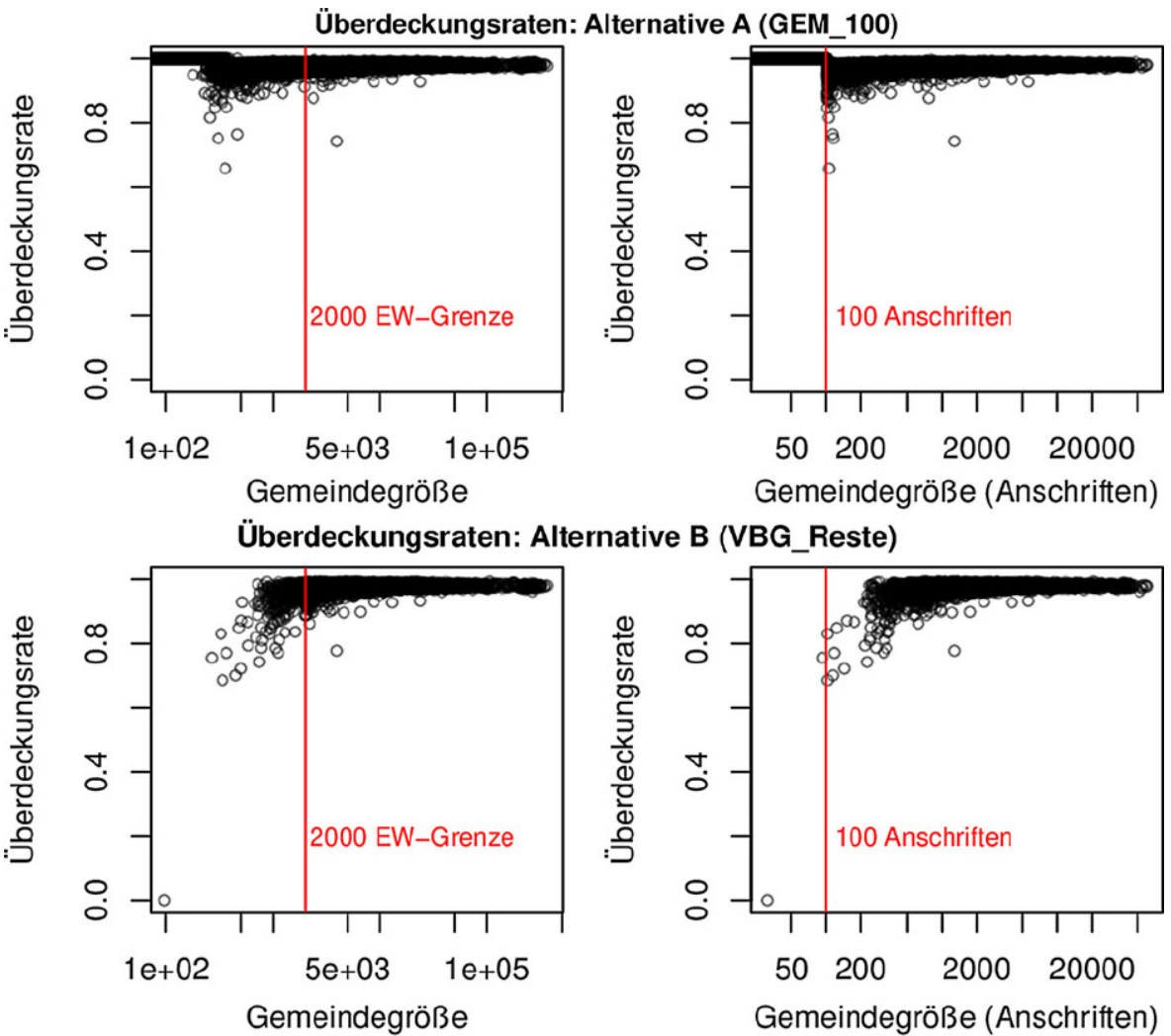

Überdeckungsraten: Alternative C (VBG)
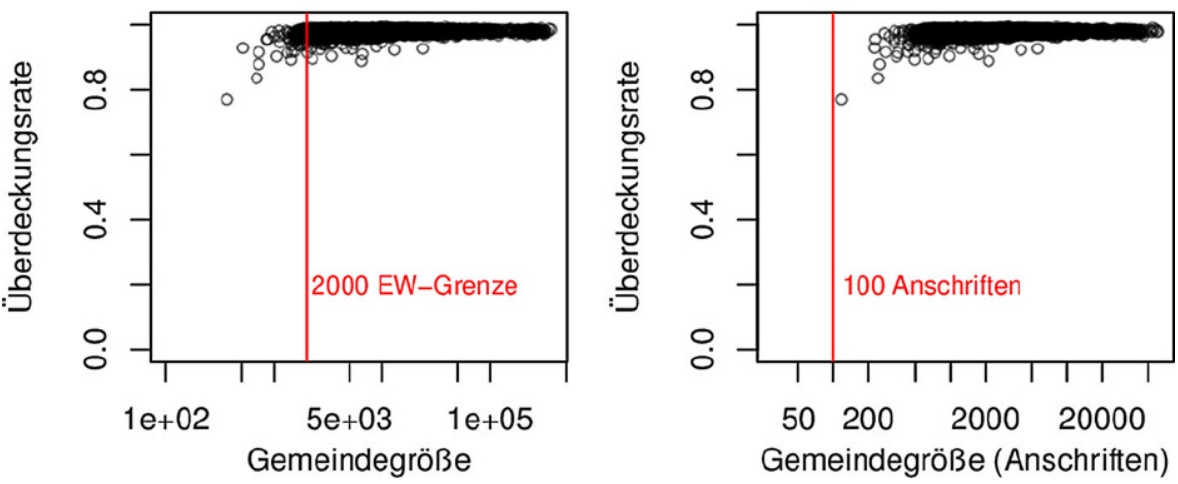

Abb. 13 Überdeckungsraten in Abhängigkeit der Gemeinde-, SMP- und VBG-Größe (Boxen:5\%, 50\%).

Die Ergebnisse der Simulation sind in Abb. 14 für Alternative A, in Abb. 15 für Alternative B und in Abb. 16 für Alternative C dargestellt (jeweils für bez_1000_15). Die Abbildungen sind analog zu Abb. 4 in Kap. 3.3 aufgebaut, wohingegen dort der Fall für eine fixe Definition von Kleinstgemeinden betrachtet wurde. Es ist hier zu erwähnen, dass sich die Auswertungen in Abb. 15 nur auf die 6.518 Sampling Points 
Relativer Standardfehler pro Gemeinde mit Funktion: bez_1000_15 (Gemeinde mit Boxen bei $5 \%$ und $50 \%$, mindestens aber 100 Anschriften)

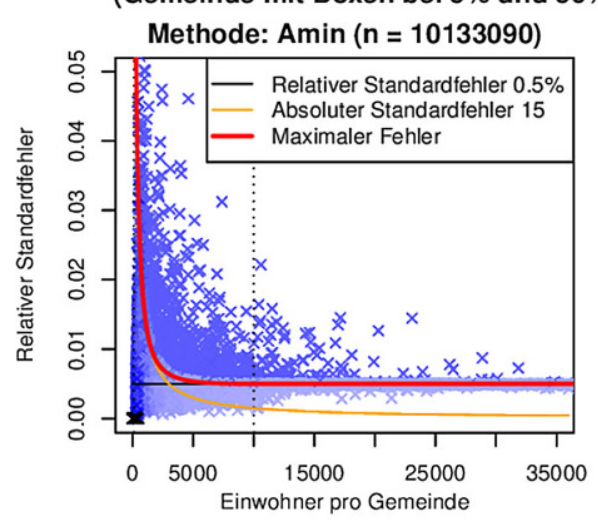

Relativer Standardfehler pro Gemeinde

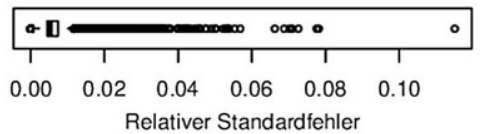

Fehlerhafte Gemeinden: 1100 von 11377

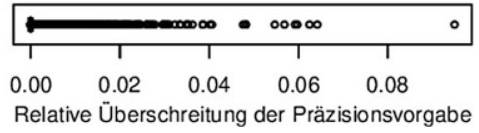

Methode: BCopt_0.00 ( $n=10130130)$

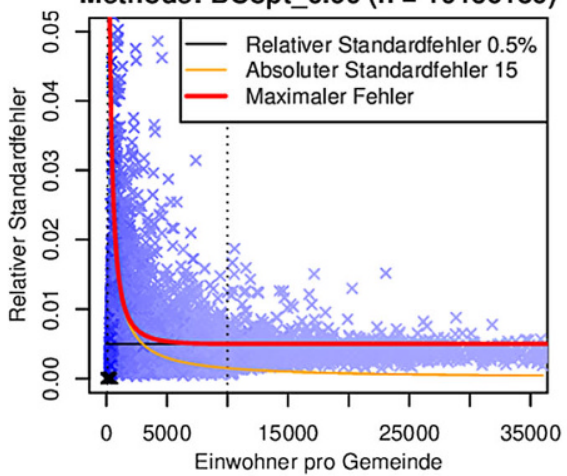

Relativer Standardfehler pro Gemeinde

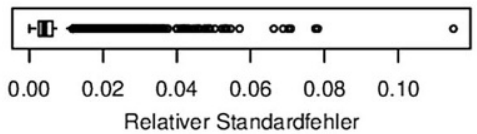

Fehlerhafte Gemeinden: 1377 von 11377

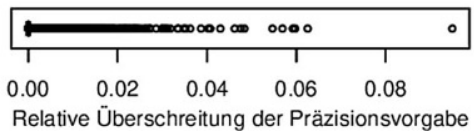

Abb. 14 Relativer Standardfehler bei Bézierkurve bez_1000_15 (für Alternative A: GEM_100)

(VBG_Reste) und die Auswertungen in Abb. 16 nur auf die 4.573 Verbandsgemeinden beziehen. Somit kann im Vergleich der Abbildungen kein direkter Rückschluss auf die Schätzqualität pro Gemeinde gezogen werden.

Im Vergleich zu Abb. 4 (Basisvariante) und Abb. 14 (Alternative A) sind in den Abb. 15 (Alternative B) und 16 (Alternative C) deutliche Unterschiede am linken Rand der oberen Plots zu erkennen. Die Punktewolke ist dort deutlich ausgedünnt, da es im Vergleich zu der hohen Anzahl kleiner Gemeinden nur noch sehr wenige kleine SMPs (VBG_Reste, VBGs) gibt. Somit vermeiden Alternativen B und C bis auf sehr wenige Ausnahmen eine Vollerhebung. Bei der Alternative A (Abb. 14) sind die Punkte im Bereich der kleinen Gemeinden teils deutlich dunkler als in Abb. 4, da es bei der Alternative A neben den Vollerhebungen in Gemeinden mit weniger als 100 Anschriften (ca. 1.000 Gemeinden) zahlreiche weitere kleine Gemeinden mit mehr als 100 Anschriften gibt, die auf Grund des Mindeststichprobenumfangs von 100 Anschriften einen erhöhten Auswahlsatz haben. Konträr zum Bereich der kleinen Gemeinden ist der Bereich ab 2.500 Einwohner in den Abb. 15 und 16 sehr ähnlich im Vergleich zu den Abb. 4 und 14. Der Hauptunterschied zwischen der Basisvariante und den Alternativen liegt somit im Bereich der Gemeinden unter 2.500 Einwohnern.

Der benötigte Gesamtstichprobenumfang für Alternative A liegt mit ca. 10,13 Millionen Personen um $15 \%$ über dem Stichprobenumfang für Alternative B (8,62 


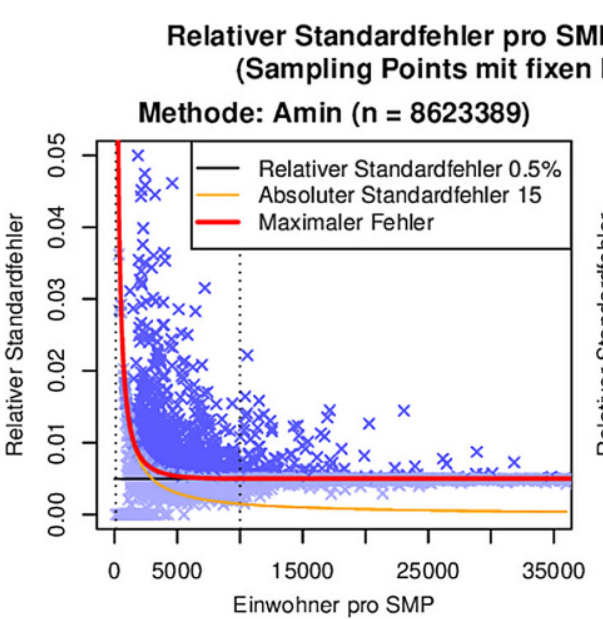

Relativer Standardfehler pro SMP

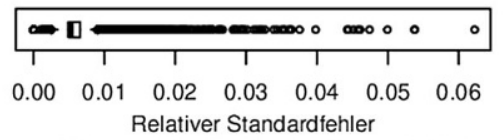

Fehlerhafte SMPs: 940 von 6518

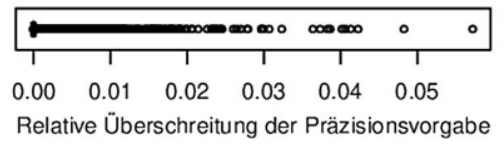

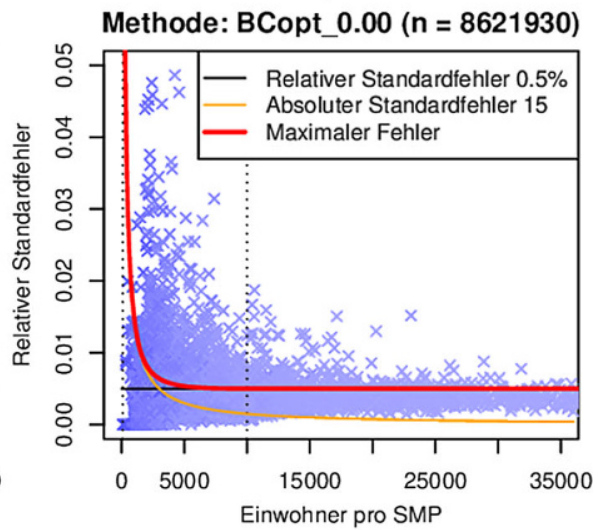

Relativer Standardfehler pro SMP

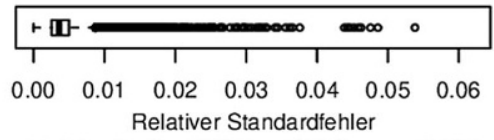

Fehlerhafte SMPs: 1238 von 6518

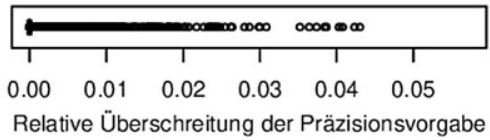

Abb. 15 Relativer Standardfehler bei Bézierkurve bez_1000_15 (für Alternative B: VBG_Reste)

Mio.). Dieser Anstieg beruht ausschließlich auf den deutlich höheren Auswahlsätzen für kleine Gemeinden, da ein Mindeststichprobenumfang von 100 Anschriften vorgeschrieben ist. Dies führt dann aber dazu, dass in kleinen Gemeinden einerseits Schätzwerte ausgewiesen werden, die auf der gleichen Methodik beruhen wie Schätzwerte für große Gemeinden. Darüber hinaus wird in Alternative A, im Vergleich zur Basisvariante (8,66 Mio.), die Schätzgüte in kleinen Gemeinden aufgrund der Grenze von mindestens 100 Anschriften erheblich verbessert. Dies wird insbesondere deutlich, wenn man die Boxplots der relativen Standardfehler pro Gemeinde zwischen Abb. 4 (Basisvariante) und Abb. 14 (Alternative A) vergleicht. Betrachtet man nun die Ergebnisse der Alternative $\mathrm{C}$ in Abb. 16, so sinkt der benötigte Gesamtstichprobenumfang im Vergleich zur Variante B um ca. 7,5\% (7,98 Mio.). Dies ist begründet in der geringeren Anzahl und gestiegenen Größe von Gemeindeverbänden im Vergleich zu Gemeindeverbandsresten. Somit ist festzuhalten, dass bei der Zusammenfassung von Gemeinden zu VBG-Resten beziehungsweise Verbandsgemeinden ein deutlicher Prozentsatz an Stichprobenumfang eingespart werden kann. Zudem werden auf Grund der geringen Anzahl kleiner Nachweiseinheiten Vollerhebungen fast vollständig vermieden. Bei den Alternativen B und C kann der Ausweis von gemeindespezifischen Schätzwerten durch synthetische Schätzungen beziehungsweise mit Hilfe des generalisierten Kalibrierungsschätzers (vgl. Burgard et al. 2019) erreicht werden. Die Anzahl der Gemeinden beziehungsweise SMPs, in 


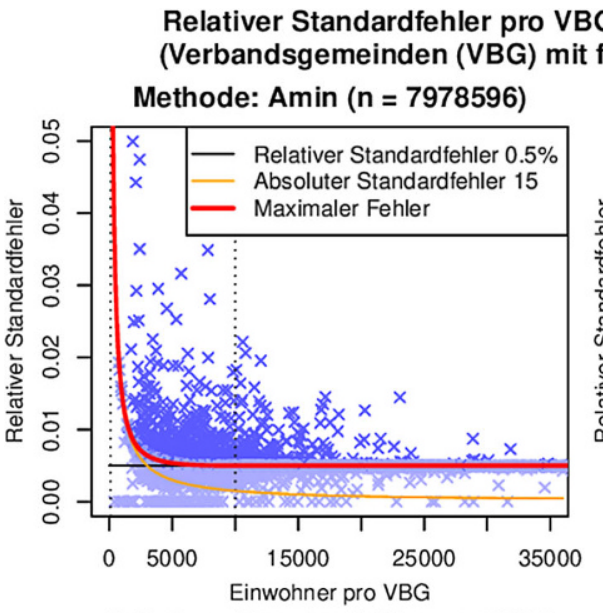

Relativer Standardfehler pro VBG

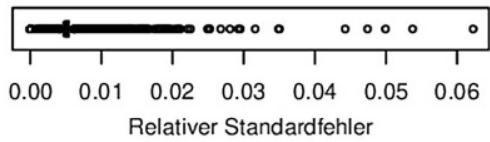

Fehlerhafte VBGs: $\mathbf{5 7 1}$ von $\mathbf{4 5 7 3}$

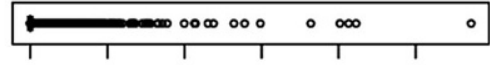

$\begin{array}{llllll}0.00 & 0.01 & 0.02 & 0.03 & 0.04 & 0.05\end{array}$

Relative Überschreitung der Präzisionsvorgabe



Relativer Standardfehler pro VBG

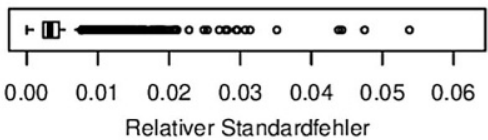

Fehlerhafte VBGs: 761 von 4573

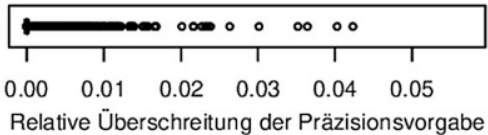

Abb. 16 Relativer Standardfehler bei Bézierkurve bez_1000_15 (für Alternative C: VBG)

denen die Präzisionsvorgabe nicht eingehalten wird, variiert dementsprechend auch zwischen den Varianten. Dies ist in den mittleren Zeilen der jeweiligen Abbildungen abzulesen.

In den folgenden Tabellen werden die benötigten Gesamtstichprobenumfänge für weitere Szenarien miteinander verglichen. Darüber hinaus sind diese exklusive eines etwaigen Sicherheitsaufschlagfaktors angegeben. Dieser wird erst in Kap. 4 näher untersucht. Die benötigten Gesamtstichprobenumfänge sind in Tab. 6 für die unterschiedlichen Szenarien in Abhängigkeit von Schichtung und Präzisionszielfunktion abgetragen. In den Zeilen sind die Präzisionszielfunktionen (d) bis (f) aus Kap. 3.2 abgebildet, die sich in der Wahl der absoluten Standardfehler zwischen 15, 25 und 40 unterscheiden. In den Spalten sind die Schichtungsmodelle aufgelistet. $\mathrm{Zu} \mathrm{Be}-$ ginn sind zwei Benchmarkszenarien mit einer gemeindescharfen Schichtung ohne Definition von Kleinstgemeinden (Gemeinden) und mit einer Definition von Kleinstgemeinden für Gemeinden kleiner als 100 Anschriften $(<100$ Anschr. Vollerhebung) abgebildet. Danach folgen die drei alternativen Ansätze mit gemeindescharfer Schichtung und Mindestauswahl von 100 Anschriften (Alternative A; GEM_100), der Schichtung mit Hilfe der Verbandsgemeinderesten (Alternative B; VBG_Reste) sowie der Schichtung mit Hilfe der Verbandsgemeinden (Alternative C; VBG). Im Vergleich zu einer konstanten Präzisionsvorgabe von einem relativen Standardfehler 
Tab. 6 Gesamtstichprobenumfang (Anzahl an Personen) im Vergleich zwischen gemeindespezifischer Schichtung beziehungsweise SMP-Schichtung

\begin{tabular}{lrrrrr}
\hline & Gemeinden & $\begin{array}{l}<100 \text { Anschr. } \\
\text { Vollerhebung }\end{array}$ & $\begin{array}{l}\text { Alternative A } \\
\text { (min. 100 } \\
\text { Anschr. }\end{array}$ & $\begin{array}{l}\text { Alternative B } \\
\text { (VBG-Reste) }\end{array}$ & $\begin{array}{l}\text { Alternative C } \\
(\text { VBG })\end{array}$ \\
\hline abs. \pm 15 & 8.662 .759 & 8.861 .209 & 10.130 .130 & 8.621 .930 & 7.978 .217 \\
abs. \pm 25 & 8.015 .813 & 8.223 .971 & 9.750 .724 & 8.140 .930 & 7.811 .922 \\
abs. \pm 40 & 7.366 .015 & 7.576 .630 & 9.256 .478 & 7.484 .732 & 7.491 .982 \\
\hline
\end{tabular}

von $0,5 \%$ für alle Gemeinden (diese Variante ist hier nicht aufgelistet) spart die Verwendung einer der Bézierfunktionen einen nicht unerheblichen Teil des Gesamtstichprobenumfangs ein. Zudem ist bei einer konstanten Präzisionsvorgabe trotz des hohen Gesamtstichprobenumfangs damit zu rechnen, dass sehr viele Gemeinden die Zielvorgabe nicht einhalten, da diese insbesondere für die kleinen Gemeinden zu restriktiv ist. Diese Methode ist also nicht praktikabel und ist dementsprechend auch nicht aufgelistet. Generell ist offensichtlich, dass der Stichprobenumfang ansteigt, wenn die Präzisionsvorgabe strikter wird (also im Verlauf von abs $\pm 40 \mathrm{zu}$ abs \pm 15$)$. Zudem benötigt eine Vollerhebung in Gemeinden unter 100 Anschriften im Vergleich zur Variante ohne explizite Sonderbetrachtung der kleinen Gemeinden einen Anstieg des Stichprobenumfangs von ca. 200.000 Einheiten. Der höchste Stichprobenumfang ist bei Alternative A zu erkennen, da die kleinen Gemeinden i. A. einen sehr hohen Auswahlsatz aufweisen. Wie zuvor schon beschrieben kann der Stichprobenumfang bei den Alternativen B und C deutlich geringer ausfallen.

In der Tab. 7 sind die bundeslandspezifischen Stichprobenumfänge (Anzahl an Personen) für die neun zu Beginn des Abschnittes genannten Szenarien dargestellt. Diese entsprechen den Szenarien in Spalte vier bis sechs der Tab. 6. Die entsprechenden Auswahlsätze sind in der Tab. 8 dargestellt, wodurch sich die Bundesländer besser miteinander vergleichen lassen. Sehr auffällig sind die sehr hohen Auswahlsätze in den Bundesländern Schleswig-Holstein, Rheinland-Pfalz, Mecklenburg-Vorpommern und Thüringen im Fall der Verwendung der Alternative A. Diese können im Fall der Verwendung der Gemeindeverbandsreste (Alternative B) und Gemeindeverbände (Alternative $C$ ) jedoch deutlich reduziert werden, sodass die Auswahlsätze dieser Bundesländer insbesondere bei der Alternative C im Durchschnitt aller Länder liegen. Die vier genannten Bundesländer sind die, in denen sich sehr viele kleine Gemeinden befinden (vgl. Abb. 12). Als Folge dessen werden dort in den Alternativen $\mathrm{B}$ und $\mathrm{C}$ viele dieser kleinen Gemeinden zusammengelegt. Generell hängt die Höhe der Reduktion des Stichprobenumfangs von Alternative A auf B oder C von der Struktur der Gemeinden und Verbandsgemeinden in den Bundesländern ab. Betrachtet man die Ergebnisse anderer Länder wie Nordrhein-Westfalen oder das Saarland, so ergeben sich meist nur marginale Veränderungen zwischen den Alternativen $\mathrm{A}, \mathrm{B}$ und $\mathrm{C}$, die hauptsächlich durch die kompensatorische Allokation via BCopt zustande kommen. Letztendlich sind Auswahlsätze bei Alternative B und im Speziellen bei Alternative $C$ ausgeglichener über die Bundesländer verteilt.

Die Anzahl der Gemeinden, VBG-Reste beziehungsweise VBGs, die einen erhöhten Auswahlsatz (also über der eigentlichen Box von 50\%) aufweisen, sind in Tab. 9 für die Alternativen A, B und C aufgelistet (beispielhaft mit Bézierkurve 


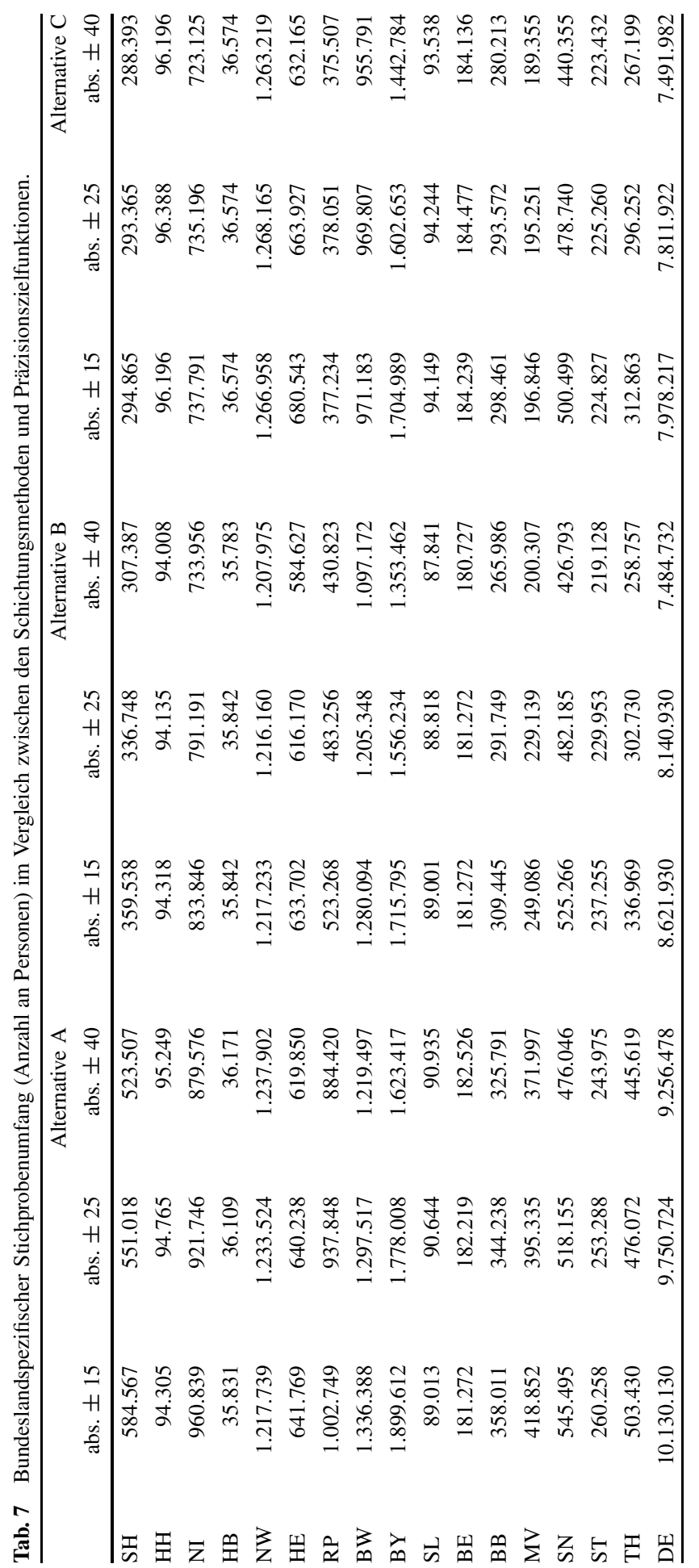




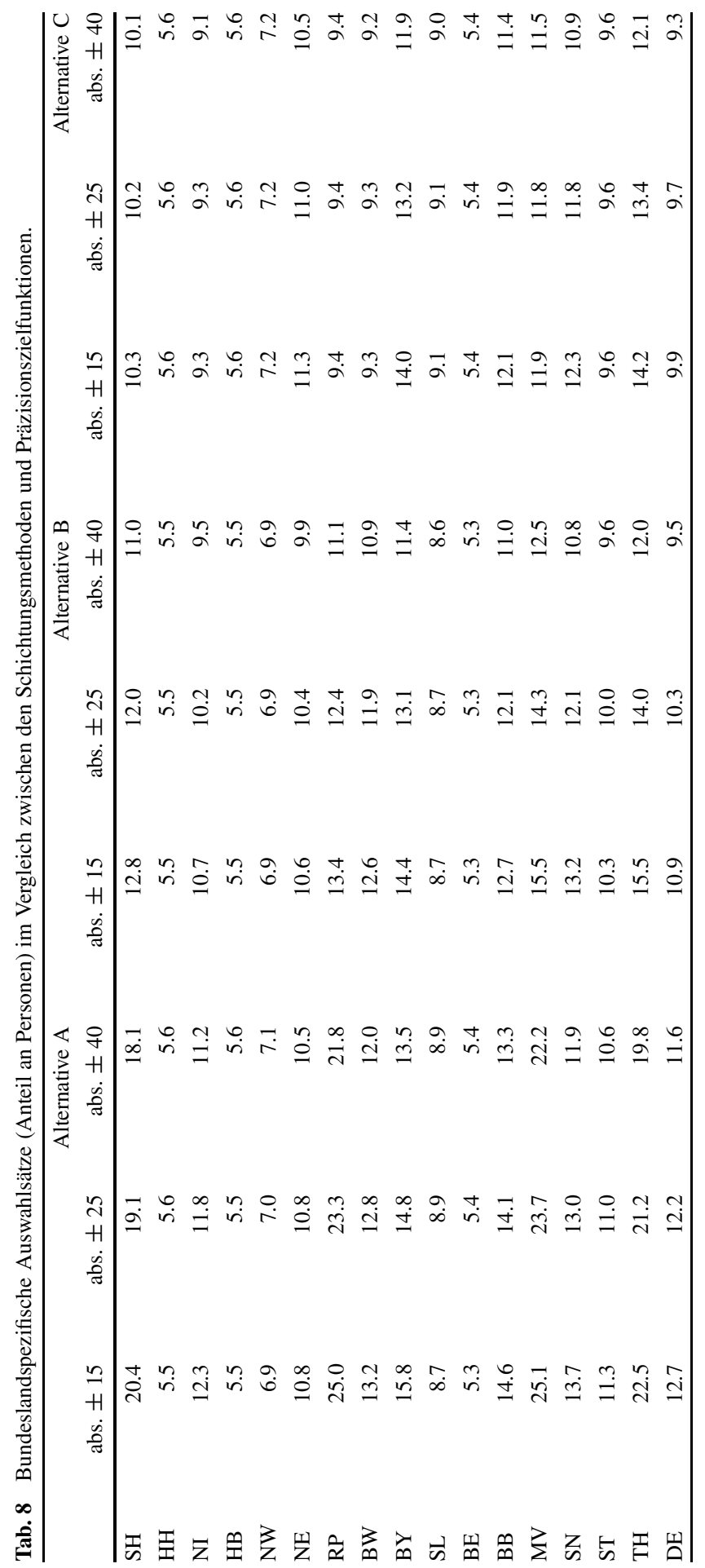




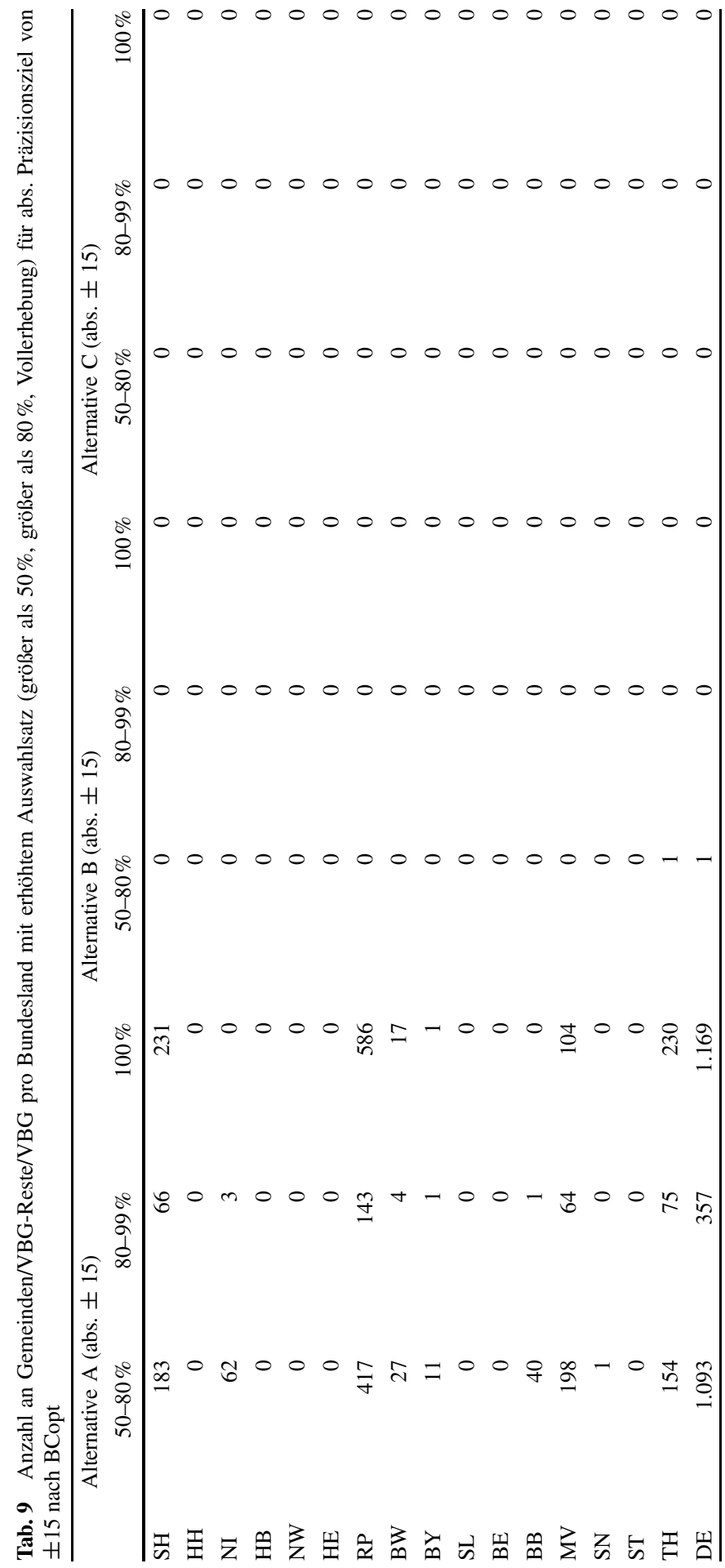


mit abs. Standardfehler von 15). Dabei wird zwischen Auswahlsätzen von $50 \%$ und $80 \%$, $80 \%$ und $<100 \%$, sowie Vollerhebung unterschieden. Offensichtlich gibt es bei der Alternative A eine hohe Anzahl von Vollerhebungsschichten, insgesamt wird also in 1.169 von 11.377 (also in über 10\%) der Gemeinden eine Vollerhebung durchgeführt. Darüber hinaus ergibt sich bei Alternative A noch eine nicht unerhebliche Zahl an Gemeinden mit erhöhten Auswahlsätzen. Dies resultiert aus der Mindeststichprobengröße von 100 Anschriften pro Gemeinde. Darüber hinaus ist auffällig, dass die Gemeinden mit erhöhten Auswahlsätzen nicht gleichverteilt über alle Bundesländern sind, sondern in erhöhter Form in den oben schon genannten vier Bundesländern Schleswig-Holstein, Rheinland-Pfalz, MecklenburgVorpommern und Thüringen vorkommen.

Als Zusammenfassung der Alternativen A, B und C lässt sich also festhalten, dass alle drei Alternativszenarien Vor- und Nachteile haben. Die Alternativen B und C mit einer Schichtung nach Verbandsgemeinderesten beziehungsweise Verbandsgemeinden benötigen einen deutlich geringeren Gesamtstichprobenumfang und kommen ohne Vollerhebungsschichten aus, haben aber den Nachteil, dass keine direkten Schätzungen für die einzelnen Gemeinden innerhalb eines SMP möglich sind. Eine Ausweisung der Schätzung ist nur bei Alternative A durch die gemeindescharfe Schichtung für alle Gemeinden möglich, allerdings sind in ca. 10,3\% der Gemeinden dafür Vollerhebungen von Nöten. Zudem haben weitere 1.450 (ca. 12,7\%) Gemeinden einen über der Grenze von 50\% liegenden Auswahlsatz. Aufgrund dessen ist der Gesamtstichprobenumfang somit bei Alternative A auch deutlich höher. Ein weiterer entscheidender Unterschied sind die bundeslandspezifischen Auswahlsätze, die in Tab. 8 abgebildet sind. Die Bundesländer mit vielen kleinen Gemeinden weisen in Alternative A einen sehr hohen Auswahlsatz aus. Dieser wird durch die Zusammenfassung kleiner Gemeinden in Alternative B und C wieder angeglichen. Die Angleichung dieser ungleichen Auswahlsätze sowie der Verzicht von Vollerhebungsschichten sind insbesondere innerhalb der Länder mit problematischen Strukturen deutliche Argumente für die Verwendung der Schichtung anhand der Verbandsgemeinderesten (Alternative B) beziehungsweise Verbandsgemeinden (Alternative C). Im Allgemeinen ist auch eine länderspezifische Wahl der Methodik möglich.

\section{Bestimmung des Gesamtstichprobenumfangs}

Die Berechnung des Gesamtstichprobenumfangs in Kap. 3 basiert auf den geschätzten Varianzreduktionsfaktoren $\left(1-\vartheta^{2}\right)$ durch die Verwendung des GREG-Ansatzes (siehe Kap. 3.4). Diese gemeindespezifischen Varianzreduktionsfaktoren sind somit keine feste Größen, sondern Ausprägungen von Zufallsvariablen. Um sicher zu stellen, dass die Präzisionsvorgaben eingehalten werden, ist es erforderlich, einen Sicherheitsaufschlagfaktor zu verwenden, der die Unsicherheit der Schätzung der Varianzreduktionsfaktoren berücksichtigt. Dazu werden im Folgenden zwei Varianten vorgestellt: 
4.1 Erhöhung des Varianzreduktionsfaktors $\left(1-\vartheta^{2}\right)$ für jede(n) Gemeinde/SMP um eine geschätzte Standardabweichung der Schätzung $\left(1-\widehat{\vartheta}^{2}\right)$. Berechnung der geschätzte Standardabweichung auf Basis eines Bootstraps.

4.2 Erhöhung des Varianzreduktionsfaktors $\left(1-\vartheta^{2}\right)$ für jede(n) Gemeinde/SMP um den Wert, der nötig ist, um die $95 \%$ ige Überdeckung des $95 \%$-Konfidenzintervalls für die Schätzung der Einwohnerzahl für jede(n) Gemeinde/SMP zu gewährleisten.

Beide Varianten werden im Folgenden in je einem Unterkapitel erläutert. Die Bootstrap-Variante 4.1 wird beispielhaft für das folgende Szenario vorgestellt: Schichtung via Gemeinden mit einer Vollerhebung in Gemeinden kleiner als 100 Anschriften, Boxen 5\% - 50\% und Zielfunktion bez_1000_25. Die zweite Variante mit den Konfidenzintervallüberdeckungsraten wird für alle Basisvarianten und Alternativen sowie drei Präzisionszielfunktionen durchgerechnet, um so einen guten Vergleich der Aufschlagfaktoren in Abhängigkeit von Schichtungsvariante und Zielfunktion zu erhalten.

\subsection{Sensitivität des Reduktionsfaktors via MC-Bootstrap}

Da keine neueren Informationen zur Qualität der Register und der Erklärungskraft des assistierenden Modells des GREG vorliegen, müssen die Varianzreduktionsfaktoren auf Basis des Zensus 2011 geschätzt werden. Die Unsicherheit der Schätzung kann anhand des klassischen Monte-Carlo Bootstraps (siehe Särndal et al. 1992, Kap. 11.6) berechnet werden. Hierbei wird wiederholt in jeder Schicht durch Ziehen mit Zurücklegen die gleiche Anzahl an Elementen gezogen wie sie auch in der ursprünglichen Stichprobe gezogen wurden. Auf diesem Resample wird der Varianzreduktionsfaktor erneut berechnet. Die Verteilung der auf den Resamples berechneten Varianzreduktionsfaktoren ist eine Schätzung für die tatsächliche Verteilung der Varianzreduktionsfaktoren. Da der Varianzreduktionsfaktor eine nichtlineare Statistik ist, ist es nicht offensichtlich, welcher Sicherheitsaufschlagfaktor im Erwartungswert ausreichend sein sollte. Deswegen wird anhand einer Monte-Carlo Simulation auf der synthetischen Simulationsgesamtheit überprüft, was ein plausibler Sicherheitsaufschlagfaktor sein könnte. Die Simulation verläuft in folgenden Schritten:

1. Ziehe eine Stichprobe aus dem synthetischen Datensatz auf die Art, wie sie im Zensus 2011 gezogen wurde.

2. Schätze den in dieser Stichprobe resultierenden Varianzreduktionsfaktor für jede Gemeinde.

3. Führe einen Monte-Carlo Bootstrap durch und berechne in jeder Bootstrap-Iteration den Varianzreduktionsfaktor für jede Gemeinde.

4. Überprüfe, ob der um den Sicherheitsaufschlagfaktor korrigierte Varianzreduktionsfaktor eine konservative Abschätzung des tatsächlichen Varianzreduktionsfaktors darstellt.

5. Wiederhole Schritte 1.-4. insgesamt 999 Mal. 
Abb. 17 Boxplots für vier Gemeinden von aus Stichprobe geschätztem Reduktionsfaktor mit und ohne Aufschlagfaktor (eine Standardabweichung der Reduktionsfaktoren aus den Bootstrap-Stichproben).

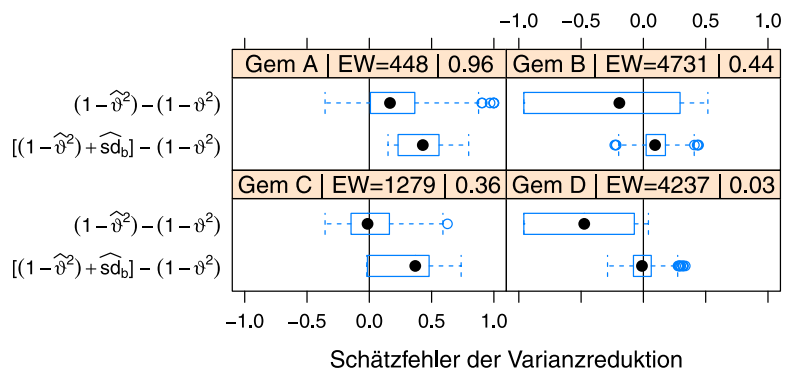

Vor der Auswertung der Simulationsergebnisse ist nun zu diskutieren, wie der Sicherheitsaufschlagfaktor zu wählen ist. Generell wird bei einem Bootstrap angenommen, dass die Verteilung der Varianzreduktionsfaktoren durch die BootstrapStichproben eine Schätzung für die tatsächliche Verteilung der Varianzreduktionsfaktoren ist. Somit ließe sich die Variabilität der durch die Stichproben geschätzten Varianzreduktionsfaktoren bezüglich der theoretische korrekten, aber in der Realität unbekannten, Varianzreduktionsfaktoren als Variabilität der durch die BootstrapReplikationen geschätzten Varianzreduktionsfaktoren abbilden. Die geschätzte Standardabweichung $\widehat{\operatorname{sd}}_{\text {Boot }}(\widehat{\vartheta})$ der Bootstrap-Schätzungen wird als sinnvoller Kandidat für die Wahl eines Aufschlagfaktors angesehen. Diese theoretisch fundierte Wahl eines Aufschlagfaktors ist der naiven Wahl eines konstanten Aufschlagfaktors vorzuziehen.

In Abb. 17 sind die Auswirkungen der Aufschlagfaktorberechnung mittels der Standardabweichung der Bootstrap-Schätzungen beispielhaft für vier zufällig ausgewählte Gemeinden dargestellt. Die Boxplots in der jeweils ersten Zeile beinhalten 1.000 Punkte (für jede MC-Wiederholung) und zeigen den Schätzfehler der Varianzreduktion ohne Aufschlagfaktor, also die Differenz aus tatsächlichem Aufschlagfaktor $\left(1-\vartheta^{2}\right)$ und geschätztem Aufschlagfaktor $\left(1-\widehat{\vartheta}^{2}\right)$. Für alle Stichproben mit Punkten rechts von der Null-Linie ist also die Eigenschaft einer konservativen Schätzung erfüllt. In der jeweils zweiten Zeile sind die Schätzfehler der Varianzreduktion mit Aufschlagfaktor abgetragen. Dabei entspricht $\widehat{s d}_{b}$ der Standardabweichung der Bootstrap-Schätzungen für den Varianzreduktionsfaktor. Es ist deutlich zu erkennen, dass die Wahrscheinlichkeit einer konservativen Schätzung unter Verwendung des Aufschlagfaktors deutlich ansteigt. Zur weiteren Information sind im Titel des jeweiligen Panels sowohl die Einwohnerzahl als auch der tatsächliche Varianzreduktionsfaktor zu sehen. Alles in allem ist an der Breite der Boxplots aber die hohe Instabilität der Schätzung des Varianzreduktionsfaktors erkennbar, sodass eine so deutliche Korrektur wie $+\widehat{s d}_{\mathrm{b}}$ nicht $\mathrm{zu}$ umgehen ist. Ein entscheidender Faktor zur Instabilität ist die oft sehr heterogene obere Schicht der Gemeinde (große Anschriften). Um dies genauer zu erläutern, sind im Anhang (Abb. 22 bis 26) weitere Detailauswertungen zu den vier ausgewählten Gemeinden und Stichproben zu finden.

In der folgenden Abb. 18 ist prinzipiell der gleiche Sachverhalt wie in Abb. 17 dargestellt, jedoch über alle Gemeinden. Um eine Darstellung zu ermöglichen, ist nur der Mittelwert (erste Spalte) beziehungsweise der Median (zweite Spalte) der jeweiligen 1.000 MC-Durchläufe pro Gemeinde abgetragen. Somit beinhaltet jeder 
Abb. 18 Für alle Gemeinden: Boxplots von Median und Mittelwert der aus Stichprobe geschätztem Reduktionsfaktor mit und ohne Aufschlagfaktor.

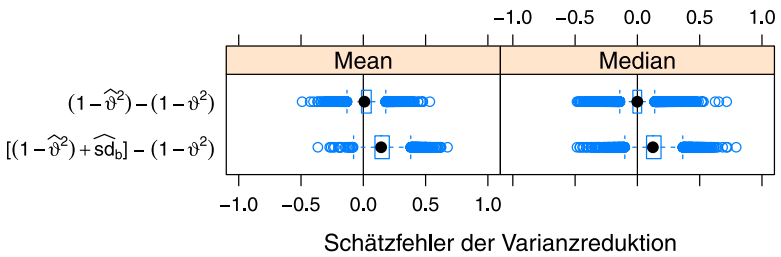

Boxplot 11.377 Punkte. Auch in dieser Abbildung ist ein ähnliches Verhalten zu erkennen wie in Abb. 17. Im Fall mit Aufschlagfaktor ist der Boxplot deutlich nach rechts verschoben. In beiden Fällen (Mittelwert und Median) sind nur noch Aufschlagfaktoren für wenige Gemeinden als nicht konservativ zu bezeichnen. Die Aufschlagfaktoren tragen somit sehr deutlich zur Erhöhung der Wahrscheinlichkeit einer konservativen Schätzung der Varianzreduktionsfaktoren bei.

Um das Verfahren weiter zu analysieren, betrachten wir nun die Abb. 19 und 20. Der linke Graph bezieht sich jeweils auf das Szenario ohne Aufschlagfaktor, der rechte auf das mit integriertem Aufschlagfaktor. Auf der Abszisse ist jeweils der Anteil an den Gemeinden abgetragen. Auf der Ordinate ist der Anteil der konservativen Schätzungen pro Gemeinde, berechnet durch die MC-Simulation, abgetragen. Das bedeutet, dass für eine Gemeinde mit einem Wert von 0,50 genau 500 von 1.000 der geschätzten Varianzreduktionsfaktoren $\left(1-\widehat{\vartheta}^{2}\right)$ der Gemeinde den tatsächlichen Varianzreduktionsfaktor überschreitet, also eine konservative Schätzung für den Varianzreduktionsfaktor ist. Die gestrichelte Linie zeigt die Grenze, ab der $50 \%$ aller MC-Durchläufe diese Eigenschaft erfüllen; die Überschreitung dieser Linie wird als besonderes Gütekriterium angesehen. Beim rechten Plot sind die Aufschlagfaktoren hinzugerechnet. In Abb. 19 ist dies in Abhängigkeit der Gemeindegröße dargestellt (unterteilt in drei Größenklassen). Abbildung 20 zeigt dies in Abhängigkeit des tatsächlichen Varianzreduktionsfaktors (unterteilt in drei Klassen). Im Hinblick auf die Gemeindegröße (Abb. 19) ist erkennbar, dass bei einer Verwendung der Varianzreduktionsfaktoren diese im Mittel nur in der Hälfte aller Gemeinden ei-
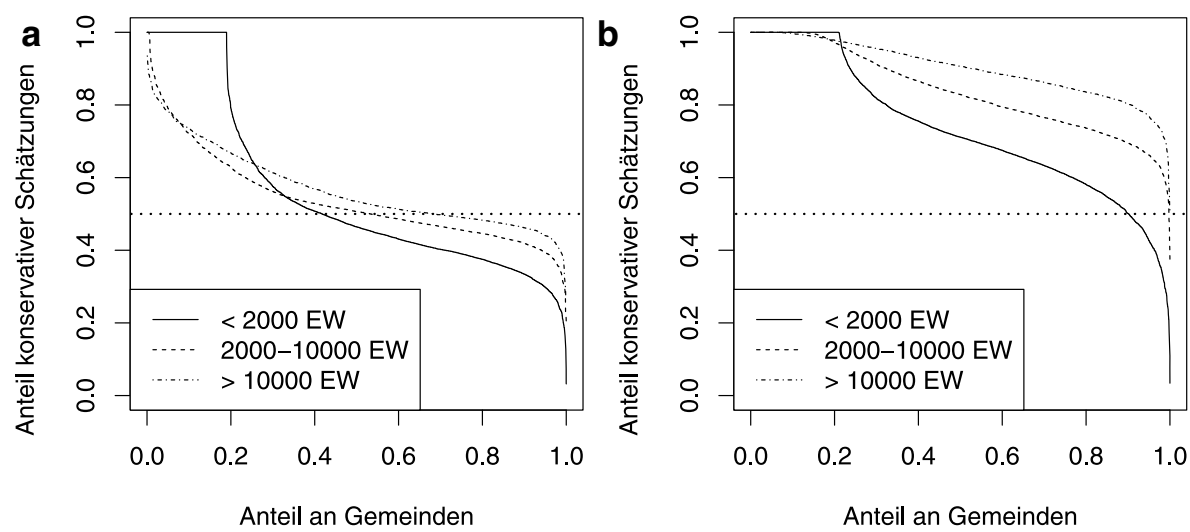

Abb. 19 Anteil an den 1.000 MC-Durchläufen, in denen der Mittelwert der Reduktionsfaktoren aus den Bootstrap-Stichproben mit und ohne Aufschlagfaktor den aus der Stichprobe geschätzten Faktor übersteigt 



Abb. 20 Anteil an den 1.000 MC-Durchläufen, in denen der Mittelwert der Reduktionsfaktoren aus den Bootstrap-Stichproben mit und ohne Aufschlagfaktor den aus der Stichprobe geschätzten Faktor übersteigt

ne konservative Schätzung der tatsächlichen Varianzreduktionsfaktoren darstellen. Dabei ist ein ähnlicher Verlauf für alle drei Größenklassen von Gemeinden zu erkennen. Wird nun der Aufschlagfaktor mitberücksichtigt (rechter Plot), so ergibt sich eine deutlich veränderte Struktur. Mehr als 90\% der Varianzreduktionsfaktoren plus Aufschlagfaktor für kleine Gemeinden und nahezu 100\% der Faktoren für mittlere und große Gemeinden erfüllen im Mittel die Eigenschaft einer konservativen Schätzung in Bezug auf die tatsächlichen Varianzreduktionsfaktoren. Betrachtet man nun den Anteil konservativer Schätzungen in Bezug auf die Höhe der tatsächlichen Varianzreduktionsfaktoren (siehe Abb. 20), so ergibt sich ein ähnliches Bild. Erneut führt die Inklusion der Aufschlagfaktoren zu einer deutlichen Steigerung des Anteils einer konservativen Schätzung. Darüber hinaus ist an der linken und rechten Grafik zu erkennen, dass Gemeinden mit kleinen Varianzreduktionsfaktoren (also hoher Varianzreduktion zwischen HT- und GREG-Schätzer) einen höheren Anteil an konservativer Schätzungen aufweisen.

Zur Einschätzung des Befragungsmehraufwands ist die Analyse des resultierenden Gesamtstichprobenumfangs von entscheidender Bedeutung. Die Folgen der Verwendung des Aufschlagfaktors sind in diesem Fall enorm. Der Gesamtstichprobenumfang würde um ca. $42 \%$ ansteigen. Besonders auffällig ist die Konstanz der Aufschlagfaktoren über alle MC-Durchläufe. Da der numerische Aufwand für die Durchführung eines solchen Bootstraps zur Bestimmung des Sicherheitsaufschlagfaktor durch die Erhöhung des Varianzreduktionsfaktors um eine Standardabweichung der Bootstrap-Schätzverteilung sehr hoch ist, wird im Folgenden eine weitere alternative Methode vorgestellt, um die Werte zu verifizieren und eine kostengünstigere Möglichkeit aufzuzeigen.

\subsection{Konfidenzintervallüberdeckungsraten für Schätzungen}

In diesem Unterkapitel wird eine alternative Variante zur Berechnung des Sicherheitsaufschlagfaktors für die Varianzreduktionsfaktoren vorgestellt. Dazu werden die 
Tab. 10 Benötigte Aufschlagfaktoren pro Szenario und Präzisionszielfunktion.

\begin{tabular}{lrrrrr}
\hline & Gemeinden & $\begin{array}{l}<100 \text { Anschr. } \\
\text { Vollerhebung }\end{array}$ & $\begin{array}{l}\text { Alternative A } \\
\text { (min. 100 } \\
\text { Anschr.) }\end{array}$ & $\begin{array}{l}\text { Alternative B } \\
\text { (VBG-Reste) }\end{array}$ & \multicolumn{1}{l}{$\begin{array}{l}\text { Alternative C } \\
\text { (VBG) }\end{array}$} \\
\hline abs. \pm 15 & $31,00 \%$ & $27,06 \%$ & $5,94 \%$ & $6,85 \%$ & $5,02 \%$ \\
abs. \pm 25 & $43,24 \%$ & $37,95 \%$ & $5,92 \%$ & $7,07 \%$ & $4,90 \%$ \\
abs. \pm 40 & $51,10 \%$ & $44,93 \%$ & $5,84 \%$ & $7,27 \%$ & $5,21 \%$ \\
\hline
\end{tabular}

Konfidenzintervallüberdeckungsraten des $95 \%$ Konfidenzintervalls verwendet. Die Vorgehensweise ist anhand der folgenden Schritte zu erklären:

1. Basis ist der durch Amin berechnete Stichprobenumfang.

2. Berechnung der Konfidenzintervallüberdeckungsraten pro Gemeinde anhand einer MC-Simulation mit 1.000 Wiederholungen.

3. Erhöhung des Stichprobenumfangs bei den Gemeinden/SMPs, bei denen die Konfidenzintervallüberdeckungsrate unter $95 \%$ liegt. Die sukzessive Erhöhung wird gestoppt, sobald eine Konfidenzintervallüberdeckungsraten von $95 \%$ erreicht ist.

4. Aus dem in diesem Fall zusätzlich benötigten Stichprobenumfang wird der benötigte Sicherheitsaufschlagfaktor berechnet.

In Kap. 3.6 wurde anhand von Abb. 11 bereits erklärt, dass bei einer gemeindespezifischen Schichtung und Vollerhebung von Gemeinden kleiner als 100 Anschriften insbesondere bei Gemeinden kleiner als 2.000 Einwohnern (vertikale Linie im rechten Plot) die Konfidenzintervallüberdeckungsraten problematisch sind. Daher ist auch ein deutlicher Aufschlagfaktor für den Gesamtstichprobenumfang vonnöten, um dies zu korrigieren. Betrachtet man die zuvor schon erwähnten Alternativen A, B und C in Abb. 13, so ergibt sich ein deutlich verändertes Bild. Die Konfidenzintervallüberdeckungsraten sind im Schnitt deutlich höher, sodass nur ein signifikant kleinerer Aufschlagfaktor notwendig ist, um einer konservative Schätzung des Gesamtstichprobenumfangs gerecht zu werden.

In Tab. 10 sind die benötigten prozentualen Sicherheitsaufschlagfaktoren dargestellt. Dabei wird zwischen den bereits in Kap. 3.6 genannten Basisvarianten mit gemeindescharfer Schichtung ohne Definition von Kleinstgemeinden (Gemeinden) und mit einer Definition von Kleinstgemeinden für Gemeinden mit weniger als 100 Anschriften ( $<100$ Anschr. Vollerhebung) sowie zwischen den Alternativen A, B und $\mathrm{C}$ unterschieden (jeweils für die drei betrachteten Bézierkurven). Es ist ein sehr deutlicher Unterschied zwischen den beiden Basisvarianten und den drei alternativen Schichtungsvarianten zu erkennen. Wie zudem aus Abb. 13 abzuleiten ist, kommen die Alternativen A, B und C mit deutlich geringeren Aufschlagfaktoren aus. Diese liegen durchweg im Bereich zwischen $5 \%$ und 7,5\%. Vergleicht man das Ergebnis mit der Methode in Kap. 4.1 (Szenario: < 100 Anschriften, bez_1000_25), in welcher der Varianzreduktionsfaktor um eine Standardabweichung erhöht wurde, so ergibt sich ein sehr ähnliches Ergebnis. Während nach der Methode aus Kap. 4.1 eine Erhöhung des Stichprobenumfangs um $42 \%$ benötigt wird, ergibt sich hier eine Erhöhung von $38 \%$. Somit ist zumindest anhand dieses Beispiels gezeigt, dass beide Methoden ähnliche Ergebnisse liefern. 


\subsection{Festlegung des Gesamtstichprobenumfangs für den Zensus 2021}

Um abschließend eine konservative Schätzung für den benötigten Gesamtstichprobenumfang für den Zensus 2021 abgeben zu können, werden die Ergebnisse aus den Kap. 3.6, 4.1 und 4.2 verknüpft. Dazu werden den berechneten Stichprobenumfängen die ermittelten Aufschlagfaktoren hinzugerechnet, um eine konservative Schätzung zu erhalten und stabile Schätzungen im Zensus 2021 zu ermöglichen. Die Gesamtstichprobenumfänge sind in Tab. 11 aufgelistet. Dabei dienen die Szenarien „Gemeinden“ und „,< 100 Anschr. Vollerhebung“ in den Spalten 2 und 3 als Benchmarkszenarien. In den folgenden Spalten sind die benötigten Stichprobenumfänge inklusive Aufschlagfaktoren für die in Kap. 3.6 vorgestellten Alternativen A, B und $\mathrm{C}$ abgetragen. Somit ergibt sich eine Spanne des Stichprobenumfangs von 7,88 Mio. bis 11,48 Mio. für die jeweiligen Szenarien.

Zur Einschätzung der Belastung der einzelnen Bundesländer sind die bundeslandspezifischen Stichprobenumfänge in Tab. 12 und die bundeslandspezifischen Auswahlsätze in Tab. 13 dargestellt. Auffällig ist die signifikante Reduktion des benötigten Gesamtstichprobenumfangs in den Bundesländern Schleswig-Holstein, Rheinland-Pfalz, Mecklenburg-Vorpommern und Thüringen im Fall der Verwendung der Gemeindeverbandsreste (Alternative B) und Gemeindeverbände (Alternative C). Die übrigen geringfügigen Unterscheidungen kommen hauptsächlich durch die kompensatorische Allokation via BCopt zustande. Letztendlich ist festzuhalten, dass die Auswahlsätze bei Alternative $B$ und im Speziellen bei Alternative $C$ ausgeglichener über die Bundesländer verteilt sind. Die beschriebene Angleichung der bundeslandspezifischen Auswahlsätze bei den Alternativen B und C sowie der vollständige Verzicht auf Vollerhebungsschichten führt insbesondere in den Ländern mit vielen kleinen Gemeinden (z. B. Schleswig-Holstein, Rheinland-Pfalz, Mecklenburg-Vorpommern und Thüringen) zu einer Reduktion des benötigten Stichprobenumfangs im Vergleich zu Alternative A.

Zur erweiterten Analyse der Ergebnisse sind in Tab. 14 die Auswahlsätze für die Alternativen A, B und C in Abhängigkeit der SMPs-Größe (unterteilt in Anzahl der Anschriften pro SMP) aufgelistet. Die Auswahlsätze in kleinen Gemeinden, VBGResten beziehungsweise VBGs liegen in allen drei Alternativen deutlich höher als die Auswahlsätze in großen Gemeinden, VBG-Resten beziehungsweise VBGs. Des Weiteren ist auffällig, dass auf kleine Gemeinden bei Alternative A eine enorme Belastung zukommt, während die Belastung für diese Gemeinden bei den Alternativen B und C abnimmt. Generell sind die Auswahlsätze bei Alternative B, aber insbe-

Tab. 11 Konservative Schätzung des Gesamtstichprobenumfangs (Anzahl an zu befragenden Personen) inklusive Aufschlagfaktor.

\begin{tabular}{lcrrrr}
\hline & Gemeinden & $\begin{array}{l}<100 \text { Anschr. } \\
\text { Vollerhebung }\end{array}$ & $\begin{array}{l}\text { Alternative A } \\
\text { (min. 100 } \\
\text { Anschr. }\end{array}$ & $\begin{array}{l}\text { Alternative B } \\
\text { (VBG-Reste) }\end{array}$ & $\begin{array}{l}\text { Alternative C } \\
(\text { VBG) }\end{array}$ \\
\hline abs. \pm 15 & 11.346 .200 & 11.257 .362 & 10.732 .294 & 9.213 .083 & 8.377 .567 \\
abs. \pm 25 & 11.482 .529 & 11.345 .993 & 10.327 .738 & 8.715 .887 & 8.193 .397 \\
abs. \pm 40 & 11.131 .091 & 10.978 .868 & 9.797 .769 & 8.029 .045 & 7.881 .482 \\
\hline
\end{tabular}




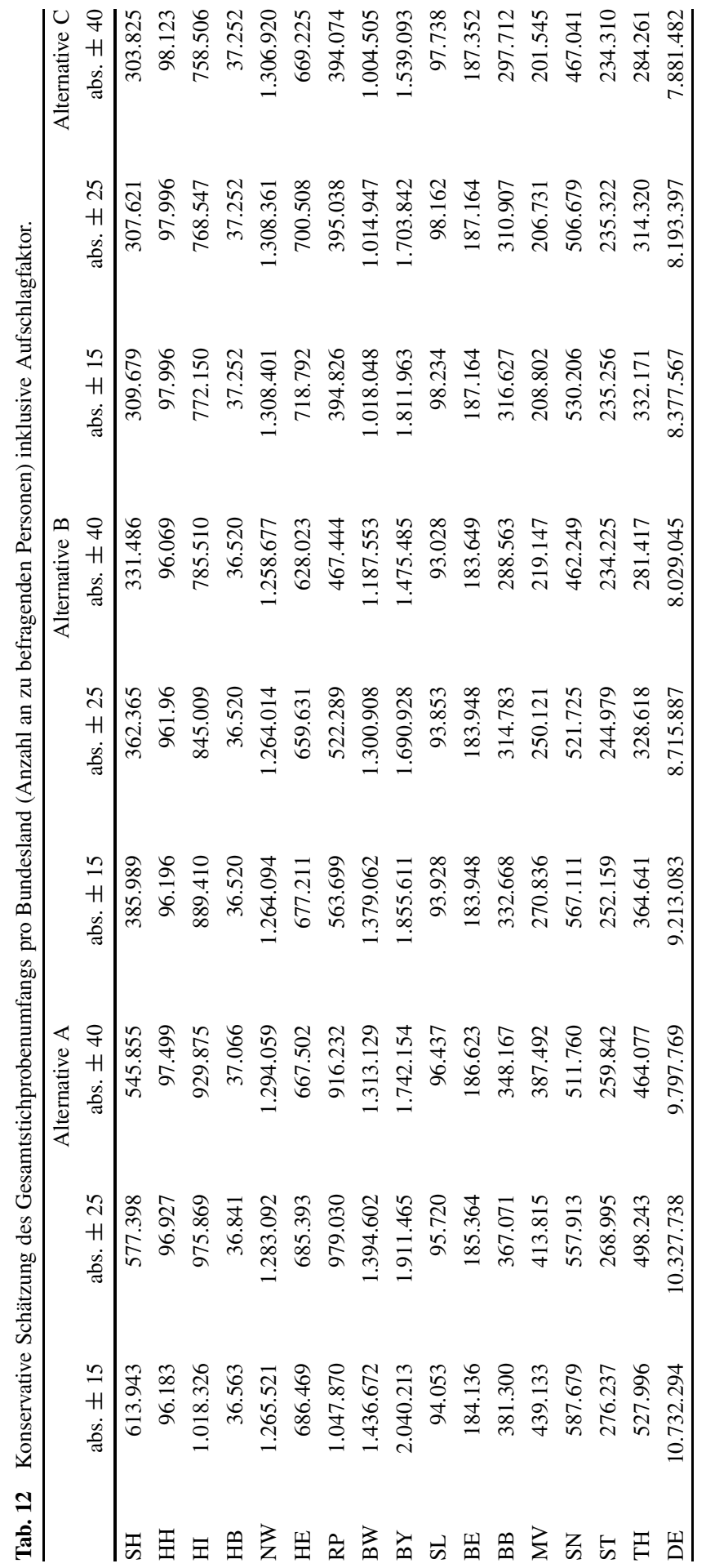




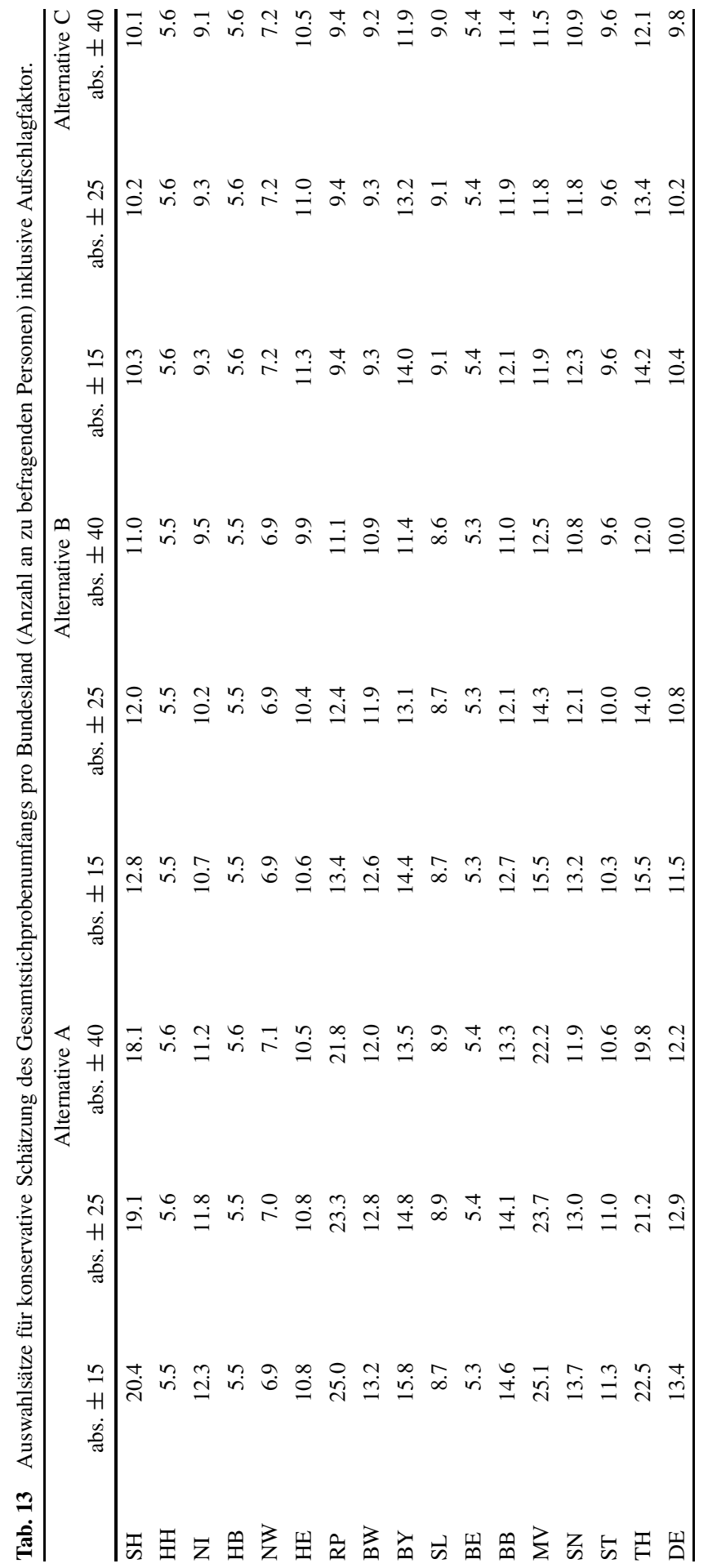




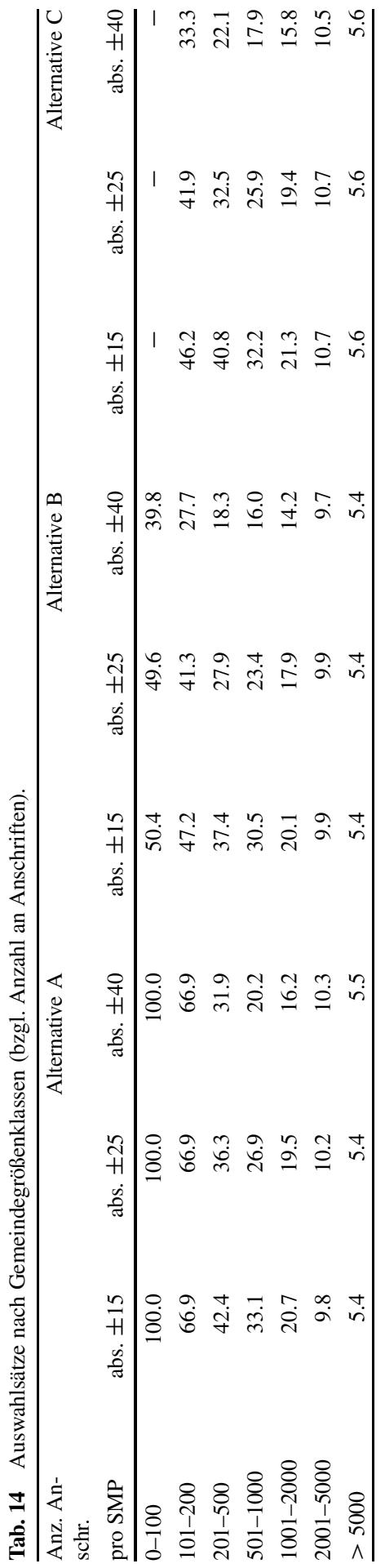


sondere bei Alternative $\mathrm{C}$ etwas besser über die verschiedenen Gemeindegrößen verteilt. Darüber hinaus gibt es im Vergleich zu Alternative A bei Alternative B und insbesondere bei Alternative C viel weniger SMPs, die den Klassen der sehr kleinen SMPs zuzuordnen sind, da viele kleine Gemeinden zu größeren SMPs zusammengefasst wurden. Im Allgemeinen ist die Wahl der Methodik (Schichtungsalternative und Präzisionszielfunktion) jedoch eine politische Entscheidung. Auch eine bundeslandspezifische Wahl der Methodik ist aus wissenschaftlicher Sicht möglich.

\section{Zusammenfassung und Ausblick}

Das Untersuchungsziel des Artikels orientiert sich, wie in Abschn. 2.3.3 beschrieben, an den Folgen des Urteils des Bundesverfassungsgerichts zum Zensus 2011 (siehe BVerfG 2018). Insbesondere galt es im Hinblick auf die Methodik des Zensus $2021 \mathrm{zu}$ untersuchen, inwiefern der Methodenwechsel an der 10.000er-Grenze geeignet ersetzt werden kann. Dabei spielen die Qualitätsmessung und der Umgang mit sogenannten Kleinstgemeinden eine zentrale Rolle.

Die in Kap. 1 beschriebenen Diskrepanzen der Zensusergebnisse 2011, die sich in Folge des Methodenwechsels für Gemeinden mit weniger und mehr als 10.000 Einwohnern ergaben, sowie die Ausführungen des Bundesverfassungsgerichtes, nach denen die Kritik an der Methodik des Zensus 2011 in geeigneter Form bei der Gesetzgebung für das Zensusgesetz 2021 berücksichtigt werden soll, bedingen eine Anpassungen der Methodik für den Zensus 2021. Diese Anpassungen sollten auf den Erfahrungen des Zensus 2011 basieren, auch wenn aufgrund des Mangels an detaillierten Informationen genaue Gründe für die offensichtlichen Diskrepanzen oberhalb und unterhalb der Grenze nicht vollständig geklärt werden konnten. Generell hatte die Festlegung der 10.000er Grenze den Vorteil, dass sich die statistische Methodik einheitlich über alle regionalen Ausweiseinheiten (SMPs) anwenden ließ. Bei einem Wegfall dieser Grenze und der direkten Folge, dass jede Gemeinde eine eigene Ausweiseinheit bildet, lässt sich die in 2011 bewährte Stichprobenziehung und Schätzmethodik inklusive der Qualitätsmessung (relativer Standardfehler von 0,5\%) auf Grund der sehr hohen Anzahl sehr kleiner Gemeinden nicht mehr statistisch adäquat umsetzen. Somit ergab sich die Frage, inwieweit entweder Vollerhebungen von Gemeinden herangezogen werden müssen oder eine geeignete Zusammenfassung von Gemeinden als regionale Ausweiseinheit definiert werden kann.

Die Ausführungen in Kap. 3.6 zeigen, dass eine vollständige Berücksichtigung sehr kleiner Gemeinden als regionale Ausweiseinheiten automatisch zu Vollerhebungsschichten, sogar zu Vollerhebungen in ca. $10 \%$ aller Gemeinden führen würde (vergleiche Tab. 9). Zwei Gründe sprechen gegen eine solche Vorgehensweise. Zum einen wurde vom Bundesverfassungsgericht ausdrücklich auf eine schonende Befragung und das Recht auf informationelle Selbstbestimmung der Bürger hingewiesen. Beides ließe sich mit Vollerhebungen kaum vereinbaren. Zum anderen führen derartige Vollerhebungen in einigen Bundesländern zu erheblich überproportionalen Auswahlsätzen (siehe Tab. 13), und als Folge in darunter fallenden sehr dünn besiedelten Gemeinden zu deutlich erhöhten Kosten. Größtenteils liegt dieser Umstand an den zwischen den Bundesländern sehr unterschiedlichen Gemeindestrukturen (verglei- 
che Tab. 1) - ein Umstand, der eine zwischen den Bundesländern unterschiedliche Vorgehensweise plausibel macht.

Konkretisiert wurde die Handhabung der Kleinstgemeinden mit den in Kap. 3.6 vorgestellten Alternativen A, B und C. Die Alternative A basiert auf der Festlegung, dass mindestens 100 Anschriften je Gemeinde gezogen werden. Im Unterschied zur Einzelbetrachtung aller Gemeinden in der Alternative A beruhen die Alternativen B und $\mathrm{C}$ auf geeigneten Zusammenfassungen von Gemeinden. In Alternative B wurden VBG-Reste gebildet, wobei alle Gemeinden mit mehr als 2.000 Einwohnern selbstständig betrachtet werden und diejenigen mit weniger als 2.000 Einwohnern innerhalb des Gemeindeverbands zusammengefasst werden. Dagegen beinhaltet die Alternative $\mathrm{C}$ eine vollständige Zusammenfassungen aller Gemeinden innerhalb eines Gemeindeverbandes. Mathematisch-statistisch lassen sich die Alternativen A, $\mathrm{B}$ und $\mathrm{C}$ so implementieren, dass sie zwischen den Bundesländern unterschiedlich eingesetzt werden können. Letztendlich ist es eine politische Entscheidung, welche Alternativen am besten zur Gemeindestruktur des jeweiligen Bundeslandes passen. Zentrale Argumente sind dabei unter anderem die Gesamtkosten der Umfrage, die Belastung der Auskunftspflichtigen und die zu erwartende Präzision der Schätzung. Ebenso gilt es die konkreten Gemeindegrößen im Auge zu halten, so dass möglichst weder große Gemeinden in Verbandsgemeinden zusammengefasst werden noch zu kleine Verbandsgemeindereste entstehen, in denen die technische Umsetzung einer Stichprobenziehung sich problematisch gestalten könnte.

Neben der Betrachtung des Stichprobendesigns und der Schätzmethodik wurden zwei tiefer gehende Aspekte im Rahmen dieses Artikels diskutiert, jedoch nicht final gelöst. Zum einen haben ausführliche Untersuchungen gezeigt, dass insbesondere auf Grund der hohen Zahl kleiner Gemeinden die Schichteinteilung nach Anschriftengrößen eine größere Rolle spielt als im Zensus 2011. Erste Ansätze mit einer variablen Schichtung erwiesen sich als recht effizient, sodass in den Untersuchungen dieses Artikels eine variable Schichtung mit einer Anzahl von einer bis 16 Schichten pro regionaler Ausweiseinheit verwendet wurde. Generell stellt sich jedoch die Fragen, inwieweit die Anwendung einer optimalen Schichteinteilung die Effizienz des Stichprobendesign weiter vergrößern könnte. Diese Fragestellung bedarf weiterer Forschung. Zum anderen bedarf es einer Anpassung der Schätzmethodik, um über die Schätzung der Einwohnerzahl auf Verbandsgemeinden oder Verbandsgemeinderesten hinaus auch kohärente Angaben für jede Gemeinde angegeben zu können. Hierbei erwiesen sich erste Versuche auf Basis einer verallgemeinerten Kalibrierung als erfolgversprechend.

Aus der Problematik der hohen Anzahl an Nullanschriften in Zensus 2011 (siehe Kap. 1), deren Auswirkung auf die Methodik gesondert untersucht wurden (siehe Münnich et al. 2013a), lassen sich zwei Empfehlungen für den Zensus 2021 ableiten. Zum einen ist es sinnvoll, die Zeitpunkte zwischen Einteilung im Zensus, Registerabzug und schließlich der tatsächlichen Durchführung des Zensus zu minimieren. Zum anderen führen spezifische Festlegungen im Zensusgesetz zu einer Einschränkung notwendiger Anpassungen in der Durchführung.

Abschließend sei nochmals auf die Worte von Präsident Voßkuhle während der Verlesung des Urteils zum Zensus beim Bundesverfassungsgericht hingewiesen. Ein Zensus sollte sich stets auf Basis der Erfahrung, die aus seiner Durchführung ge- 
wonnen werden kann, weiterentwickeln. Dazu liefern Hilfsmerkmale und Paradaten erhebliche Erkenntnisse und sollten daher für wissenschaftliche Zwecke verwendbar sein. Insbesondere im Hinblick auf einen angedachten registerbasierten Zensus 2031 sind sehr gute Kenntnisse über den aktuellen Zustand der Register sowie über mögliche Verbesserungsmöglichkeiten unabdingbar.

Danksagung Die Forschungsarbeiten zu diesem Artikel wurden im Rahmen des Forschungsprojektes Research Innovation for Official and Survey Statistics (RIFOSS) durchgeführt, das vom Statistischen Bundesamt gefördert wird. Wir danken den Kolleginnen und Kollegen, die in umfangreichen Diskussionen nach Vorträgen zur Weiterentwicklung dieses Artikels beigetragen haben. Ferner danken wir den Herausgebern, zwei anonymen Gutachtern sowie Herrn Professor Dr. Hans Kiesl für zahlreiche Kommentare, die die Präzision und Lesbarkeit des Artikels deutlich verbessert haben.

Open Access Dieser Artikel wird unter der Creative Commons Namensnennung 4.0 International Lizenz (http://creativecommons.org/licenses/by/4.0/deed.de) veröffentlicht, welche die Nutzung, Vervielfältigung, Bearbeitung, Verbreitung und Wiedergabe in jeglichem Medium und Format erlaubt, sofern Sie den/die ursprünglichen Autor(en) und die Quelle ordnungsgemäß nennen, einen Link zur Creative Commons Lizenz beifügen und angeben, ob Änderungen vorgenommen wurden.

\section{Anhang}

\section{Varianzreduktion}

In diesem Abschnitt wird der Varianzreduktionsfaktor $\left(1-\vartheta^{2}\right)$ definiert in Abschn. 2.2.1 und Formel (6) hergeleitet und bewiesen. Zur Vereinfachung definiert man zunächst

$$
\begin{aligned}
& \Theta_{x}=\sum_{h=1}^{H} \gamma_{h}^{2} \frac{1-f_{h}}{n_{h}} S_{x h}^{2}, \\
& \Theta_{y}=\sum_{h=1}^{H} \gamma_{h}^{2} \frac{1-f_{h}}{n_{h}} S_{y h}^{2} \text { und } \\
& \Theta_{y x}=\sum_{h=1}^{H} \gamma_{h}^{2} \frac{1-f_{h}}{n_{h}} S_{y x h} .
\end{aligned}
$$

Somit lässt sich die Varianz des kombinierten Regressionsschätzers definiert in (2) durch

$$
\mathrm{V}\left(\widehat{\tau}_{\mathrm{kR}}\right)=N^{2} \cdot\left(\Theta_{y}-2 \cdot \beta \cdot \Theta_{y x}+\beta^{2} \cdot \Theta_{x}\right)
$$

und ein varianzminimaler Regressionskoeffizient $\beta$ definiert in (3) durch

$$
\beta=\frac{\Theta_{y x}}{\Theta_{x}}
$$


darstellen. Durch Einsetzen der Formel für den Regressionskoeffizienten (19) in Formel (18) ergibt sich

$$
\begin{aligned}
\mathrm{V}\left(\widehat{\tau}_{\mathrm{kR}}\right) & =N^{2} \cdot\left(\Theta_{y}-2 \cdot \frac{\Theta_{y x}}{\Theta_{x}} \cdot \Theta_{y x}+\left(\frac{\Theta_{y x}}{\Theta_{x}}\right)^{2} \cdot \Theta_{x}\right) \\
= & N^{2} \cdot\left(\Theta_{y}-\frac{\Theta_{y x}^{2}}{\Theta_{x}}\right) .
\end{aligned}
$$

Dies lässt sich durch Erweitern umschreiben zu

$$
\begin{gathered}
\mathrm{V}\left(\widehat{\tau}_{\mathrm{kR}}\right)=N^{2} \cdot\left(\frac{\Theta_{y} \cdot \Theta_{x}-\Theta_{y x}^{2}}{\Theta_{y} \cdot \Theta_{x}} \cdot \Theta_{y}\right) \\
=N^{2} \cdot\left(1-\frac{\Theta_{y x}^{2}}{\Theta_{y} \cdot \Theta_{x}}\right) \cdot \Theta_{y} .
\end{gathered}
$$

Dieses Ergebnis entspricht aber gerade der Behauptung (5) mit Varianzreduktionsfaktor (6).

\section{Zusätzliche Tabellen und Grafiken zu Kap. 3 und 4}

In diesem Kapitel werden weitere Ergebnisse präsentiert, die die Erläuterungen aus den Kap. 3 und 4 vertiefen und unterstützen. In Abschn. 3.4 wird diskutiert, dass die Varianzreduktionsfaktoren zur Schätzung der Populationskennzahlen pro Gemeinde auf Basis der Stichprobe aus dem Zensus 2011 geschätzt werden. Neben der in Kap. 4 ausführlich diskutierten Unsicherheit der Varianzreduktionsfaktoren unterscheiden sie sich pro Gemeinde sehr deutlich. Dies wird in Abb. 8 für die aus der Zensusstichprobe 2011 berechneten Werte dargestellt. In Abb. 21 wird die Verteilung der Varianzreduktionsfaktoren in der Simulationsgesamtheit in Form der gemeinde-

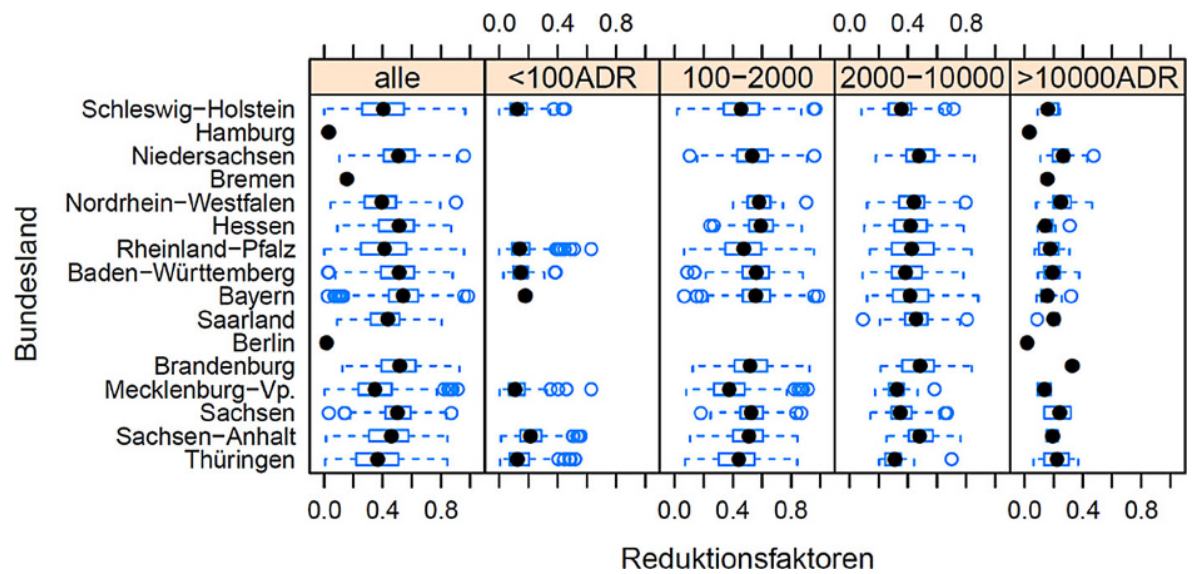

Abb. 21 Verteilung der Reduktionsfaktoren bzgl. Bundesland und Gemeindegröße (auf Basis des Simulationsdatensatzes). 
Abb. 22 Scatterplots 4 Gemeinden (synthetische Grundgesamtheit).
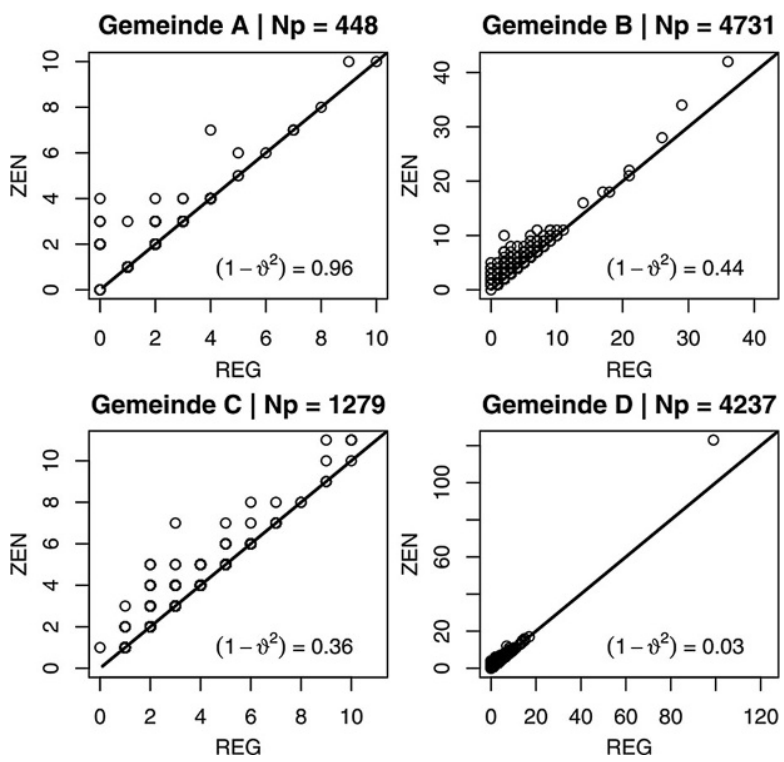

spezifischen Mittelwerte der MC-Simulation über 1.000 Wiederholungen dargestellt. Die Darstellung wird unterteilt nach Gemeindegrößen (Spalten) und Bundesländern (Zeilen). An der Größe der Boxplots wird sehr schnell deutlich, dass auch in der Simulationsgesamtheit über alle Bundesländer hinweg und insbesondere in den kleineren und mittleren Gemeinden eine starke Variation der Varianzreduktionsfaktoren zu finden ist. Zwei ähnlich große Gemeinden müssen also insbesondere nicht einen ähnlichen Varianzreduktionsfaktor haben. Darüber hinaus ist zu erkennen, dass große Gemeinden tendenziell einen geringeren Varianzreduktionsfaktor $\left(1-\varrho^{2}\right)$ haben, also die Varianz der Schätzung durch die Verwendung von Hilfsinformationen in der GREG-Schätzung mehr verringert werden kann.

In Abb. 6 wurden die Registerwerte und die Zensuswerte 2011 für drei Gemeinden auf Basis der Zensusstichprobe von 2011 miteinander verglichen. Um die Struktur der Simulationsgesamtheit zu verdeutlichen, werden in Abb. 22 die Register- und Zensuswerte auf Basis der Simulationsgesamtheit für vier ausgewählte Gemeinden miteinander verglichen. Zudem sind die Varianzreduktionsfaktoren $\left(1-\vartheta^{2}\right)$ angegeben. Zuerst ist zu erwähnen, dass die Strukturen in Abb. 22 deren in Abb. 6 ähnelt, was die Grundgesamtheit in diesem Sinne als ähnliches Abbild der Realität verifiziert. Des Weiteren wird deutlich, dass sich die Strukturen pro Gemeinde teils stark voneinander unterscheiden. Insbesondere ist die Existenz von Ausreißern in der Simulationsgesamtheit zu erwähnen, wie in der Grafik unten rechts für Gemeinde D zu erkennen.

In den Abb. 23 bis 26 werden für die vier Gemeinden, für die in Abb. 22 ein Scatterplot zu finden ist die Bootstrap-Verteilungen der Varianzreduktionsfaktoren detailliert analysiert. Wie in Abschn. 4.1 erläutert, ist der Bootstrap eine Möglichkeit zur Berechnung eines Sicherheitsaufschlagfaktors zur Abfederung der Unsicherheit der geschätzten Varianzreduktionsfaktoren. In den folgenden Abbildungen sind für 
Gemeinde A - $448 \mathrm{EW} \mid\left(1-\vartheta^{2}\right)=0.96$


Stichprobe $=3$


Abb. 23 Analyse Gemeinde A: Bootstrap-Verteilungen der Varianzreduktionsfaktoren für vier ausgewählte Stichproben auf Basis der Simulationsgesamtheit.

jede der vier Gemeinden vier Grafiken abgetragen, die jeweils eine Stichprobe der MC-Simulation repräsentieren. Innerhalb jeder Grafik ist der korrekte (über die Grundgesamtheit berechnete) Varianzreduktionsfaktor $\left(1-\vartheta^{2}\right)$ als grob gestrichelte Linie abgetragen. Der auf Basis der Stichprobe geschätzte Varianzreduktionsfaktor ist als fein-gestrichelte Linie zu erkennen. Hier wird erneut die große Unsicherheit der Schätzung deutlich, teils liegt sie nahe am korrekten Wert, teil aber auch sehr weit entfernt. Der Bootstrap-Mittelwert (aus der Bootstrap-Verteilung berechnet) ist als gepunktete Linie zu sehen. Der wie in Abschn. 4.1 vorgeschlagen mit einer Standardabweichung der Bootstrap-Verteilung korrigierte Varianzreduktionsfaktor $\left(1-\vartheta^{2}\right)$ ist als gepunktet-gestrichelte Linie dargestellt. Auch hier zeigen die Abbildungen deutlich, dass zum Teil der Aufschlagfaktor gut geeignet ist, um eine konservative Abschätzung zu erreichen.

Es gibt allerdings auch Fälle, in denen die Korrektur bei weitem nicht ausreicht beziehungsweise deutlich zu hoch ist. Alles in Allem soll durch die Abb. 23 bis 26 die Problematik der stark unterschiedlichen Struktur der Gemeinden und der starken Variation aus Stichproben geschätzten Aufschlagfaktoren verdeutlicht werden. 
Gemeinde B - $4731 \mathrm{EW} \mid\left(1-\vartheta^{2}\right)=0.44$

Stichprobe $=1$



Stichprobe $=3$



Stichprobe $=2$

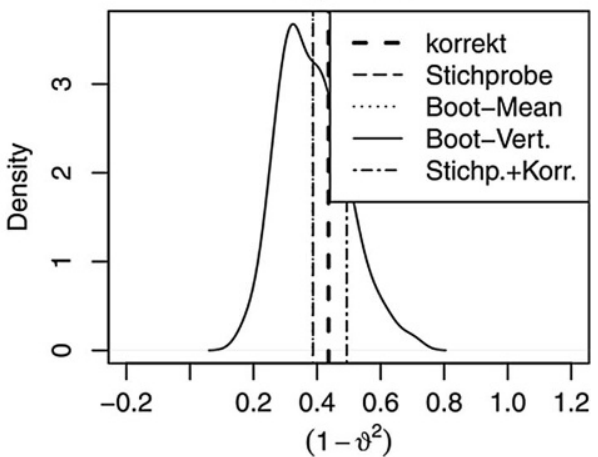

Stichprobe $=4$

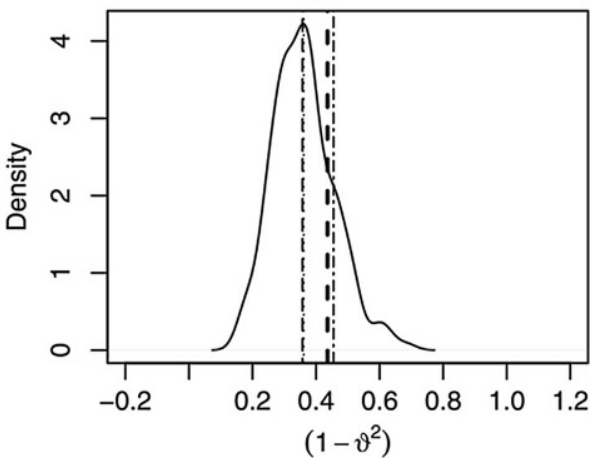

Abb. 24 Analyse Gemeinde B: Bootstrap-Verteilungen der Varianzreduktionsfaktoren für vier ausgewählte Stichproben auf Basis der Simulationsgesamtheit. 
Gemeinde C $|1279 \mathrm{EW}|\left(1-\vartheta^{2}\right)=0.36$
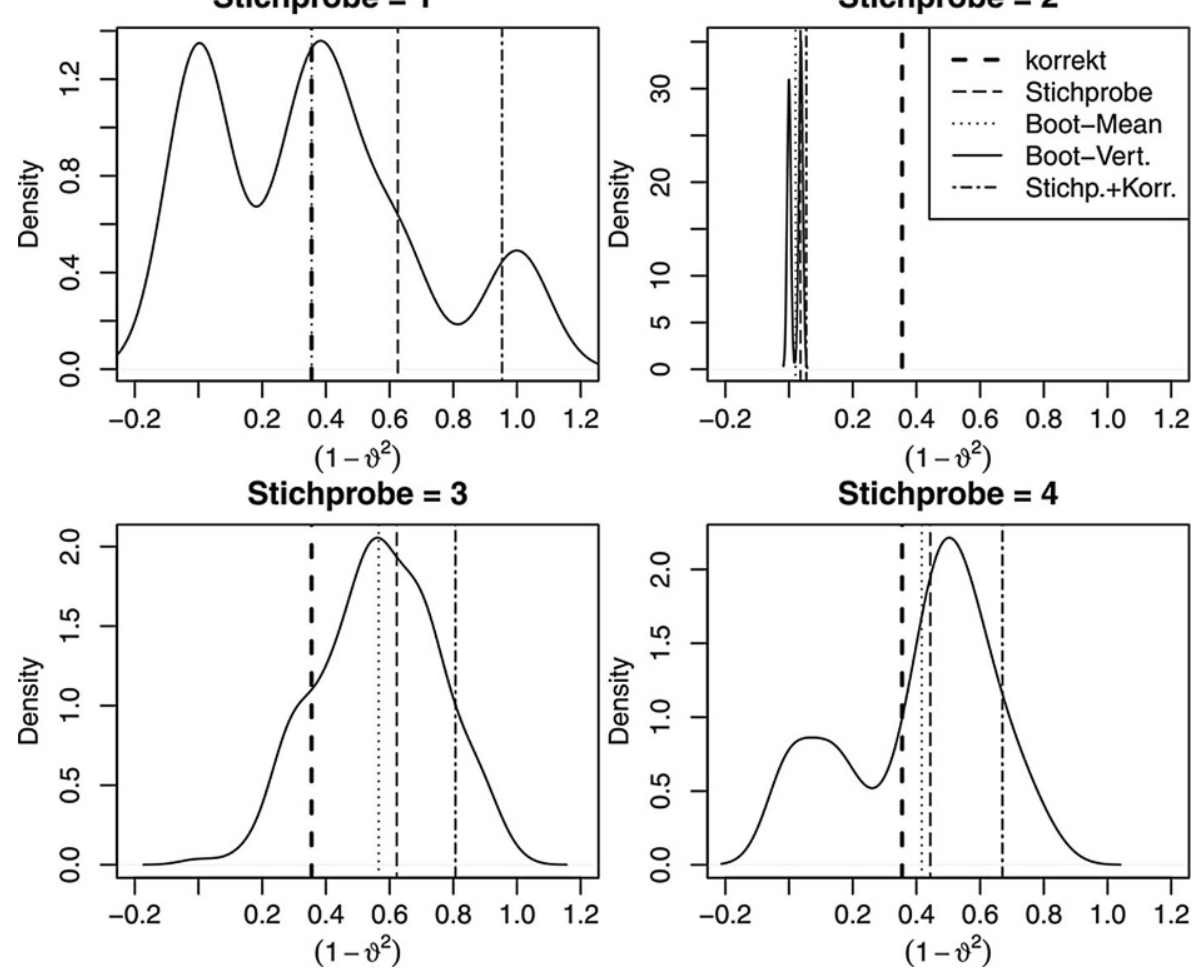

Abb. 25 Analyse Gemeinde C: Bootstrap-Verteilungen der Varianzreduktionsfaktoren für vier ausgewählte Stichproben auf Basis der Simulationsgesamtheit. 

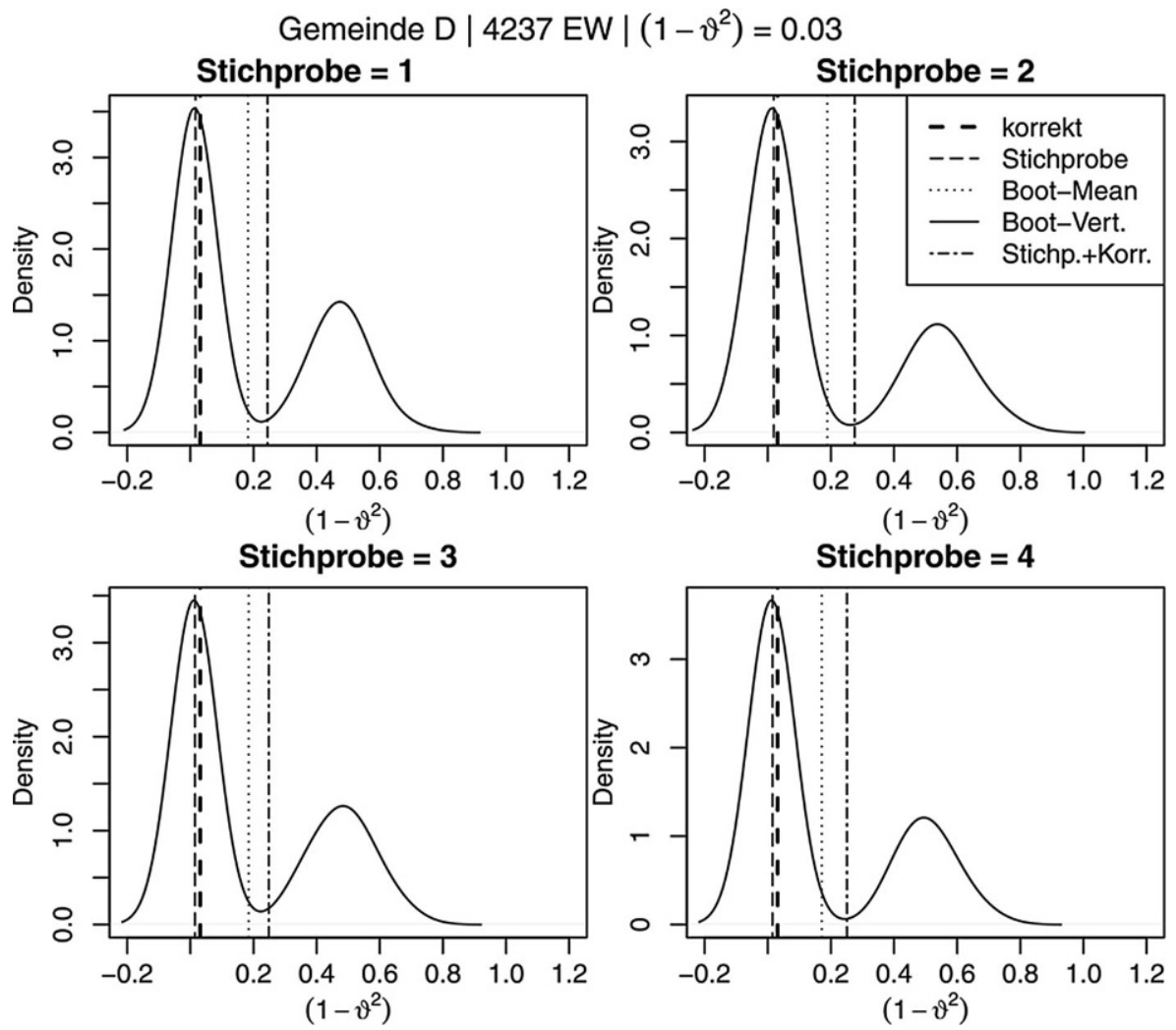

Abb. 26 Analyse Gemeinde D: Bootstrap-Verteilungen der Varianzreduktionsfaktoren für vier ausgewählte Stichproben auf Basis der Simulationsgesamtheit.

\section{Literatur}

Berg A, Bihler W (2011) Das Stichprobendesign der Haushaltsstichprobe des Zensus 2011. WISTA Wirtschaft Stat 2011(4):317-328

Burgard JP, Münnich R, Rupp M (2019) A generalized calibration approach ensuring coherent estimates with small area constraints. Research Papers in Economics, 10/19. Universität Trier. Arbeitspapier

BVerfG (2018) Urteil des Zweiten Senats vom 19. September 2018 - 2 BvF 1/15 - Rn. (1-357). ECLI:DE:BVerfG:2018:fs20180919.2bvf000115

Christensen B, Christensen S, Hoppe T, Spandel M (2015) Everything counts! AStA Wirtsch Sozialstat Arch 9(3-4):215-232

Cochran WG (1977) Sampling techniques, 3. Aufl. John Wiley \& Sons, New York

Deutscher Bundestag (2009) Zensusgesetz 2011. Gesetz über den registergestützten Zensus im Jahre 2011 vom 8. Juli 2009 (BGB1. I S. 1781). http://www.gesetze-im-internet.de/zensg_2011/. Zugegriffen: 16.09.2019

Deutscher Bundestag (2017) Zensusvorbereitungsgesetz 2021. Zensusvorbereitungsgesetz 2021 vom 3. März 2017 (BGB1. I S. 388), das durch Artikel 1 des Gesetzes vom 27. November 2018 (BGB1. I S. 2010) geändert worden ist. http://www.gesetze-im-internet.de/zensvorbg_2021/. Zugegriffen: 16.09.2019

Deutscher Bundestag (2019) Entwurf zum Zensusgesetz 2021. Entwurf eines Gesetzes zur Durchführung des Zensus im Jahr 2021. Gesetzentwurf der Bundesregierung, BR-Drucksache 19/8693 vom 25. März 2019. http://dip21.bundestag.de/dip21/btd/19/086/1908693.pdf. Zugegriffen: 16.09.2019 
European Statistical System Committee (2015) Draft regulation on statistics relating to persons and households. EEA ESSC 2015/27/7/EN

Eurostat (2013) Handbook on precision requirements and variance estimation for ESS household surveys. Eurostat Methodologies and Working Papers. KS-RA-13-029-EN-N. Eurostat, Luxemburg

Friedrich U, Münnich R, de Vries S, Wagner M (2015) Fast integer-valued algorithms for optimal allocations under constraints in stratified sampling. Computational Statistics \& Data Analysis 92:1-12

Friedrich U, Münnich R, Rupp M (2018) Multivariate optimal allocation with box-constraints. Aust J Stat 47(2):33-52

Gabler S, Ganninger M, Münnich R (2012) Optimal allocation of the sample size to strata under box constraints. Metrika 75(2):151-161

Göß1 T (2018) Der Zensus vor dem Bundesverfassungsgericht. Bayern in Zahlen 10:685-696

Kish L (1987) Statistical design for research. John Wiley \& Sons, Hoboken, New Jersey

Krug W, Nourney M, Schmidt J (2001) Wirtschafts- und Sozialstatistik: Gewinnung von Daten, 6. Aufl. Oldenburg, München

Leischner S, Weigelt S (2018) Anmerkung zu BVerfG, Urteil vom 19. September 2018, 2 bvf1/15, 2 bvf2/15. Neue Zeitschr Verwaltungsrecht 37(22):1703 (1731-1733)

Lohr S (2010) Sampling: design and analysis. Advanced series. Brooks/Cole, Boston

Münnich R, Gabler S, Ganninger M, Burgard JP, Kolb J-P (2011) Das Stichprobendesign des registergestützten Zensus 2011. Methoden - Daten - Analysen 5(1):37-61

Münnich R, Gabler S, Ganninger M, Burgard JP, Kolb J-P (2012a) Stichprobenoptimierung und Schätzung im Zensus 2011. Statistik und Wissenschaft, 21. Statistisches Bundesamt, Wiesbaden

Münnich R, Sachs E, Wagner M (2012b) Numerical solution of optimal allocation problems in stratified sampling under box constraints. Adv Stat Anal 96(3):435-450

Münnich R, Gabler S, Ganninger M, Burgard JP, Kolb J-P (2013a) Validierungsprojekt zum deutschen Zensus 2011. Statistische Ämter des Bundes und der Länder, Wiesbaden

Münnich R, Burgard JP, Vogt M (2013b) Small Area-Statistik: Methoden und Anwendungen. AStA Wirtsch Sozialstat Arch 6(3/4):149-191

Münnich R, Gabler S, Bruch C, Burgard JP, Enderle T, Kolb J-P, Zimmermann T (2015) Tabellenauswertungen im Zensus unter Berücksichtigung fehlender Werte. AStA Wirtsch Sozialstat Arch 9(3/4):269-304

Rao J, Molina I (2015) Small area estimation, 2. Aufl. Wiley Series in Survey Methodology. John Wiley \& Sons, Hoboken, New Jersey

Rendtel U (2015) Warum im Zensus die Ergebnisse der Stichprobenmethode keine Benachteiligung der großen Gemeinden darstellen. Eine Detektivarbeit. AStA Wirtsch Sozialstat Arch 9(3-4):233-249

Rupp M (2018) Optimization for Multivariate and Multi-domain Methods in Survey Statistics. Dissertation, Universität Trier

Särndal CE, Swensson B, Wretman J (1992) Model assisted survey sampling. Springer, New York

Schumaker L (2007) Spline functions: basic theory. Cambridge Mathematical Library. Cambridge University Press, Cambridge

Statistische Ämter des Bundes und der Länder (2004) Ergebnisse des Zensustests. Wirtsch Stat 8:813-833

Statistische Ämter des Bundes und der Länder (2015) Zensus 2011 - Methoden und Verfahren. Technical report, Statistisches Bundesamt. https://www.zensus2011.de/SharedDocs/Downloads/DE/ Publikationen/Aufsaetze_Archiv/2015_06_MethodenUndVerfahren.pdf?_blob=publicationFile\& $\mathrm{v}=6$. Zugegriffen: 16.09 .2019

Tzavidis N, Zhang L-C, Luna A, Schmid T, Rojas-Perilla N (2018) From start to finish: a framework for the production of small area official statistics. J Roy Stat Soc Ser A 181(4):927-979

Verma V (1991) Sampling methods. Training handbook. Statistical Institute for Asia and the Pacific (SIAP), Tokyo

Weisstein EW (2018) Bézier Curve. From MathWorld-A Wolfram Web Resource. http://mathworld. wolfram.com/BezierCurve.html. Zugegriffen: 16.09.2019

Hinweis des Verlags Der Verlag bleibt in Hinblick auf geografische Zuordnungen und Gebietsbezeichnungen in veröffentlichten Karten und Institutsadressen neutral. 